\title{
On the complex interplay between spectral harmonicity and different types of cross frequency couplings in non linear oscillators and biologically plausible neural network models
}

\author{
Damián Dellavale ${ }^{1, *}$, Osvaldo Matías Velarde ${ }^{1,}$, Germán Mato, Eugenio \\ Urdapilleta* \\ Centro Atómico Bariloche and Instituto Balseiro, Comisión Nacional de Energía Atómica \\ (CNEA), Consejo Nacional de Investigaciones Científicas y Técnicas (CONICET), \\ Universidad Nacional de Cuyo (UNCUYO), Av. E. Bustillo 9500, R8402AGP San Carlos \\ de Bariloche, Río Negro, Argentina.
}

\begin{abstract}
Background:

Cross-frequency coupling (CFC) refers to the non linear interaction between oscillations in different frequency bands, and it is a rather ubiquitous phenomenon that has been observed in a variety of physical and biophysical systems. In particular, the coupling between the phase of slow oscillations and the amplitude of fast oscillations, referred as phase-amplitude coupling (PAC), has been intensively explored in the brain activity recorded from animals and humans. However, the interpretation of these CFC patterns remains challenging since harmonic spectral correlations characterizing non sinusoidal oscillatory dynamics can act as a confounding factor.

Methods:

Specialized signal processing techniques are proposed to address the complex interplay between spectral harmonicity and different types of CFC, not restricted only to PAC. For this, we provide an in-depth characterization of the Time Locked Index (TLI) as a novel tool aimed to efficiently quantify the harmonic content of noisy time series. It is shown that the proposed TLI measure is more robust and outperform traditional phase coherence metrics (e.g. Phase Locking Value, Pairwise Phase Consistency) in several aspects.

Results:

We found that a non linear oscillator under the effect of additive noise can produce spurious CFC with low spectral harmonic content. On the other hand, two coupled oscillatory dynamics with independent fundamental frequencies can
\end{abstract}

\footnotetext{
${ }^{*}$ Corresponding authors.

Email addresses: dellavale@cab.cnea.gov.ar (Damián Dellavale), urdapile@gmail.com (Eugenio Urdapilleta)

${ }^{1}$ Equal contribution.
} 
produce true CFC with high spectral harmonic content via a rectification mechanism or other post-interaction nonlinear processing mechanisms. These results reveal a complex interplay between CFC and harmonicity emerging in the dynamics of biologically plausible neural network models and more generic non linear and parametric oscillators.

Conclusions:

We show that, contrary to what is usually assumed in the literature, the high harmonic content observed in non sinusoidal oscillatory dynamics, is neither sufficient nor necessary condition to interpret the associated CFC patterns as epiphenomenal. There is mounting evidence suggesting that the combination of multimodal recordings, specialized signal processing techniques and theoretical modeling is becoming a required step to completely understand CFC patterns observed in oscillatory rich dynamics of physical and biophysical systems.

Keywords: Non linear oscillators, Biologically plausible neural network models, Cross frequency couplings, Time Locked Index, Instantaneous frequency estimation, Systems Neuroscience

1 Highlights

- Time locked index efficiently quantifies the harmonic content of noisy time series.

- A non linear oscillator under the effect of additive noise can produce spurious cross frequency couplings (CFC) with low spectral harmonic content.

- Two coupled oscillatory dynamics with independent fundamental frequencies can produce true $\mathrm{CFC}$ with high spectral harmonic content via rectification mechanisms or other post-interaction nonlinear processing mechanisms.

- A non sinusoidal oscillatory dynamics with high harmonic content is neither sufficient nor necessary condition for spurious CFC.

- A complex interplay between CFC and harmonicity emerges from the dynamics of nonlinear, parametric and biologically plausible oscillators.

\section{INTRODUCTION}

One of the most challenging and active topics in signal processing research refers to tackling the inverse problem associated to infer the underlying mechanisms producing the time series observed from a given physical system. This is particularly true in electrophysiologically based Systems Neuroscience, in which a long standing goal is to infer the underlying multidimensional neural dynamics from spatially sparse low dimensional recordings [1. Cross frequency coupling (CFC) is a signature observed at the signal level informative on the mechanisms underlying the oscillatory dynamics. From the signal processing point of view, a 
CFC pattern emerges when certain characteristics (e.g. amplitude, phase) of a frequency band interact with others in a different band, either in the same signal or in another related one. CFC is a rather ubiquitous phenomenon observed in the oscillatory dynamics of a variety of physical and biophysical systems (see 1 ] and references therein). In particular, the phase-amplitude cross frequency coupling (PAC) observed in the electrical oscillatory activity of animal and human brains, has been proposed to be functionally involved in neuronal communication, memory formation and learning. This has motivated the development of specialized signal processing algorithms to robustly detect and quantify PAC patterns from noisy neural recordings $[2,3,4,4,6,6$, 7. However, the interpretation of these PAC patterns remains challenging due to the fact that harmonic spectral correlations characterizing non sinusoidal oscillatory dynamics can act as a confounding factor.

The concept of harmonicity refers to the degree of commensurability between the periods of the rhythms constituting the analyzed oscillatory dynamics. More precisely, two frequencies $f_{2}$ and $f_{1}$ are commensurable if they satisfy $f_{2} / f_{1} \in \mathbb{Q}$, while harmonic frequencies are related by an integer ratio, i.e. for $f_{2}>f_{1}$ they satisfy $f_{2} / f_{1} \in \mathbb{Z}$. In terms of the Fourier analysis, harmonicity can be thought as the amount of spectral power concentrated at harmonic frequencies (i.e. spectral harmonicity). In the context of CFC, harmonicity is measured as the degree of phase synchronization between rhythms pertaining to different frequency bands (i.e. PPC: phase-phase cross frequency coupling). Thus, the proposed harmonicity analysis would be worthy in many fields involving the study of physical and biophysical systems through low dimensional time series. For instance, the quantification of spectral harmonicity associated to non sinusoidal neural oscillations can serve as a measure of spatial synchronization in non invasive brain recordings like scalp electroencephalography (EEG) and magnetoencephalography (MEG) [8]. In addition, the on and off medication states of patients with Parkinson's disease can be distinguished by means of the non sinusoidal waveform shape of the exaggerated beta band oscillations observed in invasive (intracerebral EEG) and non invasive (scalp EEG) recordings 9, 10. Moreover, the harmonicity observed in intracerebral EEG recorded from epilepsy patients has been used to effectively distinguish between harmonic and non harmonic PAC patterns putatively linked to two essentially different mechanisms of seizure propagation [11. Furthermore, when two oscillatory inputs converge in a nonlinear integrator (e.g. a neuron), new harmonic and non harmonic (a.k.a. emergent) oscillations are generated via the frequency mixing mechanism [12. Importantly, oscillations emerging from this mechanism entrain unit activity [12, suggesting that frequency mixing is intrinsic to the structure of spontaneous neural activity and contributes significantly to neural dynamics. Recently, it has been reported that frequency mixing is widely expressed in a state and region-dependent manner in cortical and subcortical structures in rats 12. In this context, spectral harmonicity could be used as a surrogate of the frequency mixing mechanism and emergent components entraining unit activity, thus, our proposed method for harmonicity quantification complements the tools described in [12]. The evidence discussed above suggest that the quantification 
of harmonicity of invasive and non invasive neural recordings from humans and animal models can be used as a biomarker to characterize physiological and also pathological brain states like those observed in Parkinson's disease and epilepsy. In this work we provide an in-depth characterization of the Time Locked Index (TLI) as a novel tool aimed to efficiently quantify the harmonic content of noisy time series. In addition, the TLI is used together with other proposed signal processing techniques to quantitatively analyze the complex interplay between spectral harmonicity and different types of CFC patterns, not restricted only to PAC.

\section{METHODS}

\subsection{Synthetic and simulated dynamics}

The spectral harmonicity and CFC patterns were analyzed in a variety of synthetic and simulated oscillatory dynamics in presence of intrinsic and extrinsic additive noise. In Appendix A.1 it is described the formulation used to synthesize amplitude-modulated time series. Appendix A.2 and Appendix A.3. provide the equations for simulating the dynamics associated to the Van der Pol oscillator and a 2nd order parametric oscillator, respectively.

In what follows we define an analytically tractable model capable to produce unidirectional PAC with external drive. In this model the slow rhythm represents an external sensory input modulating the fast oscillations in sensory circuits (see discussion in [13]). The characteristics of the oscillatory dynamics and the PAC patterns elicited by the proposed biologically plausible neural network architecture have been extensively analyzed in our previous works [1, 14. In brief, the model consists of a single excitatory and a single inhibitory population that are reciprocally connected (Figure 1). This representation follows the model introduced in [15], it is a minimal version of a system capable of generating oscillations [15, 16]. The dynamics of the two populations can be written as,

$$
\left\{\begin{array}{l}
\tau_{1} \dot{m}_{1}=-m_{1}+S\left(I_{1}\right) \\
\tau_{2} \dot{m}_{2}=-m_{2}+S\left(I_{2}\right)
\end{array}\right.
$$

where $m_{i}$ and $\tau_{i}$ represent the output of the population $i \in\{1,2\}$ and the time constant, respectively. The output of this representation is constituted by the currents $I_{1}=G_{2} m_{2}\left(t-\Delta_{2}\right)+H_{1}+\eta_{1}$ and $I_{2}=G_{1} m_{1}\left(t-\Delta_{1}\right)+H_{2}+\eta_{2}$, where $G_{i}$ indicates the efficacy of the interactions, $H_{i}$ is a external input and $\Delta_{i}$ are delays in the transmission of the interaction. The terms $\eta_{i}$ represent additive white Gaussian noise (AWGN) to the inputs $I_{i}$. More precisely, $\eta_{i} \approx \mathcal{N}\left(0, \sigma_{i}\right)$. 


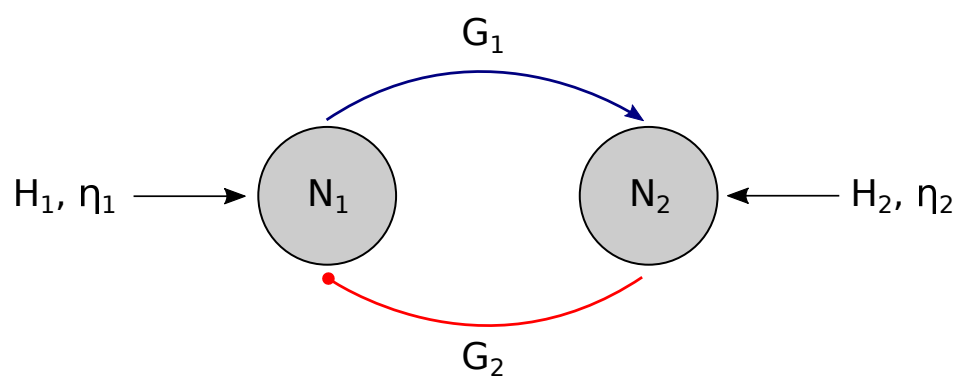

Figure 1: Biologically plausible network for unidirectional PAC with external drive representing a slow sensory input entraining fast oscillations underpinning local neural processing in a cortical oscillator (Sensory entrainment).

Regarding the instantaneous activity $A_{i}=S\left(I_{i}\right)$ of both populations, in Eqs. 1 we consider threshold linear $S\left(I_{i}\right)$ and softplus $S_{c}\left(I_{i}\right)$ transfer functions defined as,

$$
\begin{aligned}
S\left(I_{i}\right) & =\left[I_{i}\right]_{+}=\max \left(I_{i}, 0\right) \\
S_{c}\left(I_{i}\right) & =\frac{1}{c} \log \left(1+e^{c I_{i}}\right), c>0
\end{aligned}
$$

The softplus transfer function in Eq. 3 results $S_{c}\left(I_{i}\right)>0$ and converges toward the threshold linear transfer in the limit $c \rightarrow \infty$. However, these two transfer functions are essentially different regarding their order of continuity, being $S\left(I_{i}\right)$ of class $C^{0}$ (continuous but not differentiable) and $S_{c}\left(I_{i}\right)$ of class $C^{\infty}$ since it is infinitely differentiable. This has rather profound implications in the resulting dynamics. For instance, the stability of the stationary state depends on the activation function as well as of its derivative (see Eq. 4 in [1). As a consequence, $S\left(I_{i}\right)$ and $S_{c}\left(I_{i}\right)$ can produce very different stability conditions even when the latter converge to the former in the limit $c \rightarrow \infty$. A discussion on how the activation functions constituting the biologically plausible model affect the CFC patterns emerging in the resulting oscillatory dynamics is presented in Section 3.4 .

Synaptic efficacies $G_{i}$ were imposed so that the system was in the oscillatory state. The resulting oscillatory activity at $50 \mathrm{~Hz}$ belongs to the gamma band. All the parameters for network are summarized in Table 1.

Table 1: Values of the coupling parameters, time constants and delays for the model shown in Figure 1 .

\begin{tabular}{|c|c|c|c|}
\hline & $\begin{array}{c}\text { Synaptic efficacy } \\
G\end{array}$ & $\begin{array}{c}\text { Delay } \\
\Delta[\mathrm{ms}]\end{array}$ & $\begin{array}{c}\text { Time constant } \\
\tau[\mathrm{ms}]\end{array}$ \\
\hline $1 \rightarrow 2$ & 1.4 & 5 & 0.1 \\
\hline $2 \rightarrow 1$ & -1 & 5 & 0.1 \\
\hline
\end{tabular}




\subsection{Power Spectral Density}

Power spectral density (PSD) estimates were computed using the modified periodogram method with a Hann window in the time domain [17.

\subsection{Cross Frequency Coupling}

To quantify cross frequency coupling (CFC) patterns observed in the explored oscillatory dynamics, non parametric methods were used: Phase Locking Value (PLV), the Mean Vector Length (MVL) and the Modulation Index based on the Kullback-Leibler distance (KLMI) (see [6] and references therein). In the particular case of PAC, Figure 3 shows for two synthetic oscillatory dynamics the raw time series $(x(t))$ together with the band-pass filtered signals $\left(x_{L F}(t)\right.$, $\left.x_{H F}(t)\right)$, the phase of the low frequency signal $\left(\phi_{L F}(t)\right)$ and, the amplitude envelope of the high frequency signal $\left(a_{H F}(t)\right)$, as well as its phase evolution $\left(\phi_{a_{H F}}(t)\right)$ from which the PLV, MVL and KLMI metrics can be computed as follows [2, 4, 6,

$$
\begin{aligned}
P L V & =\left\langle e^{i\left(N \phi_{L F}-M \phi_{H F}\right)}\right\rangle=\frac{1}{N_{s}} \sum_{t=1}^{N_{s}} e^{i\left(N \phi_{L F}(t)-M \phi_{H F}(t)\right)}, \\
M V L & =\left\langle y_{H F} e^{i N \phi_{L F}}\right\rangle=\frac{1}{N_{s}} \sum_{t=1}^{N_{s}} y_{H F}(t) e^{i N \phi_{L F}(t)} \\
K L M I & =D_{K L}(u, p)=1+\frac{\sum_{j=1}^{N_{b}} p(j) \log p(j)}{\log N_{b}}, \\
p(j) & =\frac{\left\langle y_{H F}\right\rangle_{\phi_{L F}}(j)}{\sum_{k=1}^{N_{b}}\left\langle y_{H F}\right\rangle_{\phi_{L F}}(k)},
\end{aligned}
$$

where $t \in \mathbb{Z}$ is the discrete time index, $i$ is the imaginary unit, $N$ and $M$ are some integers, $N_{s}$ is the number of samples of the time series, $p(j)$ denotes the mean $y_{H F}(t)$ value at the $\phi_{L F}(t)$ phase bin $j\left(\left\langle y_{H F}\right\rangle_{\phi_{L F}}(j)\right)$ normalized by the sum over the bins (see histograms in Figures $3 \mathrm{C} 1, \mathrm{C} 2$ ), $N_{b}$ is the number of bins for the phase histogram and $D_{K L}$ represents the Kullback-Leibler distance between $p$ and the uniform distribution $u$. In the case of PAC (see Figure 3), Eqs. 4 to 7 are computed using $y_{H F}(t)=a_{H F}(t)$ and $\phi_{L F}(t)=\phi_{a_{H F}}(t)$. It is worth noting that PLV, MVL and KLMI metrics have been extensively used to quantify PPC and PAC, however, they can also be used to quantify other CFC types like AAC and PFC after replacing $\phi_{L F}(t), \phi_{H F}(t)$ and $y_{H F}(t)$ with the appropriate time series. A detailed discussion regarding the proper configuration and processing of the time series involved in the quantification of several CFC types including those explored in this work is given in Appendix A.4.

One of the main confounds when assessing PAC is related to the nonuniform distribution of phase angles of the modulating component $x_{L F}(t)$, which can produce spurious PAC levels [18. To detect the occurrence of this confound we computed the phase clustering (PC) as shown in Eq. 8 (see Chapter 30, p. 414 
in 17),

$$
P C_{f}=\left\langle e^{i \phi_{f}}\right\rangle=\frac{1}{N_{s}} \sum_{t=1}^{N_{s}} e^{i \phi_{f}(t)},
$$

where $\phi_{f}(t)$ is computed as described in Appendix A.4 and the subscript for the frequency band of interest is defined as $f \in\{L F, H F\}$. When $x_{L F}(t)$ has a periodic sinusoidal-like waveform shape, we obtain a rather uniform phase angle distribution $\phi_{L F}(t)$ resulting in $\left|P C_{L F}\right| \approx 0$ for a sufficiently large number of samples $N_{s}$. On the other hand, if the time series $x_{L F}(t)$ is highly non sinusoidal, we obtain a skewed distribution of phase angles producing $\left|P C_{L F}\right| \approx 1$. Worthy to note, the spurious PAC associated to high PC values can be mitigated by using narrow enough band-pass filter (BPF) to obtain the modulating low frequency oscillations $x_{L F}(t)$ (see the time series $\phi_{L F}(t)$ in Figures $3 \mathrm{C} 1, \mathrm{C} 2$ ), or by the method described in [18. In contrast, to effectively assess PAC, the BPF aimed to obtain the modulated high frequency oscillations $x_{H F}(t)$ must satisfy the restriction related to the minimum bandwidth determined by the low frequency band: $B w_{H F} \gtrsim 2 \times f_{L F}$, where $f_{L F}$ is the center frequency of the $\mathrm{BPF}$ for $x_{L F}(t)[19$.

\subsection{Time Locked Index}

A specialized tool was developed to characterize the spectral harmonicity associated to the CFC patterns observed in the explored oscillatory dynamics 11, 11. Specifically, the Time Locked Index (TLI) was implemented to efficiently quantify the presence of spectral harmonics associated to the emergence of CFC in noisy signals. The quantitative characterization of the harmonicity of the oscillatory dynamics is important given that coupled oscillatory dynamics characterized by independent frequencies or non sinusoidal repetitive waveform shapes can both elicit a similar signature in the Fourier spectrum. In particular, the traditional algorithms aimed to assess CFC based on linear filtering (e.g. PLV, MVL, KLMI) are confounded by harmonically related spectral components associated to non sinusoidal pseudoperiodic waveform shapes, reporting significant CFC levels in absence of independent frequency bands [1, 20, 21. In the TLI algorithm, time-locked averages are implemented in the time domain to exploit the phase synchronization between harmonically related spectral components constituting the non sinusoidal oscillatory dynamics. The following steps describe the procedure to compute TLI (see Figures 3B1,B2),

1. The input signal $x$ is band-pass filtered at the low (LF) and high (HF) frequency bands under analysis, producing the time series $x_{L F}$ and $x_{H F}$, respectively. Z-score normalization is applied on the time series $x_{L F}$ and $x_{H F}$ to ensure the TLI metric is independent of the signals amplitude.

2. The time instants corresponding to the maximum amplitude (or any other particular phase) of both time series, $x_{L F}$ and $x_{H F}$, are identified in each period of the low frequency band $\left(T_{L F}\right)$. These time values for the slow and fast oscillation peaks are recorded in the time vectors $t_{L F}$ (red down-pointing 
triangles in Figures $3 \mathrm{~B} 1, \mathrm{~B} 2$ ) and $t_{H F}$ (green up-pointing triangles in Figures 3B1,B2), respectively.

3. Epochs $E_{H F}^{t_{H F}}$ with a length equal to one period of the low frequency band $\left(T_{L F}\right)$ centered at the fast oscillation peaks $\left(t_{H F}\right)$ are extracted form the time series $x_{H F}$. Averaging over these epochs is computed to produce a mean epoch $\left\langle E_{H F}^{t_{H F}}\right\rangle$. Note that the latter is a time-locked averaging due to the fact that every single epoch $E_{H F}^{t_{H F}}$ is centered at the corresponding time instant $t_{H F}$.

4. Epochs $E_{H F}^{t_{L F}}$ with a length equal to one period of the low frequency band $\left(T_{L F}\right)$ centered at slow oscillation peaks $\left(t_{L F}\right)$ are extracted form the time series $x_{H F}$. Averaging over these epochs is computed to produce a mean epoch $\left\langle E_{H F}^{t_{L F}}\right\rangle$. Note that the latter is also a time-locked averaging, now with epochs centered at the corresponding time instants $t_{L F}$.

5. Finally, the TLI is computed as follows,

$$
\mathrm{TLI}=\frac{\max \left(\left\langle E_{H F}^{t_{L F}}\right\rangle\right)-\min \left(\left\langle E_{H F}^{t_{L F}}\right\rangle\right)}{\max \left(\left\langle E_{H F}^{t_{H F}}\right\rangle\right)-\min \left(\left\langle E_{H F}^{t_{H F}}\right\rangle\right)} .
$$

In the case that the time series $x$ were predominantly constituted by harmonic spectral components, the fast $\left(x_{H F}\right)$ and slow $\left(x_{L F}\right)$ oscillatory dynamics are characterized by a high degree of synchronization in time domain (i.e. phaselocking). As a consequence, the amplitude of $\left\langle E_{H F}^{t_{L F}}\right\rangle$ results comparable to that of the $\left\langle E_{H F}^{t_{H F}}\right\rangle$ and so we obtain TLI $\approx 1$ (see Figures $3 \mathrm{~A} 1, \mathrm{~B} 1$ ). On the other hand, if the spectral energy of the time series $x$ is not concentrated in narrow harmonically related frequency bands, the fast $\left(x_{H F}\right)$ and slow $\left(x_{L F}\right)$ rhythms will be not, in general, phase-locked. Therefore, the amplitude of $\left\langle E_{H F}^{t_{L F}}\right\rangle$ is averaged out to zero and TLI $\approx 0$ is obtained for a sufficiently large number of samples $N_{s}$ (see Figures $3 \mathrm{~A} 2, \mathrm{~B} 2$ ).

It is worth noting that the phase synchronization between the band-pass filtered time series $\left(x_{L F}\right.$ and $\left.x_{H F}\right)$ can be quantified using the PLV metric, however, the TLI algorithm has two significant advantages: 1 ) The computation of the TLI measure does not require to know the harmonic ratio between the frequency bands of interest. In contrast, to compute the PLV one needs to know this harmonic ratio (i.e. the values of the integers $N$ and $M$ in Eq. (4), a priori, in order to be able to evaluate the phase-phase cross frequency coupling characterizing the harmonic spectral components [3. 2) The TLI metric can be effectively computed using slightly selective BPF to obtain the HF component $x_{H F}(t)$, i.e. filters having wide bandwidths or low steepness of the transition bands. That is, by operating in the time domain the TLI reliably assesses the degree of time-locking, even in the case in which several (harmonic) spectral components are included within the bandwidth of the filter used to obtain the fast rhythm $\left(x_{H F}\right)$. This specific capability of the TLI metric is illustrated and further discussed below in connection with Figures 5, 8, 13 and 14.

Even though the TLI is a measure bounded in the range [0,1] (see Section 2.4) and independent of the processed oscillations amplitude, the absolute value of the TLI does depend on the noise level present in the processed time series and 
on the epoch length, i.e. the number of periods of the low frequency oscillation taken to implement the time-locked average involved in the TLI computation (this is further discussed below in connection with Figures 5 to 8 and B.1). As a consequence, the TLI is not a bias-free measure and this issue must be taken into account to implement a quantitative analysis of harmonicity. Fortunately, the surrogate control analysis [6] based on sample shuffled $x_{H F}(t)$ time series described in Section 2.7 below, overcome this limitation. Besides, in the case of time series corresponding to multiple channels (e.g. multi-site recordings) or trials, a commonly used method to remove the bias is to implement a Z-score normalization across channels/trials (i.e. spatial whitening) [11.

In contrast to the PLV and TLI which are biased measures [22, the pairwise phase consistency is a bias-free metric suitable for quantifying phase-phase coupling [23, 24]. However, it should be noted that the number of arithmetic operations involved in the computation of the PLV and TLI increase linearly with the number of samples $N_{s}$ (i.e. computational complexity of $O\left(N_{s}\right)$ ), while the pairwise phase consistency measure presents a significantly higher computational complexity of $O\left(N_{s}^{2}\right)$. Another measure commonly used to assess phase synchronization is the spectral coherence (see [17, Section 26.7, p. 342). Importantly, although the definition of spectral coherence includes a normalization by the total power to produce a bounded metric in the range $[0,1]$, in the expression of spectral coherence individual phase angle vectors are weighted by power values. Therefore, results from spectral coherence are likely to be influenced by strong increases or decreases in power $([17,25)$. In other words, the spectral coherence is sensitive to phase-phase and also to amplitude-amplitude and phase-amplitude correlations between the input signals. On the other hand, the TLI measure is defined as the ratio of time-locked averages computed on the same signal (HF oscillations $x_{H F}$ ), as such, it results an amplitude independent quantity only depending on the degree of synchronization between the sequence of time instants used to compute these time-locked averages $\left(t_{L F}\right.$ and $\left.t_{H F}\right)$. As a result, the TLI metric is sensitive only to PPC between the input rhythms. The source code for the computation of TLI together with test script examples implemented in Matlab ${ }^{\circledR}$ and Python are freely available at, https://github.com/damian-dellavale/Time-Locked-Index/.

We are willing to provide technical support to investigators who express an interest in implementing the TLI metric in other programming languages, integrate it in open-source software toolboxes or use it for non-profit research activities. The potential of the TLI metric to improve the characterization and aid the interpretation of PAC patterns observed in invasive neural recordings obtained from epileptic patients and in simulated dynamics of biologically plausible networks, has been demonstrated in our previous works [1, 11. In this paper we extent the harmonicity analysis to four types of CFC patterns including an indepth characterization of the TLI performance using simulated and synthetic oscillatory dynamics under controlled levels of intrinsic noise (AWGN: additive white Gaussian noise).

Figure 2 shows two essentially different CFC scenarios in terms of the spectral harmonicity, however, they are indistinguishable by traditional metrics aimed to 
assess CFC based on band-pass linear filtering (e.g. PLV, MVL, KLMI). Figure 2 A shows phase-amplitude coupling via harmonic content. In terms of telecoms engineering, the harmonic content constituting the spectrum of a quasi-periodic non sinusoidal waveform with fundamental frequency $f_{0}$ can be though as a 'carrier' given by the harmonic $N f_{0}$, being $N \in \mathbb{Z}$ the harmonic number, and 'sidebands' $(N-1) f_{0},(N+1) f_{0}$. This spectral profile is known to produce CFC patterns in the time domain (e.g, an amplitude-modulated signal). This kind of CFC patterns will be referred as 'harmonic' CFC. Figure $2 \mathrm{~B}$ shows phaseamplitude coupling in absence of phase-phase cross frequency coupling between the 'sidebands' and the 'carrier'. That is, the 'sidebands' are not harmonics of the 'carrier' (non harmonic frequencies). This kind of CFC patterns will be referred as 'non harmonic' CFC. Importantly, traditional algorithms aimed to assess CFC (e.g. PLV, MVL, KLMI) are confounded by harmonically related spectral components associated to a single (quasi)periodic non sinusoidal dynamics, reporting significant CFC levels even in absence of underlying coupled dynamics (i.e. spurious CFC). This is due to the fact that coupled oscillatory dynamics characterized by independent frequencies (i.e. true CFC) and a single non sinusoidal oscillatory dynamics (i.e. spurious CFC) produce similar signatures in the Fourier spectrum that are hardly distinguishable by using band-pass linear filtering. 
A Signal with harmonics

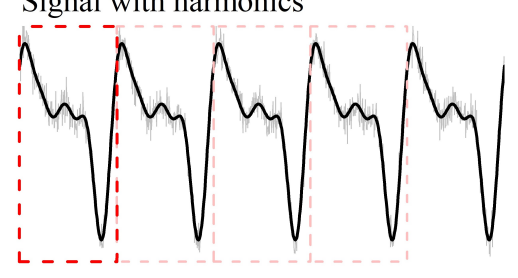

$100 \mathrm{~ms}$

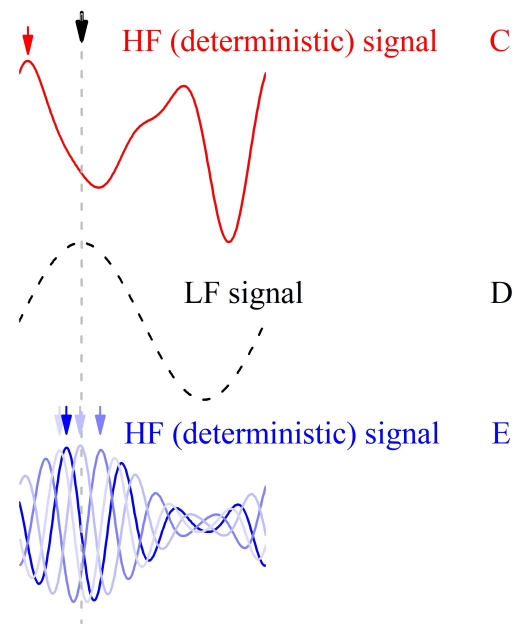

Figure 2: Coupling between low and high frequency signals. (A, C) Upper panels correspond to phase-amplitude coupling via harmonic content: A periodical nonsinusoidal signal, composed by a fundamental sinusoid at $8 \mathrm{~Hz}$ plus the first three harmonics with decreasing power, is stereotypically repeated over time (black line in panel A). By chopping the signal in consecutive segments (red boxes, one prototypical in dark red, others in light red), whose length is the period of the fundamental rhythm, the very same signal is obtained (see panel C). In panel C, the true high frequency signal, i.e. the deterministic (noiseless) signal minus the fundamental oscillatory component (thus avoiding filtering artifacts), is shown as well as the arrow corresponding to each maxima in the chopped high frequency signals (red arrow). These maxima always lie in the very same position compared to the low frequency maxima (black arrow). (D) For comparison purposes, the deterministic fundamental low frequency signal can be observed in panel D. (B, E) Lower panels correspond to a phase-amplitude modulated signal: The phase of a fundamental sinusoid at $8 \mathrm{~Hz}$ modulates the intensity of a high frequency signal at $65 \mathrm{~Hz}$. Here, high frequency signals corresponding to chopped segments (blue boxes) does not result in a single trace (see panel E). Since the modulation is developed only through amplitude, low and high frequency signals are not tightly coupled regarding phase relationships, and each maxima of the high frequency chopped signal (blue arrows) has a distribution (over phases, or relative time) with respect to the low frequency maxima (black arrow). A small level of additive white Gaussian noise (see noisy signals in gray in panels $\mathrm{A}$ and $\mathrm{B}$ ) does not change conclusions and a concomitant dispersion in the location of high frequency maxima may be observed.

To examine how the TLI metric distinguishes the harmonic CFC from the non harmonic CFC patterns, we can focus on Figure 3. Figures 3A1 and 3A2 show two synthetic signals which are constituted by two coupled oscillatory dynamics plus a small level of extrinsic additive white Gaussian noise (AWGN). Figure $3 \mathrm{~A} 1$ shows that the amplitude of the fast oscillation $x_{H F}(t)$ is modulated by the phase of the slow rhythm $x_{L F}(t)$ and these two oscillatory dynamics are also phase-locked, as evidenced by the superposition of the individual LF cycles shown at the top of the raw time series $x(t)$. A similar phase-amplitude coupling is observed between the slow and fast oscillations constituting the signal shown in Figure 3A2, however, the superposition of the individual LF cycles shows no evidence of phase-locked between the slow and fast rhythms in this 
case. To differentiate these scenarios in a quantitative manner we introduce the TLI metric which exploits the fact that, for a repetitive pattern with a fixed waveform in each cycle, harmonically related frequency bands are intrinsically linked to phase locking oscillations in time domain. The computation of the TLI metric is illustrated in Figures $3 \mathrm{~B} 1$ and $3 \mathrm{~B} 2$ for the synthetic signals shown in Figures $3 \mathrm{~A} 1$ and $3 \mathrm{~A} 2$, respectively.

In the case of the signal constituted by time-locked oscillations $x_{L F}$ and $x_{H F}$ (Figure $3 \mathrm{~B} 1$ ), we obtain similar amplitudes for the time-locked averages $\left\langle E_{H F}^{t_{L F}}\right\rangle$ and $\left\langle E_{H F}^{t_{H F}}\right\rangle$ resulting in $T L I \approx 1$. On the other hand, in the case of non timelocked oscillations $x_{L F}$ and $x_{H F}$ (Figure $3 \mathrm{~B} 2$ ), $\left\langle E_{H F}^{t_{L F}}\right\rangle$ averages out resulting in $T L I \approx 0$. Importantly, these two essentially different scenarios in terms of spectral harmonicity both present the same level of PAC as evidenced by the phase-amplitude histograms shown in Figures $3 \mathrm{C} 1$ and $3 \mathrm{C} 2$. 
bioRxiv preprint doi: https://doi.org/10.1101/2020.10.15.341800; this version posted November 1, 2020. The copyright holder for this preprint (which was not certified by peer review) is the author/funder, who has granted bioRxiv a license to display the preprint in perpetuity. It is made available under aCC-BY-NC-ND 4.0 International license.
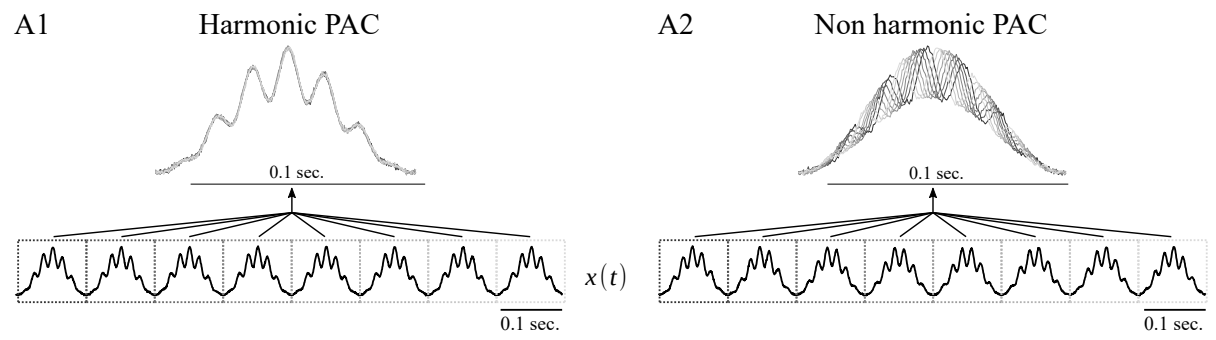

B1

B2

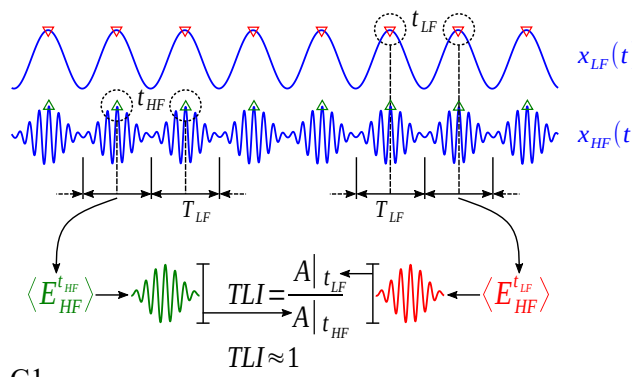

$\mathrm{C} 1$

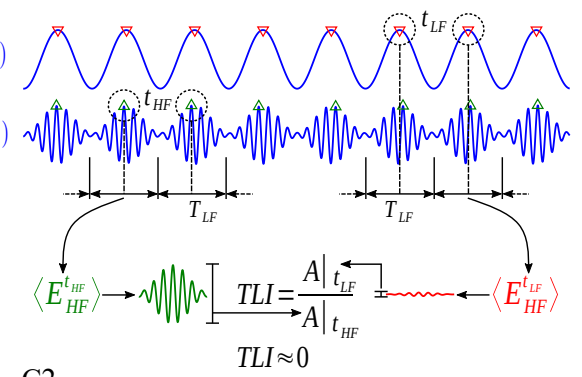

WW
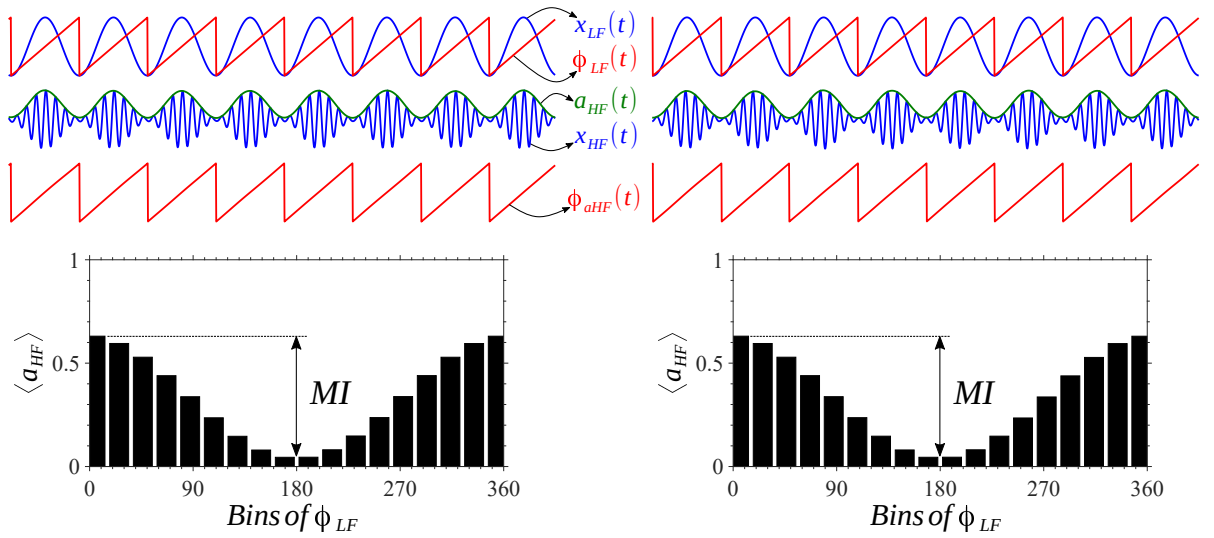

Figure 3: Synthetic amplitude-modulated signals and derived time series involved in the algorithms for quantification of PAC and harmonicity. Amplitude-modulated signals were computed as described in Section Appendix A.1 using a sinusoidal modulating at $f_{L F}=9 \mathrm{~Hz}$ and modulated oscillations at $f_{H F}=7 \times f_{L F}=63 \mathrm{~Hz}$ and $f_{H F}=7.1 \times f_{L F}=63.9 \mathrm{~Hz}$ for the harmonic PAC and non harmonic PAC, respectively. The LF signals $\left(x_{L F}(t)\right)$ were obtained by filtering the raw signal $x(t)$ using a band-pass filter centered at $9 \mathrm{~Hz}$ and a null-to-null bandwidth of $9 \mathrm{~Hz}$. The HF signals $\left(x_{H F}(t)\right)$ were obtained by filtering the raw signal $x(t)$ using a band-pass filter centered at $63 \mathrm{~Hz}$ and a null-to-null bandwidth of $81 \mathrm{~Hz}$ (see Section Appendix A.5). (A1, A2) Synthetic amplitude-modulated signals. (B1, B2) Time series used to compute the TLI metric to quantify spectral harmonicity. Note that the synthetic harmonic and non harmonic PAC patterns are characterized by $T L I \approx 1$ and $T L I \approx 0$ values, respectively. (C1, C2) Time series used to compute the PLV and KLMI metrics to quantify PAC. The histograms show the MI from which the KLMI can be computed. The histograms show the distribution of amplitude of $\mathrm{HF}$ as a function of the phase of LF. 


\subsection{Hilbert-Filter method for instantaneous frequency estimation}

Frequency-modulated patterns like phase-frequency (PFC), amplitude-frequency (AFC) and frequency-frequency (FFC) have been the least explored CFC types in neuroscience and biophysics in general, with the remarkable exception of the respiratory sinus arrhythmia associated to the PFC between the respiratory and cardiac rhythms. A possible reason for this may lie in the fact that detection methods to assess frequency-modulated patterns have been poorly described in the specialized literature 26. Importantly, the conventional CFC metrics (PLV, MVL, KLMI from Eqs. 4 to 7) in combination with equations A.22, A.23 and A.24 constitute a complete formulation to effectively assess PFC, AFC and FFC patterns, provided that a method to compute the instantaneous frequency is given. In this section we briefly discuss the conventional method used to estimate the instantaneous frequency in time and frequency domains 27, 28. In addition, we provide an alternative approach based on the Hilbert-Filter transformation of the phase time series. The proposed Hilbert-Filter transformation which operates on phase time series to produce an instantaneous frequency time series, should not be confused with the traditional Filter-Hilbert method which operates on raw time series to compute instantaneous phase and amplitude envelopes time series (see Chapter 14, p. 175 in 17]).

Let $\phi_{f}(\tau)$ be an unwrapped phase time series, corresponding to the band-limited signal $x_{f}(\tau): f \in\{L F, H F\}$, not constrained to its principal value in the inter$\operatorname{val}(-\pi, \pi]$ or $[0,2 \pi)$, i.e. $\phi_{f}(\tau)$ is a continuous function of argument $\tau \in \mathbb{R}$. We also consider that the $\phi_{f}(\tau)$ time series has been detrended through a linear fit to remove a trendline with slope $\Omega_{f}^{0}$. Then, the instantaneous frequency $\Omega_{f}(\tau)$ of the undetrended phase time series is defined as follows [27,

$$
\Omega_{f}(\tau) \triangleq \frac{d \phi_{f}(\tau)}{d \tau}+\Omega_{f}^{0}\left[\frac{\mathrm{rad} .}{\mathrm{sec} .}\right]
$$

Worthy to note, Eq. 10 implies that a bounded frequency $\Omega_{f}(\tau)$ requires a band-limited phase time series $\phi_{f}(\tau)$. This condition can be imposed by bandpass filtering $\phi_{f}(\tau)$ to restrict it to a finite frequency band of interest $(f \in$ $\{L F, H F\})$. In the discrete time domain $(t \in \mathbb{Z})$, Eq. 10 is usually approximated by a low-pass filtered version of the numerical derivative of the phase time series [28,

$$
\Omega_{f}(t) \approx h_{L P F}(t) *\left(\frac{\phi_{f}(t)-\phi_{f}(t-1)}{T_{s}}\right)+\Omega_{f}^{0},
$$

where $T_{s}=1 / f_{s}$ is the sampling time interval corresponding to the sampling rate $f_{s}$, and $*$ denotes linear convolution. Due to the fact that $\phi_{f}(t)$ is not well defined at low amplitude values of the signal $x_{f}(t)$ (see Eq. A.27), very small or large artifactual values of $\phi_{f}(t)$ sometimes occur which are amplified by the numerical derivative in Eq. 11. To mitigate these artifacts, the numerical derivative is in general smoothed by applying the low-pass filter kernel $h_{L P F}(t)$. Besides, the discrete Fourier transform $\mathcal{F}\{$.$\} of the difference equation in Eq.$ 11 can be well described by a first order approximation in the non dimensional angular frequency $\omega$, provided that the oversampling condition $\left(f_{s} \gg f: f \in\right.$ 
$\{L F, H F\}$ ) is satisfied. Under this condition, Eq. 11 can be written as (see Appendix A.6,

$$
\begin{aligned}
& \Omega_{f}(t) \approx f_{s} h_{L P F}(t) * \mathcal{F}^{-1}\left\{i \omega \Phi_{f}(\omega)\right\}+\Omega_{f}^{0} \\
& \Omega_{f}(t) \approx h_{1}(t) * \phi_{f}(t)+\Omega_{f}^{0}
\end{aligned}
$$

In Eqs. 12 and $13, \Phi_{f}(\omega)=\mathcal{F}\left\{\phi_{f}(t)\right\}$ is the discrete Fourier transform of the phase time series, $\mathcal{F}^{-1}\{$.$\} stands for the inverse discrete Fourier transform, h_{1}(t)$ is a filter with frequency response equivalent to the cascade connection of the low-pass filter $h_{L P F}(t)$ and the ideal derivator $i \omega f_{s}$. Importantly, $h_{1}(t)$ can be implemented as a high-pass or band-pass filter provided that it satisfies two main requirements: within the frequency band of interest $(f \in\{L F, H F\})$, the frequency response of $h_{1}(t)$ must approximate the magnitude response of the ideal derivator $|\omega| f_{s}$ with the following phase response,

$$
\arg \left(\mathcal{F}\left\{h_{1}(t)\right\}\right)= \begin{cases}e^{+i \frac{\pi}{2}}, & \forall \omega>0 \\ e^{-i \frac{\pi}{2}}, & \forall \omega<0\end{cases}
$$

In what follows, we shall obtain an expression equivalent to Eq. 13 by introducing the Hilbert transform with the aim to relax the requirement on the phase response of the filter $h_{1}(t)$. The Fourier representation of the Hilbert transformed phase time series is (see Chapter 11, p. 790, Eq. 11.63b in [29]),

$$
\begin{aligned}
\hat{\Phi}_{f}(\omega) & =\mathcal{F}\left\{\mathcal{H}\left\{\phi_{f}(t)\right\}\right\}=-i \operatorname{sgn}(\omega) \Phi_{f}(\omega) \\
\operatorname{sgn}(\omega) & = \begin{cases}+1, & \forall \omega>0 \\
-1, & \forall \omega<0\end{cases}
\end{aligned}
$$

From Eqs. 15 and 16, the Eq. 12 can be written as follows,

$$
\begin{aligned}
\Omega_{f}(t) & \approx f_{s} h_{L P F}(t) * \mathcal{F}^{-1}\left\{-\omega \operatorname{sgn}(\omega) \hat{\Phi}_{f}(\omega)\right\}+\Omega_{f}^{0} \\
& \approx f_{s} h_{L P F}(t) * \mathcal{F}^{-1}\left\{-|\omega| \hat{\Phi}_{f}(\omega)\right\}+\Omega_{f}^{0} \\
\Omega_{f}(t) & \approx-h_{2}(t) * \mathcal{H}\left\{\phi_{f}(t)\right\}+\Omega_{f}^{0}
\end{aligned}
$$

In this case, the frequency response of $h_{2}(t)$, within the frequency band of interest $(f \in\{L F, H F\})$, must approximate the magnitude response of the ideal derivator $|\omega| f_{s}$ with a zero-phase response (note that the constant phase of $\pi$ given by the negative sign in equation 19 can be easily introduced as an external gain of -1). As a result, the Hilbert transformed phase time series $\mathcal{H}\left\{\phi_{f}(t)\right\}$ in Eq. 19 accounts for the phase response given by Eq. 14, hence, relaxing this phase requirement on the filter $h_{2}(t)$. Besides, for offline data processing applications we can obtain a zero-phase-shift (i.e. non causal) filter by applying a magnitude mask in the frequency domain or by using a linear filter with an arbitrary phase response and reversing the phase delays. In the later case, after filtering the data in the forward direction, the filtered sequence is reversed and passed back through the filter again. Hence, we obtain zero-phase frequency response avoiding phase distortion and delays in the resulting filtered time series 
17.

As a conclusion, in the proposed method (Eq. 19p), the phase time series $\phi_{f}(t)$ is Hilbert transformed via Eq. A.26 and then passed through a zero-phase (high-pass or band-bass) filter to produce the instantaneous frequency estimation. Note that the order of the Hilbert transformation and filtering indicated in Eq. 19 can be interchanged since they are linear processes. The series of steps involved in the proposed method for the computation of the instantaneous frequency of a frequency-modulated signal (e.g. PFC) can be summarized as follows,

1. The frequency-modulated raw signal $x(t)$ is band-pass filtered around the modulated high frequency band $(H F)$ to obtain the band-limited time series $x_{H F}(t)$.

2. The Filter-Hilbert method is applied on the signal $x_{H F}(t)$ to obtain its phase time series (see Eq. A.27 and Chapter 14 in [17]).

3. Unwrap the phase time series.

4. Detrend the phase time series through a linear fit to remove a trendline with slope $\Omega_{H F}^{0}$.

5. The unwrapped and detrended phase time series is band-pass filtered around the modulating low frequency band $(L F)$ to obtain the band-limited phase time series $\phi_{H F}(t)$. Note that in the case of a frequency-modulated signal $x(t)$, the oscillatory components of $\phi_{H F}(t)$ pertain to the modulating low frequency band $L F$.

6. The Hilbert-Filter method (Eq. 19) is applied on the phase time series $\phi_{H F}(t)$ to obtain the instantaneous frequency time series $\Omega_{H F}(t)$. Note that the zero-phase filter $h_{2}(t)$ can be of type high-pass or band-pass since the main requirement is that it must approximate the magnitude of the frequency response of the ideal derivator $\left(|\omega| f_{s}\right)$ within the modulating low frequency band $L F$.

Figures $4 \mathrm{~A}$ and $4 \mathrm{D}$ show a frequency-modulated signal $x(t)$ (solid black line) and its power spectrum, respectively. The simulated dynamics was obtained from a forced 2nd order parametric oscillator (see Section 3.3). In Figure $4 \mathrm{~A}$ is possible to distinguish a PFC pattern in which a high frequency oscillation $x_{H F}(t)(H F: 10.4-190 \mathrm{~Hz}$, solid red line) is frequency-modulated by the phase of another oscillatory dynamics $x_{L F}(t)$ with lower frequency $(L F: 6.3-10.4$ $\mathrm{Hz}$, solid green line). The $x_{L F}(t)$ and $x_{H F}(t)$ time series were obtained bandpass filtering the raw signal $x(t)$ (solid black line in Figure $4 \mathrm{~A}$ ) with the filters LF BPF (dotted green line) and HF BPF (dotted red line) shown in Figure 4D, respectively. Figure $4 \mathrm{~B}$ shows the frequency time series $\Omega_{H F}(t)$ estimated using Eqs. 11 (dotted black line) and 19 (solid black line). Figure $4 \mathrm{C}$ shows the phase of the frequency time series required to assess PFC (see Eq. A.22). The lowpass filter $h_{L P F}(t)$ indicated in Eq. 11 was implemented using a moving average filter with a cutoff frequency (first sidelobe null) equal to the center frequency of the $\operatorname{HF} \operatorname{BPF}\left(f_{H F} \approx 90 \mathrm{~Hz}\right)$. The input signal was filtered in forward and reverse direction to obtain zero-phase response (no phase delays). In the time domain, this implies taking the averages over surroundings of $2 f_{s} / f_{H F}$ points, 
438 where $f_{s}=1 / T_{s}$ is the sampling rate. Figure $4 \mathrm{E}$ shows the frequency response ${ }_{439}$ of the moving average filter $h_{L P F}(t)$ (dotted black line). The solid black line ${ }_{440}$ in Figures $4 \mathrm{E}$ and $4 \mathrm{~F}$ represents the frequency response of the filter $h_{2}(t)$ used 441 to compute the Eq. 19. Note that the resulting zero-phase band-pass filter ${ }_{442}$ (LF BPF) approximate the magnitude response of the ideal derivator in the 443 modulating low frequency band ( $L F$ : $6.3-10.4 \mathrm{~Hz}$, LF BPF has central 444 frequency $f_{L F} \approx 2 \mathrm{~Hz}$ and bandwidth $\left.B w_{L F} \approx 4 \mathrm{~Hz}\right)$. The filter $h_{2}(t)$ was 445 implemented using a Tukey window in the frequency domain (see Appendix 446 A.5. 
A

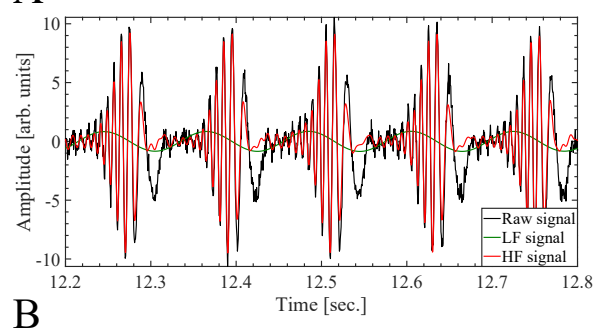

$\mathrm{B}$

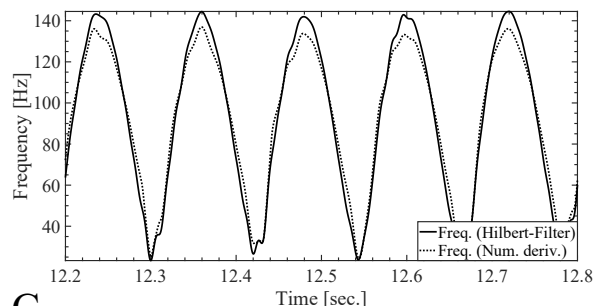

$\mathrm{C}$

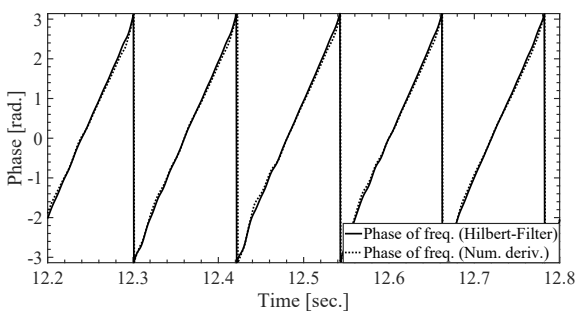

$\mathrm{D}$

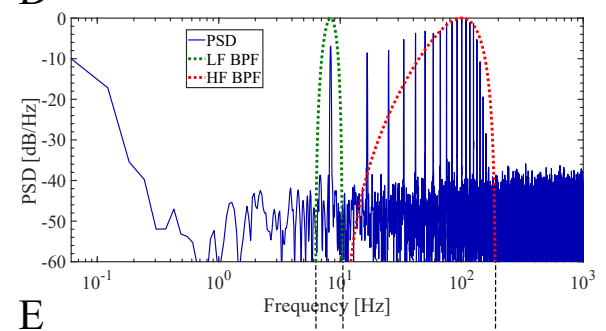

E
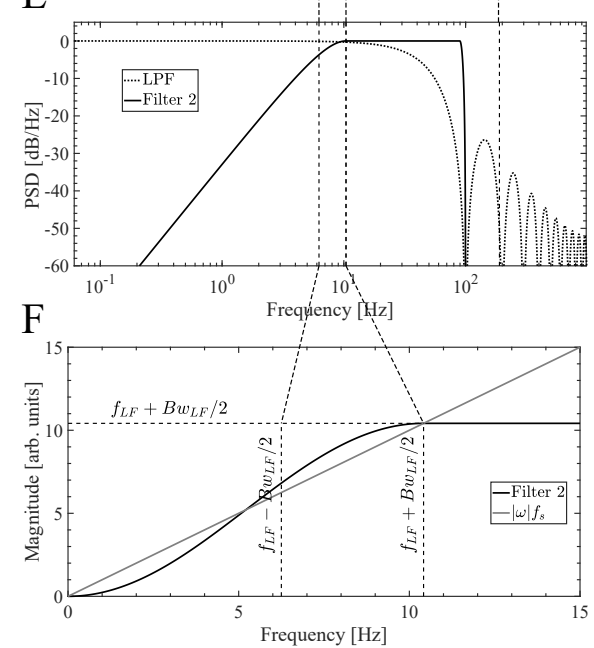

Figure 4: Hilbert-Filter method for instantaneous frequency estimation. (A) Dynamics of the parametric oscillator (solid black line) generated by simultaneously applying an off-resonance external driving $F_{e}$ and a parametric driving $W_{p}$ tuned at the same frequency $f_{e}=f_{p}=$ $f_{0} / 12 \approx 8.33 \mathrm{~Hz}$ and $\theta_{e}=0$ (see Eqs. A.16 and A.17 in Appendix A.3. The LF (solid green line) and HF (solid red line) signals where obtained band-pass filtering the raw signal (solid black line) using the BPF whose power responses (i.e. square magnitude) are shown in graph $\mathrm{D}$ as dotted green and red lines, respectively. The configuration for the parametric oscillator used in this plots is identical to that used in graphs D and E of Figure 17 (B) Instantaneous frequency time series computed for the raw signal (solid black line) shown in graph A. Solid and dotted black lines correspond to the instantaneous frequency computed using the HilbertFilter methos (Eq. 19) and the numerical derivative (Eq. 11), respectively. (C) Instantaneous phase of the frequency time series shown in graph B, computed via Hilbert transformation. (D) Power spectrum (solid blue line) of the dynamics of the parametric oscillator (solid black line in graph A). The power responses (i.e. square magnitude) of the BPF used to compute the $\mathrm{LF}$ and HF signals are shown as dotted green and red lines, respectively. (E) The solid black line represents the power response (i.e. square magnitude) of the band-pass filter $h_{2}(t)$ used to compute the instantaneous frequency time series by means of the Hilbert-Filter method (Eq. 19). The band-pass filter $h_{2}(t)$ was implemented as described in Appendix A.5 using a Tukey window in the frequency domain. The dotted black line represents the power response of the low-pass filter $h_{L P F}(t)$ used to compute the instantaneous frequency time series by means of the numerical derivative (Eq. 11). (F) Magnitude responses of the band-pass filter $h_{2}(t)$ and the ideal derivator $|\omega| f_{s}$. Regarding the oversampling condition discussed in Appendix A.6. in this case the oversampling ratio is $O S R=f_{s} / f_{L F}=2000 / 8.33 \approx 240$. 


\subsection{Harmonicity-CFC plots}

To characterize in a quantitative manner the harmonic content of CFC patterns emerging from the oscillatory dynamics explored in this work, we compute harmonicity vs. CFC plots aimed to identify correlations between these two metrics. The harmonicity (TLI) and CFC (PLV, MVL, KLMI) metrics were computed from epochs of $5 \mathrm{sec}$. or $10 \mathrm{sec}$. in length corresponding to the synthesized or simulated oscillatory dynamics (see Section 2.1) obtained for a subset of values of a parameter of interest (e.g. modulation depth, amplitude of the external driving, non linear parameter of the oscillator). In the case of simulated data, epochs of twice the required length were computed and then the first half of the time series were discarded to remove the transient period of the numerical simulation. In all the harmonicity-CFC plots shown in this work, the analyzed epochs include between 15 and 90 cycles of the slowest oscillation present in the synthetic or simulated dynamics. We verified that these results hold even in the case of using shorter epoch lengths of $\approx 7$ cycles of the slowest oscillation present in the synthetic or simulated dynamics. Then, the scatter plot between the harmonicity and CFC metrics was constructed, in which each data point corresponds to a given value of the parameter of interest. The frequency bands used to compute the harmonicity and CFC metrics were configured accordingly to the time scales of each analyzed oscillatory dynamics.

\subsection{Comodulograms and harmonicity maps}

Comodulograms for the CFC metrics (PLV, MVL, KLMI) were computed following 2, 30 and using the band-pass filters described in Appendix A.5. In all the comodulograms and harmonicity maps show in this work, the analyzed epochs include approx. 60 cycles of the slowest oscillation present in the synthetic or simulated dynamics. Each harmonicity map was constructed by computing the TLI metric for the same modulating (comodulogram $x$ axis) and modulated (comodulogram $y$ axis) frequency band combinations used to construct the corresponding CFC comodulogram. To assess the statistical significance of the CFC comodulograms, we compute a distribution of $1 \times 10^{3}$ surrogate CFC values achieved by applying the CFC measure (PLV, MVL, KLMI) to sample shuffled $\phi_{H F}(t)$ or $y_{H F}(t)$ time series (see Eqs. 4 to 7] [6]. Then, assuming a normal distribution of the surrogate $\mathrm{CFC}$ values, a significance threshold is then calculated by using $\mathrm{P}<0.001$ after Bonferroni correction for multiple comparisons 31. A similar procedure was used to assess the statistical significance of the TLI harmonicity maps using sample shuffled $x_{H F}(t)$ time series (see Section 2.4).

\subsection{Time series of CFC and harmonicity metrics}

Time series were constructed for the TLI, PLV, MVL, KLMI and PC metrics to analyze their temporal evolution during synthetic CFC patterns. The time series were constructed by computing all metrics in a sliding epoch of $20 \mathrm{sec}$. in length with $90 \%$ overlap to include several periods for the slowest modulating rhythms explored. This epoch length was an acceptable trade-off between 
statistical significance and temporal resolution capable to capture the CFC and harmonicity transients occurring in the synthetic dynamics (see the discussion about Eqs. A.1. A.2 and A.3 in Appendix A.1. Unless otherwise specified, the time series for the CFC metrics were constructed using the Algorithm 2 of Table 2 .

Table 2: Algorithms to compute the CFC time series.

\begin{tabular}{l} 
Algorithm 1 \\
\hline 1. The input time series is Z- \\
score normalized. \\
2. The whole time series is band- \\
pass filtered. \\
3. The feature (e.g. phase, \\
amplitude) is computed for \\
the whole time series.
\end{tabular}

4. The feature time series is then subdivided in sliding epochs.

5. The CFC metrics are computed for each sliding epoch.

Algorithm 2

1. The input time series is Zscore normalized.

2. The whole time series is bandpass filtered.

3. The band-pass filtered time series is then subdivided in sliding epochs.

\section{Each sliding epoch is Z- score normalized.}

\section{The feature (e.g. phase, amplitude) is computed for each sliding epoch.}

6. The CFC metrics are computed for each sliding epoch.

\section{RESULTS}

\subsection{Spectral harmonicity: Characterization of the TLI metric}

In this section we discuss the dependence of the TLI on the relevant parameters to quantify the spectral harmonicity in experimental recordings. In addition, we compare the performance of the proposed TLI metric with the conventional method to assess PPC based on the PLV measure $\left(P L V_{P P C}\right)$. For this, we compute the $P L V_{P P C}$ using Eq. 4 with the configuration given by Eq. A.19. Importantly, both harmonicity metrics are bounded in the range $[0,1]$ which is particularly convenient for the sake of comparison purposes. Due to the fact that to compute the $P L V_{P P C}$ measure using Eq. 4 one needs to know a priori the harmonic ratio between the two frequency bands of interest, i.e. the value of $M$ and $N$, the characterization presented here is based on synthetic time series in which we have precise control on these parameters. Figure 5 shows, for the case of the linear superposition of two harmonic oscillations $\left(f_{H F} / f_{L F}=7\right)$, the dependence of the TLI and $P L V_{P P C}(M=1, N=7)$ metrics on the epoch length and the bandwidth of the BPF used used to obtain 

available under aCC-BY-NC-ND 4.0 International license.

511 the fast rhythm $\left(B w_{H F}\right)$, and taking the noise level as a parameter (AWGN in 512 the range $[0 \%, 200 \%]$ of the slow oscillation amplitude). Figure $5 \mathrm{C}$ and $5 \mathrm{~F}$ show 513 the signals and power spectrum for a given set of parameter values, respectively. 
A

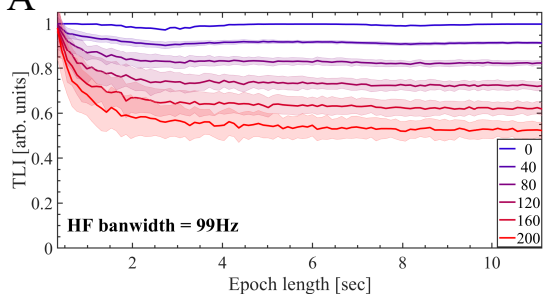

B

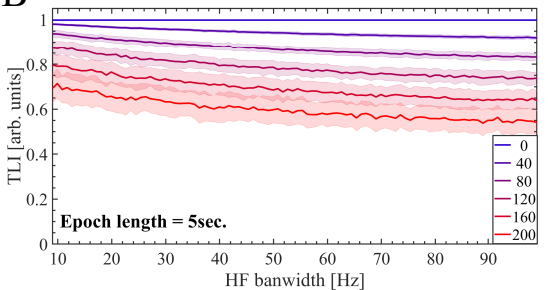

C

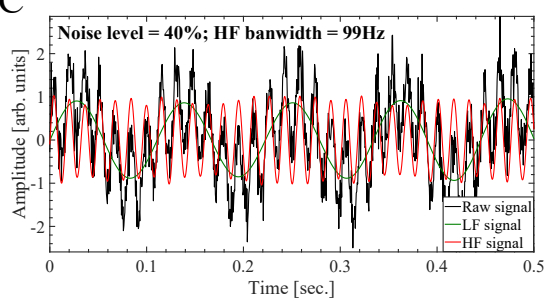

$\mathrm{D}$

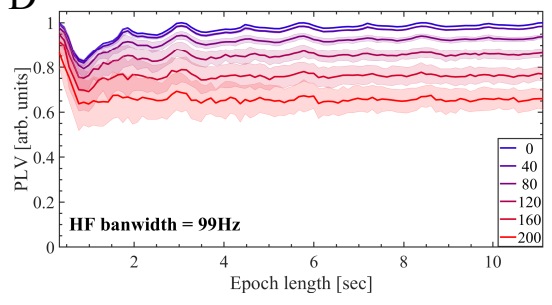

$\mathrm{E}$

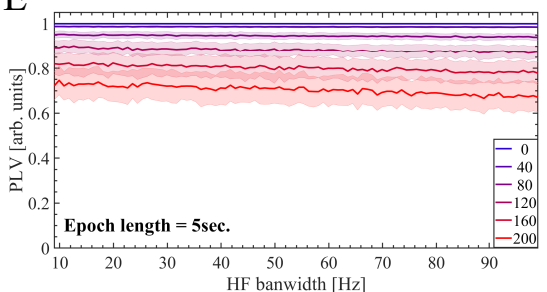

$\mathrm{F}$

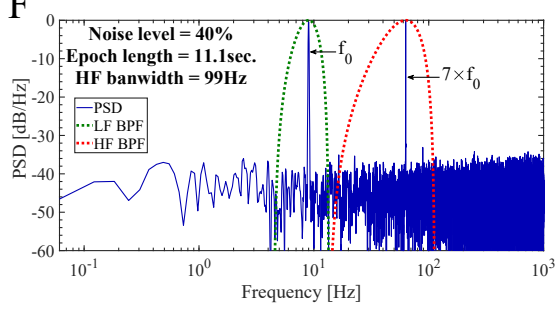

Figure 5: Performance of the TLI and $P L V_{P P C}$ in quantifying the harmonicity of a synthetic dynamics constituted by the linear superposition of two sinusoidal oscillations at $f_{0}=f_{L F}=9$ $\mathrm{Hz}$ and $f_{H F}=7 \times f_{L F}=63 \mathrm{~Hz}$. In all the cases shown in this figure, we used a sampling rate of $f_{s}=2000 \mathrm{~Hz}$ and the frequency and amplitude of the LF and HF oscillations were kept unchanged. To obtain all the band-pass filtered signals shown in this figure we use the BPF as described in Appendix A.5 The bandwidth of the BPF for the LF component (LF BPF) was kept fixed at $B w_{L F}=9 \mathrm{~Hz}$. The $P L V_{P P C}$ was computed using Eq. 4 with the configuration given by Eq. A.19 and $M=1, N=7$. (A, D) TLI and $P L V_{P P C}$ metrics as a function of the epoch length and taking the level of additive white Gaussian noise (AWGN) as a parameter. The noise level is expressed as the percent of the amplitude of the LF component at $f_{L F}=9 \mathrm{~Hz}$ scaling the standard deviation $\sigma$ of the additive white Gaussian noise $\mathcal{N}(0, \sigma)$. To compute graphs $\mathrm{A}$ and $\mathrm{D}$, the bandwidth of the $\mathrm{HF} \mathrm{BPF}$ was kept unchanged in $B w_{H F}=99 \mathrm{~Hz}$. Our implementation of the TLI algorithm (Section 2.4 requires at least 3 cycles of the low frequency oscillation $\left(f_{L F}=9 \mathrm{~Hz}\right)$, which determines the minimum epoch length shown in graphs $\mathrm{A}$ and $\mathrm{D}\left(3 / f_{L F} \approx 0.3 \mathrm{sec}\right.$.). The maximum epoch length used to compute graphs $\mathrm{A}$ and $\mathrm{D}$ was $100 / f_{L F} \approx 11.1 \mathrm{sec}$. (B, E) TLI and $P L V_{P P C}$ metrics as a function of the HF bandwidth $\left(B w_{H F}\right)$ corresponding to the BPF used to obtain the HF signal $\left(x_{H F}(t)\right)$, and taking the level AWGN as a parameter. The minimum and maximum $B w_{H F}$ values used to compute the graphs $\mathrm{B}$ and $\mathrm{E}$ were $9 \mathrm{~Hz}$ and $99 \mathrm{~Hz}$, respectively. To compute the graphs $\mathrm{B}$ and $\mathrm{E}$, the epoch length was kept unchanged in $45 / f_{L F} \approx 5$ sec. In the panels $\mathrm{A}, \mathrm{B}, \mathrm{D}$ and $\mathrm{E}$, the solid lines represent the mean values and the shaded error bars correspond to the standard deviation of 100 realizations at each point. (C) Synthetic dynamics (solid black line) together with the HF and LF signals shown as solid red and green lines, respectively. The synthetic dynamics includes additive white Gaussian noise $\mathcal{N}(0, \sigma)$ with the standard deviation $\sigma$ corresponding to the $40 \%$ of the amplitude of the $L F$ component at $f_{L F}=9 \mathrm{~Hz}$. The LF and HF signals where obtained by filtering the raw signal with the band-pass filters whose power responses are shown as dotted green $\left(B w_{L F}=9 \mathrm{~Hz}\right)$ and $\operatorname{red}\left(B w_{H F}=99 \mathrm{~Hz}\right)$ lines in graph F, respectively. (F) Power spectrum (solid blue line) of the synthetic dynamics (solid black line in graph $\mathrm{C}$ ) computed using an epoch length of $100 / f_{L F} \approx 11.1 \mathrm{sec}$. The power responses (i.e. square magnitude) of the2 $\mathrm{BPF}$ used to compute the LF and HF signals are shown as dotted green and red lines, respectively. 
Our implementation of the TLI algorithm (Section 2.4) requires at least 3 cycles of the low frequency oscillation $\left(x_{L F}(t)\right)$, which determines the minimum epoch length used to compute Figure $5\left(3 / f_{L F} \approx 0.33 \mathrm{sec}\right.$. $)$. As expected, Figures $5 \mathrm{~B}$ and $5 \mathrm{E}$ show that in presence of harmonic oscillations, the value of the harmonicity metrics decay as the AWGN level is increased ( $T L I=1$ without noise and $T L I \approx 0.5$ for a AWGN level equal to $200 \%$ of the slow oscillation amplitude). On the other hand, Figures $5 \mathrm{~A}$ and $5 \mathrm{D}$ show that in case of epoch length including sufficiently large number of slow oscillation cycles, the value of the harmonicity metrics converges to a constant value which depends on the noise level. Importantly, it was found that for short epoch length, comprising less than $\approx 10$ cycles of the slow oscillatory component, the TLI and $P L V_{P P C}$ metrics present a significant bias. This bias produces the high values $(\approx 1)$ of the harmonicity metrics in Figures $5 \mathrm{~A}$ and $5 \mathrm{D}$ for epoch length less than $\approx 1$ sec. The bias of the TLI and $P L V_{P P C}$ metrics was also investigated in presence of non harmonic oscillations. This analysis is discussed in Appendix B.1 and the obtained results support the conclusion drawn from Figures $5 \mathrm{~A}$ and $5 \mathrm{D}$.

Figures 6A and 7.A show the $P L V_{P P C}$ and TLI metrics as a function of the frequency ratio of the two oscillations constituting the synthetic dynamics, and taking the noise level as a parameter. The frequency ratio $f_{H F} / f_{L F}$ was explored for a slow oscillation with $f_{L F}=3 \mathrm{~Hz}$ pertaining the High-Delta band $(1-4 \mathrm{~Hz})$ and the fast rhythm with $f_{L F}$ ranging from the Theta band $(4-8$ $\mathrm{Hz})$ to beyond the HFO (High Frequency Oscillations) band $(100-500 \mathrm{~Hz})$. Note that this cover the conventional frequency bands for the human brain activity which have been defined on the basis of certain cognitive significance and neurobiological mechanisms of brain oscillations [17. Figures 6A and $7 \mathrm{~A}$ were computed using epochs of $5 \mathrm{sec}$. in length and BPF with constant bandwidths $\left(B w_{H F}=B w_{L F}=3 \mathrm{~Hz}\right)$, and show that both harmonicity metrics present more dispersion and lower values compared to unity indicating a detriment of their performance for increasing values of AWGN level and frequency ratio between the harmonic oscillations. Besides, we found that the small drop of the TLI metric for high frequency ratios in the case without noise shown in Figure 77A, was due to the effect of the finite sampling rate of the processed time series. In this case the oversampling rate was $f_{s} /\left(180 f_{L F}\right)=3.7$, where $f_{s}=2000 \mathrm{~Hz}$ is the sampling rate and $180 f_{L F}=540 \mathrm{~Hz}$ is the maximum frequency explored in Figure 74. We investigate this finite sampling rate effect on the TLI and $P L V_{P P C}$ metrics. In the case of the TLI measure this effect diminished exponentially with the oversampling ratio, producing a drop of the TLI value less than $\approx 5 \%$ for oversampling ratios above $\approx 5$ (data not shown). The behavior of the $P L V_{P P C}$ and TLI metrics in between the harmonic frequency ratios is shown in Figures 6B, E, H and 7B, C, D. Figures 6C, F, I and 6D, G, J show the PSD and time series for three representative cases within the explored range of frequency ratios. 

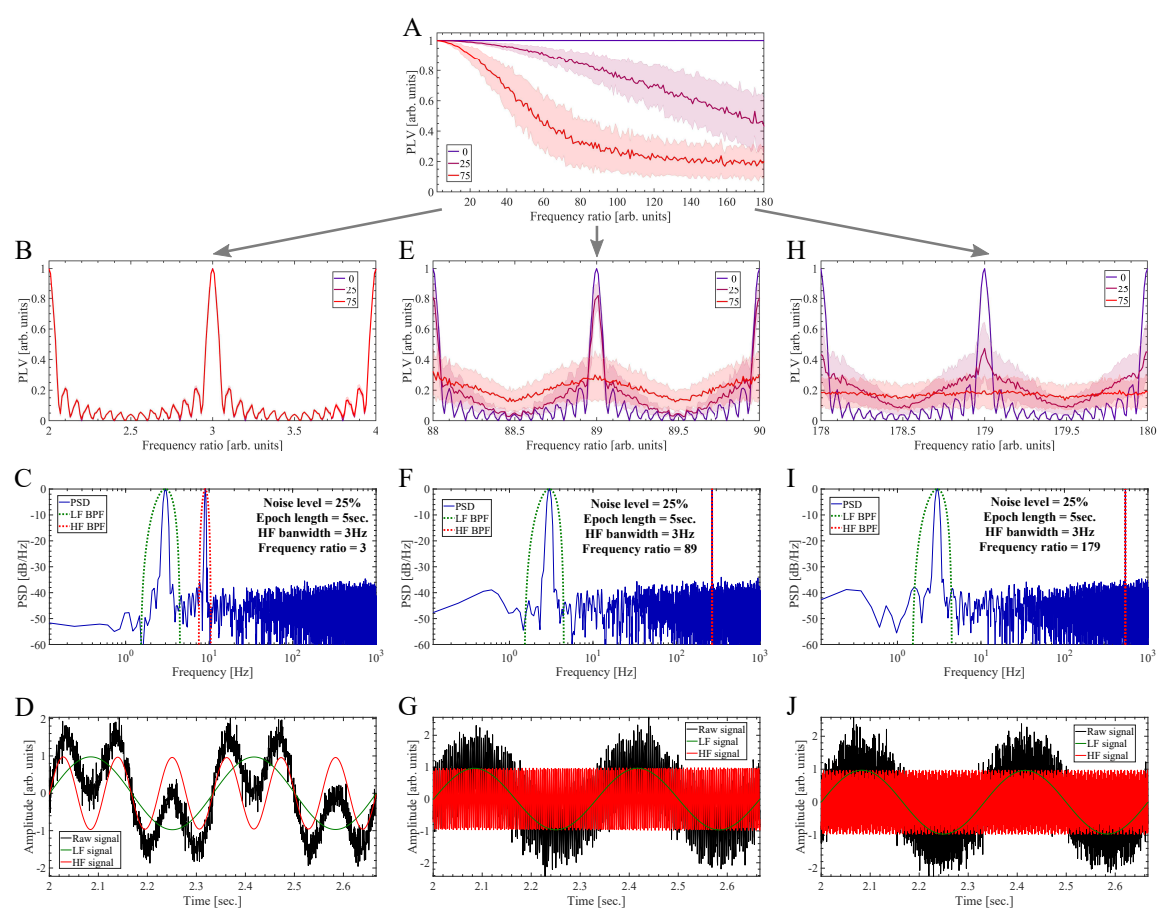

Figure 6: Performance of the $P L V_{P P C}$ in quantifying the harmonicity of a synthetic dynamics constituted by the linear superposition of two sinusoidal oscillations. In all the cases shown in this figure, we used a sampling rate of $f_{s}=2000 \mathrm{~Hz}$ and the amplitude of the LF and HF oscillations were kept unchanged. To obtain all the band-pass filtered signals shown in this figure we use the BPF as described in Appendix A.5 The bandwidth of the BPF for the LF $(\mathrm{LF} \mathrm{BPF})$ and $\mathrm{HF}(\mathrm{HF} \mathrm{BPF})$ components were kept fixed at $B w_{L F}=B w_{H F}=3 \mathrm{~Hz}$. The $P L V_{P P C}$ was computed using Eq. 4 with the configuration given by Eq. A.19 (A) $P L V_{P P C}$ intensity as a function of the frequency ratio $f_{H F} / f_{L F}$ and taking the level of additive white Gaussian noise (AWGN) as a parameter. The LF component was kept fixed at $f_{L F}=3 \mathrm{~Hz}$ and the frequency of the HF oscillation was varied in the range $2 \times f_{L F} \leq f_{H F} \geq 180 \times f_{L F}$. The noise level is expressed as the percent of the amplitude of the LF component at $f_{L F}=3 \mathrm{~Hz}$ scaling the standard deviation $\sigma$ of the additive white Gaussian noise $\mathcal{N}(0, \sigma)$. The $P L V_{P P C}$ was computed using an epoch length of $45 / f_{L F} \approx 5$ sec. (B, E, H) Evolution of the $P L V_{P P C}$ intensity in between the harmonic frequency ratios $f_{H F} / f_{L F}$ for three AWGN levels. In the panels $\mathrm{A}, \mathrm{B}, \mathrm{E}$ and $\mathrm{H}$, the solid lines represent the mean values and the shaded error bars correspond to the standard deviation of 100 realizations at each point. (C, F, I) Power spectrum (solid blue line) of the synthetic dynamics (solid black line in graphs D, G and J) corresponding to three frequency ratio values $\left(f_{H F} / f_{L F}=3,89,179\right)$. The power spectra were computed using an epoch length of $45 / f_{L F} \approx 5 \mathrm{sec}$. The power responses (i.e. square magnitude) of the BPF used to compute the LF and HF signals are shown as dotted green and red lines, respectively. (D, G, J) Synthetic dynamics (solid black line) together with the HF and LF signals shown as solid red and green lines, respectively, corresponding to three frequency ratio values $\left(f_{H F} / f_{L F}=3,89,179\right)$. The synthetic dynamics includes additive white Gaussian noise $\mathcal{N}(0, \sigma)$ with the standard deviation $\sigma$ corresponding to the $25 \%$ of the amplitude of the LF component at $f_{L F}=9 \mathrm{~Hz}$. The LF and HF signals where obtained by filtering the raw signal with the band-pass filters whose power responses are shown as dotted green $\left(B w_{L F}=3 \mathrm{~Hz}\right)$ and red $\left(B w_{H F}=3 \mathrm{~Hz}\right)$ lines in graphs $\mathrm{C}, \mathrm{F}$ and I, respectively. 


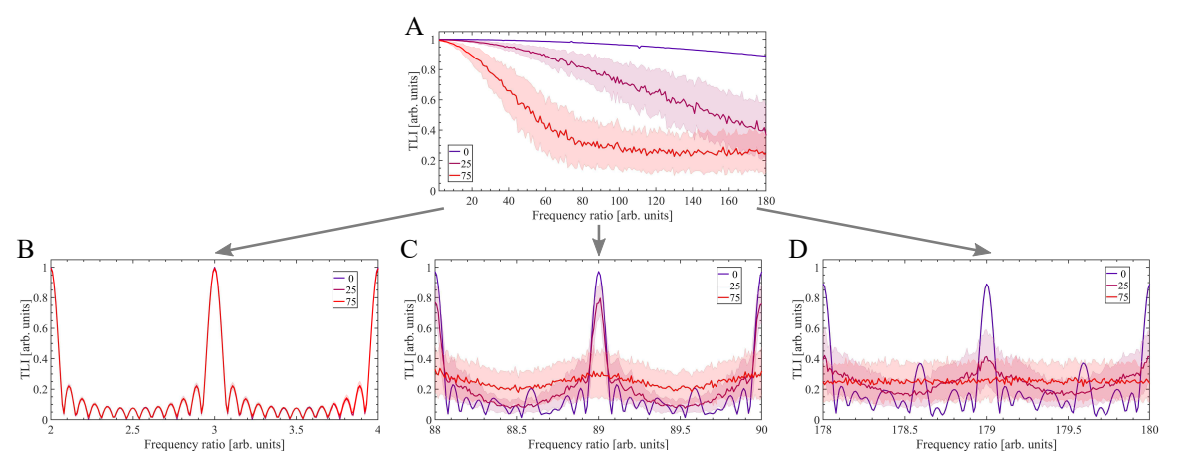

Figure 7: Performance of the TLI metric in quantifying the harmonicity of a synthetic dynamics constituted by the linear superposition of two sinusoidal oscillations. For the sake of comparison purposes this figure was computed using the same hyperparameters than those used to compute and process the synthetic dynamics shown in Figure 6

Figure 8 show the performance of the harmonicity (TLI and $P L V_{P P C}$ ) and PAC $\left(K L M I_{P A C}\right)$ metrics in a simple multi-harmonic oscillatory dynamics capable to generate PAC. For this, we compute the $K L M I_{P A C}$ using Eqs. 6 and 7 with the configuration given by Eq. A.20. The dynamics was synthesized using Eqs. A.1 A.4 and A.6 configured for the DSB-C case with a sinusoidal modulating $a(t)$ and maximum modulation depth (see the caption of Figure 8 for the complete list of parameter values). Figures $8 \mathrm{~A}$ and $8 \mathrm{C}$ for the multi-harmonic dynamics should be compared with their counterparts in the case of a single HF harmonic component shown in Figures $5 \mathrm{~A}$ and $5 \mathrm{D}$, respectively. While no significant differences are observed in the $P L V_{P P C}$ metric between these two scenarios (see Figures $8 \mathrm{C}$ and 5D), the TLI metric present higher values (close to unity) and less dispersion when multiple harmonics are included in the HF bandwidth (see Figures $8 \mathrm{~A}$ and $5 \mathrm{~A}$ ). This result is consistent with the behavior observed in Figures $8 \mathrm{~B}$ and $8 \mathrm{D}$ showing an opposite trend between the two harmonicity metrics, that is, as the HF bandwidth increases a concomitant increase in the dispersion and drop of the values occurs in the $P L V_{P P C}$ metric and the opposite is observed for the TLI measure. Figure $8 \mathrm{E}$ shows the PAC metric as a function of the epoch length and taking the AWGN as a parameter. Importantly, Figure $8 \mathrm{~F}$ shows that the $K L M I_{P A C}$ metric increases only after the HF bandwidth is wide enough to include the two sidebands $\left(6 \times f_{L F}\right.$ and $\left.8 \times f_{L F}\right)$ around the carrier $\left(f_{H F}=7 \times f_{L F}\right)$, that is $B w_{H F} \gtrsim 2 \times f_{L F}=18 \mathrm{~Hz}$. Worthy to note, the increase rate of the $K L M I_{P A C}$ curves in Figure $8 \mathrm{~F}$ is related to the steepness (i.e. transition-band width) of the BPF used to obtain the fast (amplitude-modulated) rhythm. That is, the steeper the roll-offs of the BPF the higher the increase rate of the $K L M I_{P A C}$ curves in Figure $8 \mathrm{~F}$. Note that we do not use BPF with very steep roll-offs to prevent creating artificial narrow-band oscillations [31, 32] (see Appendix A.5). Figures $8 \mathrm{G}$ and $8 \mathrm{H}$ show the PSD and time series for a representative case within the explored parameters. 

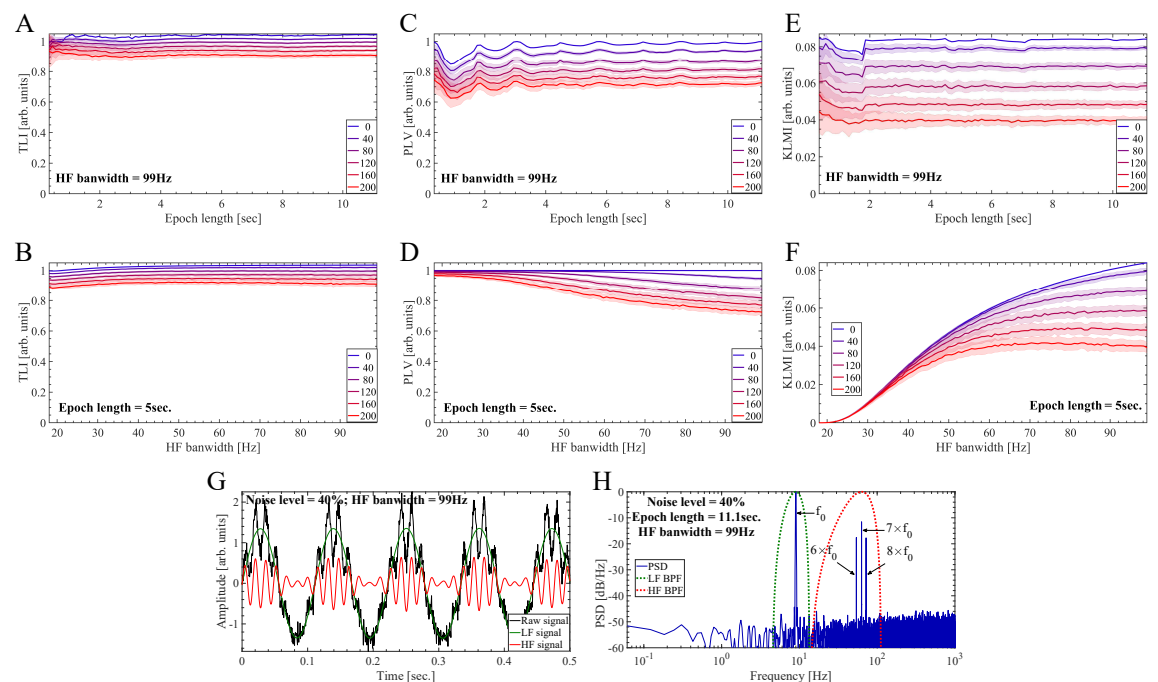

Figure 8: Performance of harmonicity (TLI, $\left.P L V_{P P C}\right)$ and PAC $\left(K L M I_{P A C}\right)$ metrics to characterize an amplitude modulated synthetic signal. In all the cases shown in this figure, we used a sampling rate of $f_{s}=2000 \mathrm{~Hz}$ and the frequency and amplitude of the modulating $(\mathrm{LF})$ and the amplitude-modulated (HF) oscillations were kept unchanged. To obtain all the band-pass filtered signals shown in this figure we use the BPF as described in Appendix A.5 The bandwidth of the BPF for the LF component (LF BPF) was kept fixed at $B w_{L F}=9$ Hz. The $P L V_{P P C}$ was computed using Eq. 4 with the configuration given by Eq. A.19 and $M=1, N=7$. The amplitude-modulated signal was synthesized as described in Appendix A.1 using the following hyperpameter values: $c=1$ (i.e. DSB-C), maximum modulation depth $m=0, \eta_{m}=0$, we used a sinusoidal modulating $a(t)$ at $f_{0}=f_{L F}=9 \mathrm{~Hz}$ as given by Eq. A.6 $A_{m}=1, \phi_{m}=0, z_{D B S}$ was set with $f_{H F}=7 \times f_{L F}=63 \mathrm{~Hz}, \phi_{c}=0$, $z_{H F}=0$, for $z_{h}$ we use $A_{1}=4, A_{k}=0 \forall k>1$ and $\phi_{k}=0 \forall k$. In Eq. A.1. we configured a constant amplitude envelope $\mathcal{E}(t)=1$. The extrinsic noise level shown in the graphs corresponds to $\eta(t)$ in Eq. A.1 and is expressed as the percent of the modulating signal $a(t)$ maximum amplitude $\left(A_{m}\right)$ scaling the standard deviation $\sigma$ of the additive white Gaussian noise $(\mathrm{AWGN}) \eta \approx \mathcal{N}(0, \sigma)$. (A, C, E) Harmonicity (TLI, PLV $V_{P C C}$ ) and PAC $\left(K L M I_{P A C}\right)$ metrics as a function of the epoch length and taking the level of AWGN as a parameter. To compute graphs A, C and E, the bandwidth of the HF BPF was kept unchanged in $B w_{H F}=99 \mathrm{~Hz}$. Our implementation of the TLI algorithm (Section 2.4 requires at least 3 cycles of the low frequency oscillation $\left(f_{L F}=9 \mathrm{~Hz}\right)$, which determines the minimum epoch length shown in graphs $\mathrm{A}$ and $\mathrm{D}\left(3 / f_{L F} \approx 0.3 \mathrm{sec}\right.$.). The maximum epoch length used to compute graphs $\mathrm{A}$ and $\mathrm{D}$ was $100 / f_{L F} \approx 11.1$ sec. (B, D, F) Harmonicity (TLI, PLV $V_{P P C}$ ) and PAC $\left(K L M I_{P A C}\right)$ metrics as a function of the HF bandwidth $\left(B w_{H F}\right)$ corresponding to the BPF used to obtain the HF signal $\left(x_{H F}(t)\right)$, and taking the level AWGN as a parameter. The minimum and maximum $B w_{H F}$ values used to compute the graphs $\mathrm{B}$ and $\mathrm{E}$ were 18 $\mathrm{Hz}$ and $99 \mathrm{~Hz}$, respectively. To compute the graphs $\mathrm{B}, \mathrm{D}$ and $\mathrm{F}$, the epoch length was kept unchanged in $45 / f_{L F} \approx 5$ sec. In the panels $\mathrm{A}, \mathrm{B}, \mathrm{C}, \mathrm{D}, \mathrm{E}$ and $\mathrm{F}$, the solid lines represent the mean values and the shaded error bars correspond to the standard deviation of 100 realizations at each point. (G) Synthetic dynamics (solid black line) together with the HF and LF signals shown as solid red and green lines, respectively. The synthetic dynamics includes additive white Gaussian noise $\mathcal{N}(0, \sigma)$ with the standard deviation $\sigma$ corresponding to the $40 \%$ of the modulating signal $a(t)$ maximum amplitude $\left(A_{m}\right)$. The LF and HF signals where obtained by filtering the raw signal with the band-pass filters whose power responses are shown as dotted green $\left(B w_{L F}=9 \mathrm{~Hz}\right)$ and red $\left(B w_{H F}=99 \mathrm{~Hz}\right)$ lines in graph $\mathrm{H}$, respectively. (H) Power spectrum (solid blue line) of the synthetic dynamics (solid black line in graph G) computed using an epoch length of $100 / f_{L F} \approx 11.1 \mathrm{sec}$. The power responses (i.e. square magnitude) of the BPF used to compute the LF and HF signals are shown as dotted green and red lines, respectively. 
As a conclusion, it was found that for dynamics with two harmonic (Figures 5 to 8 or two non harmonic (Figure B.1) oscillatory components, the TLI and $P L V_{P P C}$ metrics have a comparable performance in terms of the explored parameters. On the other hand, for oscillatory dynamics containing multi-harmonic HF components the TLI present a better performance when compared with the $P L V_{P P C}$ metric under similar conditions (compare panels $\mathrm{A}$ and $\mathrm{C}$ of Figure 8). This aspect will be further discussed below in connection with the simulated dynamics of the Van der Pol oscillator.

The TLI metric was tailored designed to be combined with the PC metric for improving the characterization and interpretation of the CFC patterns observed at the signal level. To illustrate this point, the temporal evolution of the relevant metrics were analyzed during a variety of synthetic oscillatory dynamics. For this, time series for the PLV, KLMI, TLI and PC metrics were constructed as it was described in Section 2.8. It is essential to note a key point regarding the TLI temporal evolution as a complementary tool to interpret the estimators aimed to quantify CFC (e.g. PLV, MVL, KLMI). Even though the TLI is a measure bounded in the range $[0,1]$ (see Section 2.4) and independent of the processed oscillations amplitude, the absolute value of the TLI does depend on the noise level present in the processed time series and on the epoch length, i.e. the number of periods of the low frequency oscillation taken to implement the time-locked average involved in the TLI computation (see Figures 5 to 8 and B.1). A similar behavior was observed for the bounded (PLV, KLMI $\in[0,1]$ ) and unbounded (MVL) CFC metrics. As a consequence, a robust indicator of the occurrence of transient harmonic CFC patterns is given by the fact that the TLI increases concurrently with the CFC metrics, rather than by the absolute TLI value at a particular time instant. In this regard, Figure $9 \mathrm{~A}$ shows a synthetic dynamics presenting a transient harmonic PAC pattern. This type of transient dynamics is relevant since it is commonly observed during the ictal activity recorded invasively in patients candidates to epilepsy surgery and animal models of epilepsy (see [1] and references therein). The dynamics was synthesized using Eqs. A.1. A.4 configured for the DSB-C case with a Gaussian modulating $a(t)$ (Eqs. A.7 and A.8) and maximum modulation depth (see the caption of Figure 9 for the complete list of parameter values). The transient harmonic PAC pattern was implemented through the time series envelope $\mathcal{E}(t)$ as defined in Eqs. A.2 and A.3. Figure 9B shows that the PAC $\left(P L V_{P A C}\right)$ and harmonicity (TLI) metrics increase almost concurrently from their baseline value previous to the transient activation to close the unity. Note that while the amplitude-modulated dynamics remains stable $(80$ sec. $\lesssim$ Time $\lesssim 120$ sec. in Figure 9 $\mathrm{A}$ ) so the PAC and harmonicity metrics indicating the occurrence of a PAC pattern $\left(P L V_{P A C} \approx 1\right)$ constituted by harmonic spectral components $(T L I \approx 1)$ which is not an ephiphenomenon due to the presence of phase clustering $\left(P C_{L F} \approx 0\right)$. Figures $9 \mathrm{D}$ and $9 \mathrm{E}$ show the signals and power spectrum representative of this time interval in which the dynamics remains stable. In particular, 9D shows the modulating signal with $f_{L F}=3 \mathrm{~Hz}$ (solid green line, $\left.f_{L F}=3 \mathrm{~Hz}\right)$ and modulated $\left(f_{H F}=89 \times f_{L F}=267 \mathrm{~Hz}\right.$, red solid line) signals obtained band-pass filtering the raw dynamics (solid black line). The modulat- 
${ }_{630}$ ing and modulated signals were computed using the LF BPF (dotted green line) ${ }_{631}$ and HF BPF (dotted red line) shown in Figure 9E, respectively. In addition, the ${ }_{632}$ harmonicity map and comodulogram computed for an epoch during the time ${ }_{633}$ interval in which the dynamics remains stable are shown in Figures $9 \mathrm{C}$ and $9 \mathrm{~F}$, ${ }_{634}$ respectively, revealing the modulating $\left(f_{L F}\right)$ and modulated $\left(f_{H F}\right)$ frequency ${ }_{635}$ bands involved in the harmonic PAC pattern. 
bioRxiv preprint doi: https://doi.org/10.1101/2020.10.15.341800; this version posted November 1, 2020. The copyright holder for this preprint (which was not certified by peer review) is the author/funder, who has granted bioRxiv a license to display the preprint in perpetuity. It is made available under aCC-BY-NC-ND 4.0 International license.

A
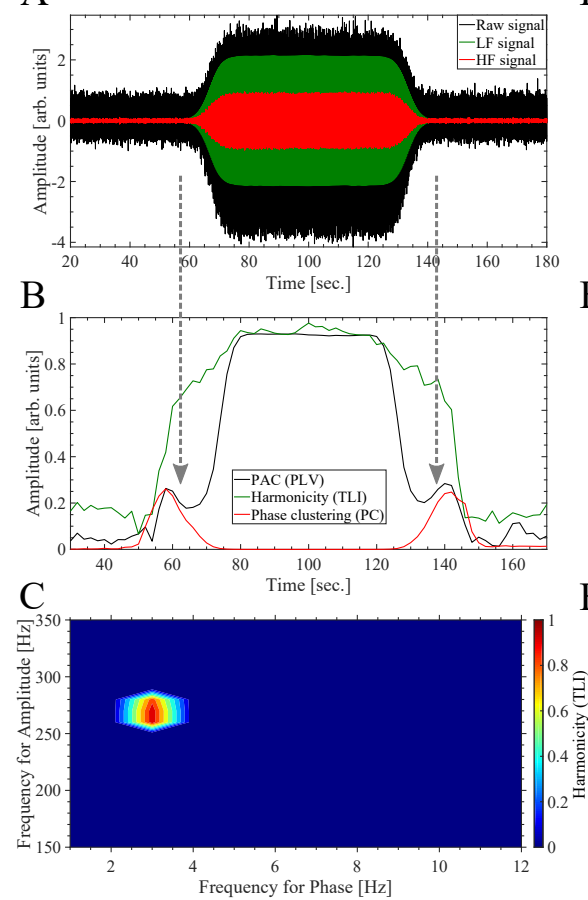

$\mathrm{D}$

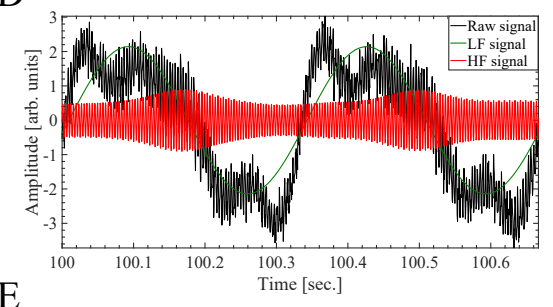

$\mathrm{E}$
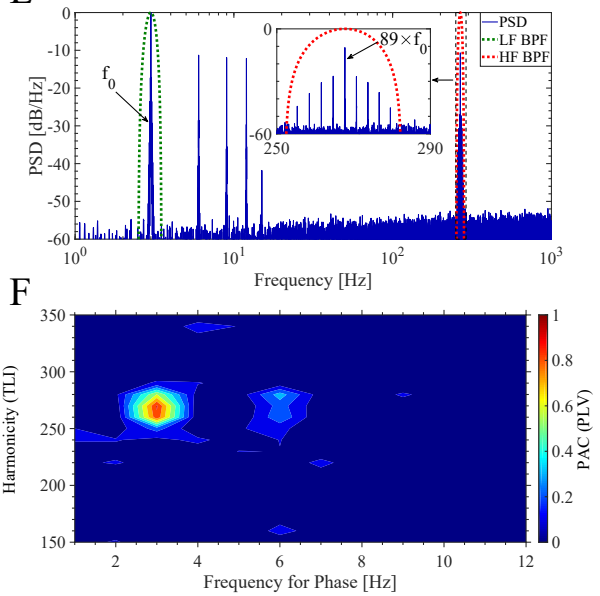

Figure 9: Temporal evolution of the PAC $\left(P L V_{P A C}\right)$, harmonicity (TLI) and phase clustering $\left(P C_{L F}\right)$ metrics during a synthetic dynamics presenting a transient harmonic PAC pattern. To obtain all the band-pass filtered signals shown in this figure we use the BPF as described in Appendix A.5 (A) Synthetic dynamics (solid black line) together with the HF and LF signals shown as solid red and green lines, respectively. The dynamics (solid black line) was synthesized using Eqs. A.1 and A.4 with the following hyperpameter values: sampling rate $f_{s}=2000 \mathrm{~Hz}, c=1$ (i.e. DSB-C), half modulation depth $m=0.5, \eta_{m}=0$, we used a Gaussian modulating $a(t)$ with the fundamental frequency at $f_{0}=f_{L F}=3 \mathrm{~Hz}$ as given by Eqs. A.7 and A.8 with $\sigma \approx 55$ and $A_{m}=1, z_{D B S}$ was set with $f_{H F}=89 \times f_{L F}=267$ $\mathrm{Hz}, \phi_{c}=0, z_{H F}=0$, for $z_{h}$ we use $A_{1}=4, A_{k}=1 \forall 2 \leq k \leq 4, A_{k}=0 \forall k \geq 5$ and $\phi_{k}=0 \forall k$. The transient harmonic PAC pattern was implemented through the time series envelope $\mathcal{E}(t)$ as defined in Eqs. A.2 and A.3 with $\alpha=0.5$ and $\beta$ equals to one third of the time series length. Extrinsic noise $\eta(t)$ was added as shown in Eq. A.1 In this case the noise level corresponds to the 10 percent of the maximum amplitude of the deterministic part of signal $x(t)$ (i.e first term of the right-hand member of the Eq. A.1, scaling the standard deviation $\sigma$ of the additive white Gaussian noise (AWGN) $\eta \approx \mathcal{N}(0, \sigma)$. The LF (solid green line) and HF (solid red line) signals where obtained by filtering the raw signal (solid black line) with the band-pass filters whose power responses are shown as dotted green $\left(B w_{L F}=1 \mathrm{~Hz}\right)$ and red $\left(B w_{H F}=30 \mathrm{~Hz}\right)$ lines in graph $\mathrm{E}$, respectively. (B) Time series showing the temporal evolution of the $P L V_{P A C}$, TLI and $P C_{L F}$ metrics. These time series were computed as described in Section 2.8 using the algorithm 2 summarized in Table 2 with a sliding window of $20 \mathrm{sec}$. in length, i.e. 60 cycles of the slowest oscillatory component at $f_{0}=f_{L F}=3 \mathrm{~Hz}$. (C) TLI harmonicity map computed as described in Section 2.7 using a 20 sec. epoch extracted from the center (Time $\approx 100 \mathrm{sec}$.) of the synthetic dynamics shown in panel A. In computing the map, all the TLI values below the significance threshold were set to zero (see Section 2.7. The pseudocolor scale represents the TLI values ranging from 0 (blue) to 1 (red). (D) Zoom showing two cycles of the synthetic dynamics (solid black line) together with the HF and LF signals shown as solid red and green lines, respectively. The two cycle epoch corresponds to the center (Time $\approx 100 \mathrm{sec}$.) of the synthetic dynamics shown in panel A. (E) Power spectrum (solid blue line) computed from the synthetic dynamics (solid black line in graph A). The power responses (i.e. square magnitude) of the BPF used to compute the LF and HF signals are shown asgdotted green and red lines, respectively. (F) Comodulogram computed as described in Section 2.7 computed from the same epoch used to obtain the harmonicity map (panel C). In computing the comodulogram, all the $\left|P L V_{P A C}\right|$ values below the significance threshold were set to zero (see Section 2.7). The pseudocolor scale represents the $\left|P L V_{P A C}\right|$ values ranging from 0 (blue) to 1 (red). The harmonicity map (panel C) and comodulogram (panel F) were computed using the same BPF (see Appendix A.5. 
Due to the fact that to compute the metrics shown in Figure 9 we used sufficiently narrow LF BPF to obtain an almost sinusoidal low frequency component (dotted green line in Figure $9 \mathrm{E}$ and solid green line in Figure 9D), i.e. uniform distribution of $\phi_{L F}(t)$ values in Eq. 8 the $\left|P C_{L F}\right|$ time series is close to zero along the transient dynamics (red solid line in Figure 9B). This indicates that the observed PAC pattern is not a spurious artifacts related to the presence of phase clustering in the modulating LF component [17, 18]. On the other hand, Figure 10 corresponds to the very same synthetic dynamics of that shown in Figure 9A, but in this case we use a wide LF BPF including several spectral components, and thus, resulting in a highly non sinusoidal low frequency component (dotted green line in Figure $10 \mathrm{E}$ and solid green line in Figure 10D). As a consequence, we obtain a skewed distribution of phase angles producing $\left|P C_{L F}\right| \approx 0.5$. It is crucial to note that this finite phase clustering associated to a non sinusoidal low frequency component $\left(P C_{L F}\right)$ produces a bias in both the $\mathrm{PAC}$ and harmonicity metrics, which in this case becomes evident by comparing Figures $9 \mathrm{~B}$ and $10 \mathrm{~B}$. Note that a wider LF BPF (dotted green line in Figure $10 \mathrm{E}$ ) imposes a limit on the minimum value of the frequency for phase (abscissa) that is possible to compute in the harmonicity map and comodulogram as it is shown in Figures $10 \mathrm{C}$ and $10 \mathrm{~F}$, respectively. 
A

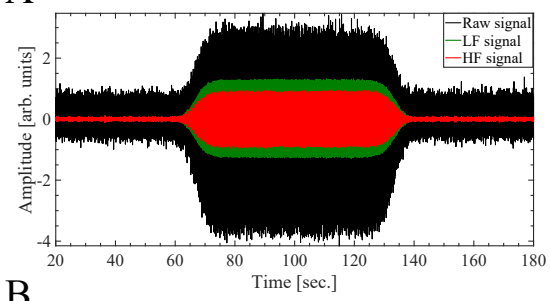

B
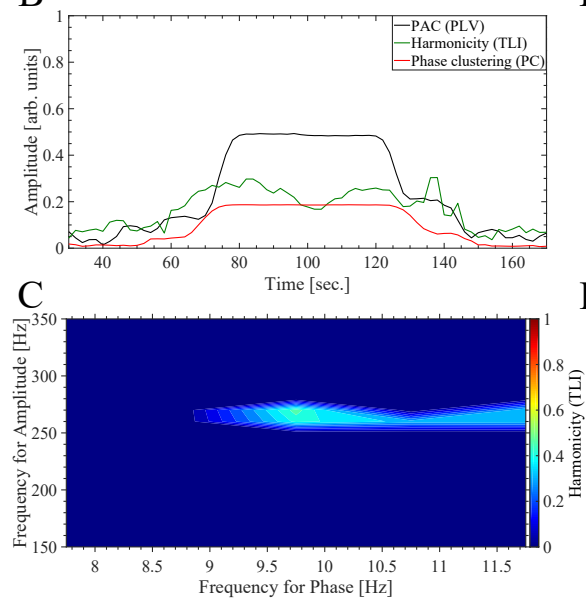

$\mathrm{D}$

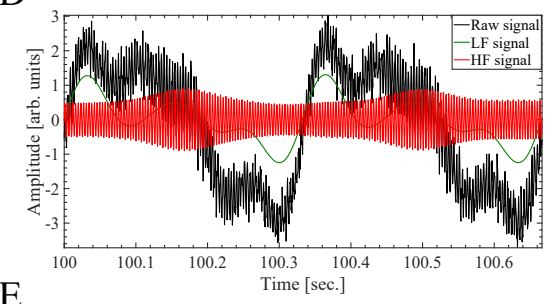

$\mathrm{E}$

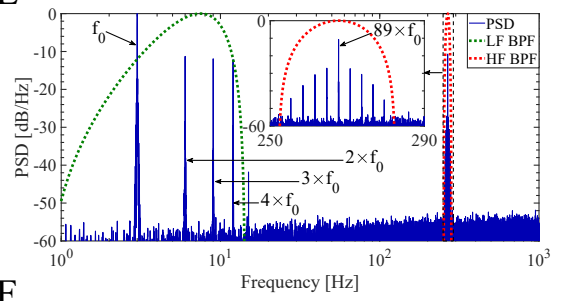

$\mathrm{F}$

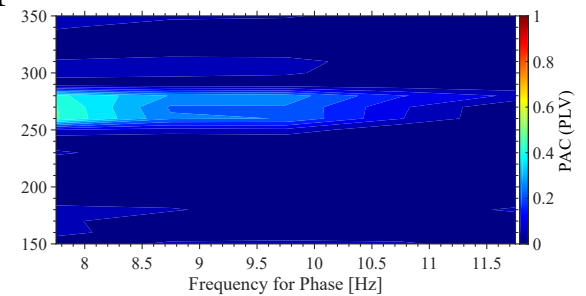

Figure 10: Temporal evolution of the PAC $\left(P L V_{P A C}\right)$, harmonicity (TLI) and phase clustering $\left(P C_{L F}\right)$ metrics during a synthetic dynamics presenting a transient harmonic PAC pattern. In this plot we use the same synthetic dynamics and the same set of hyperparameter values to compute the metrics than those described in the caption of Figure 9 except for the bandwidth of the BPF used to compute the LF component $\left(B w_{L F}\right)$. In this case, the $P L V_{P A C}$, TLI and $P C_{L F}$ metrics were computed using $B w_{L F}=13.5 \mathrm{~Hz}$ centered around $7.5 \mathrm{~Hz}$ (see the dotted green line in panel E). This wide BPF produces a non sinusoidal LF component (see solid green line in panel D), characterized by a non uniform distribution of phase values producing the increase of the phase clustering $\left(P C_{L F}\right)$ during the dynamics (see solid red line in panel B). Note the bias in the PAC ( $\left.P L V_{P A C}\right)$ and harmonicity (TLI) metrics due to the presence of phase clustering $\left(P C_{L F}\right)$. The description of the panels is the same than that given in Figure 9

In Appendix B.2 we present the behavior of the harmonicity and PAC metrics during a variety of transient dynamics (e.g. non harmonic PAC), and also discuss the bias produced on the MVL metric $\left(M V L_{P A C}\right)$ by the phase clustering associated to a non sinusoidal low frequency component $\left(P C_{L F}\right)$.

It was found that the abrupt change of the raw signal amplitude associated to transient dynamics like those shown in Figures 9, 10, B.2 to B.5, is capable to produce spurious CFC values due to the interaction between the sliding epoch and the abrupt change of the amplitude envelope of the raw time series (see grey arrows in panels A and B of Figure 9). Importantly, these spurious CFC values at rising and falling edges of the transient dynamics are effectively detected by the $P C_{L F}$ metrics since they occurs concomitantly with an increase of the phase clustering. On the other hand, due to the fact that the TLI is an amplitude 
independent quantity sensitive only to PPC, it does not present these artifacts associated to changes in the amplitude of the analyzed dynamics (see the TLI time series in Figures 9 , 10, B.2 to B.5.

We identify another confounding associated to the Algorithm 1 described in Table 2 for the computation of CFC time series. Specifically, Figures 11A and 111C show the $P L V_{P A C}$ and $P C_{L F}$ time series computed with the algorithms described in Table 2 together with the TLI metric for a transient harmonic PAC pattern similar to that shown in Figure 9. We found that using short sliding epochs of about 10 cycles of the slowest oscillation, Algorithm 1 produce time series of PAC metrics (e.g. $P L V_{P A C}$ ) which are monotonically decreasing toward and away from the rising and falling edges of the transient dynamics (see grey arrows in Figure 11A). Besides, Figure 11B shows that this effect is also distinguishable in the case of a transient oscillatory dynamics without PAC similar to that shown in Figure B.2. We observed this behavior of the CFC time series computed via the Algorithm 1 in a variety of transient oscillatory dynamics, suggesting that is a confounding strongly related to the abrupt change of the amplitude envelope of the raw time series, irrespective of the type and intensity of the CFC present in the dynamics. We emphasize that this confounding is particularly dangerous since it seems not to be associated to an concomitantly increase of the phase clustering time series, and therefore it is difficult to detected (see red solid line in Figures $11 \mathrm{~A}$ and $11 \mathrm{~B}$ ). On the other hand, Figures 11C and 11D show that the monotonically decreasing trend is absent in the time series of PAC metrics computed using the Algorithm 2. Moreover, when comparing Figures $111 \mathrm{~A}$ and $11 \mathrm{C}$ it becomes evident the bias introduced by the Algorithm 1 in the maximum intensity of the $P L V_{P A C}$ time series. We identify the root cause of these confounding as the computation of features (e.g. phase, amplitude, frequency) via the Hilbert transform on the whole band-pass filtered time series including the abrupt changes of amplitude associated to the rising and falling edges of the transient dynamics, which affect the resulting features (see step 3 in Algorithm 1 of Table 2). On the other hand, these issues are avoided in the Algorithm 2 of Table 2 by first dividing the band-pass filtered signals in sliding epochs, the resulting epochs are Z-scored to make them independent of the absolute amplitude of the filtered signals and then the features are computed by applying the Hilbert transform on the Z-scored epochs (see step 4 and 5 in Algorithm 2 of Table 2).

The behavior of time series of PAC metrics shown in Figures 11A and 11B associated to the confounding of Algorithm 1 has been also observed during the transition between the pre-ictal to ictal periods in intracerebral electroencephalography recordings (LFP: local field potential) obtained from the seizure onset zone of epilepsy patients [11] (data not shown). This result is relevant since several CFC types, in particular PAC, have been proposed as biomarkers for detecting the seizure onset in epilepsy patients. As a conclusion, our results suggest that Algorithm 1 should be avoided in analyzing oscillatory dynamics characterized by abrupt changes of amplitude, where the Algorithm 2 is recommended instead. In addition, the temporal evolution of CFC metrics around transient dynamics involving abrupt changes of amplitude (or any other feature), 

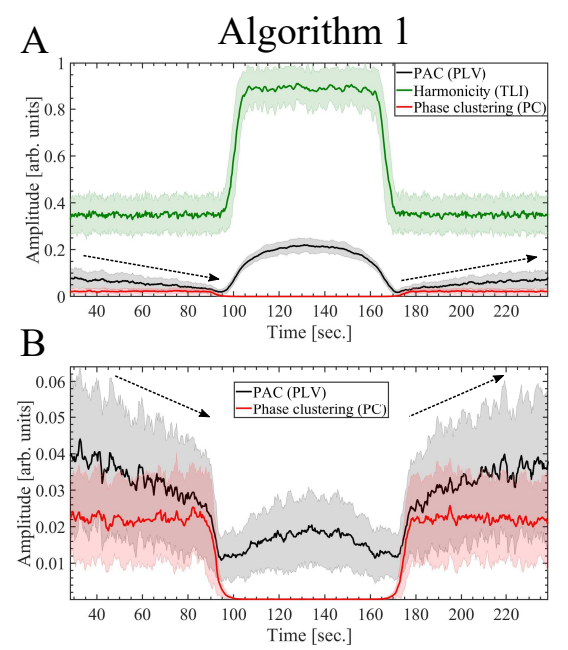

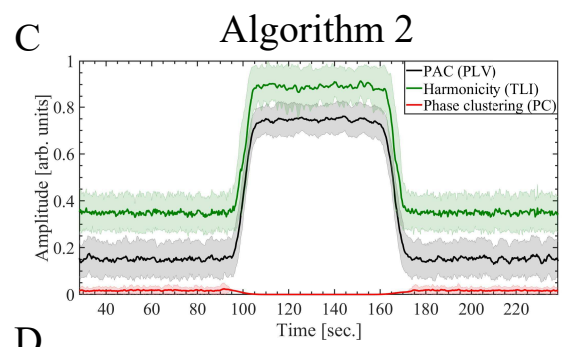

$\mathrm{D}$

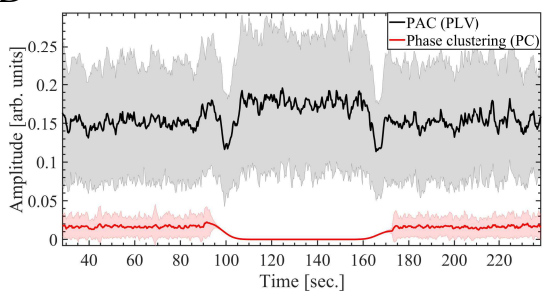

Figure 11: Comparison of the algorithms 1 and 2 are summarized in Table 2 aimed to compute time series of CFC metrics. The time series showing the temporal evolution of the PLVPAC, TLI and $P C_{L F}$ metrics were computed as described in Section 2.8 using a sliding window of $3.33 \mathrm{sec}$. in length, i.e. 10 cycles of the slowest oscillatory component at $f_{0}=f_{L F}=3 \mathrm{~Hz}$. $(\mathrm{A}, \mathrm{C})$ The time series shown in panels $\mathrm{A}$ and $\mathrm{C}$ were computed from a synthetic dynamics presenting a transient harmonic PAC pattern, as described in Section 2.8 using the algorithms 1 and 2, respectively. The synthetic dynamics used in panels $\mathrm{A}$ and $\mathrm{C}$ was computed using the same set of hyperparameter values as those described in the caption of Figure 9 with the exception of the extrinsic noise $\eta(t)$ which in this case was set to 30 percent of the maximum amplitude of the deterministic part of signal $x(t)$ (i.e first term of the right-hand member of the Eq. A.1. (B, D) The time series shown in panels B and D were computed from a synthetic dynamics without PAC, as described in Section 2.8 using the algorithms 1 and 2, respectively. The synthetic dynamics used in panels $B$ and $D$ was computed using the same set of hyperparameter values as those described in the caption of Figure B.2 with the exception of the extrinsic noise $\eta(t)$ which in this case was set to 30 percent of the maximum amplitude of the deterministic part of signal $x(t)$ (i.e first term of the right-hand member of the Eq. A.1. In the panels A, B, C and D, the solid lines represent the mean values and the shaded error bars correspond to the standard deviation of 100 realizations at each point.

We characterized quantitatively the harmonic content of CFC patterns using the harmonicity-CFC plots which categorize the analyzed oscillatory dynamics in four quadrants, Q1: harmonic CFC, Q2: harmonic oscillations and No CFC, Q3: Non harmonic oscillations and No CFC, Q4: Non harmonic CFC. Figure 12 shows the harmonicity-PAC plot, computed as it was described in Section 
2.6. for a variety of synthetic oscillatory dynamics and taking the amplitude modulation depth as a parameter.
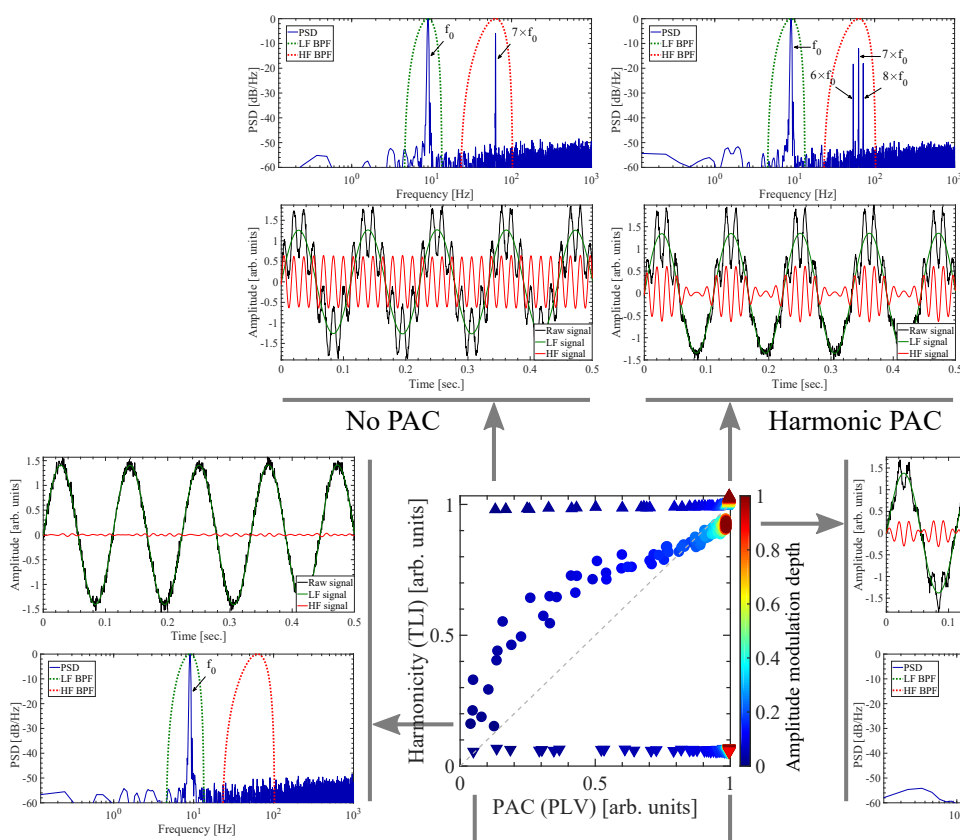

Harmonic PAC
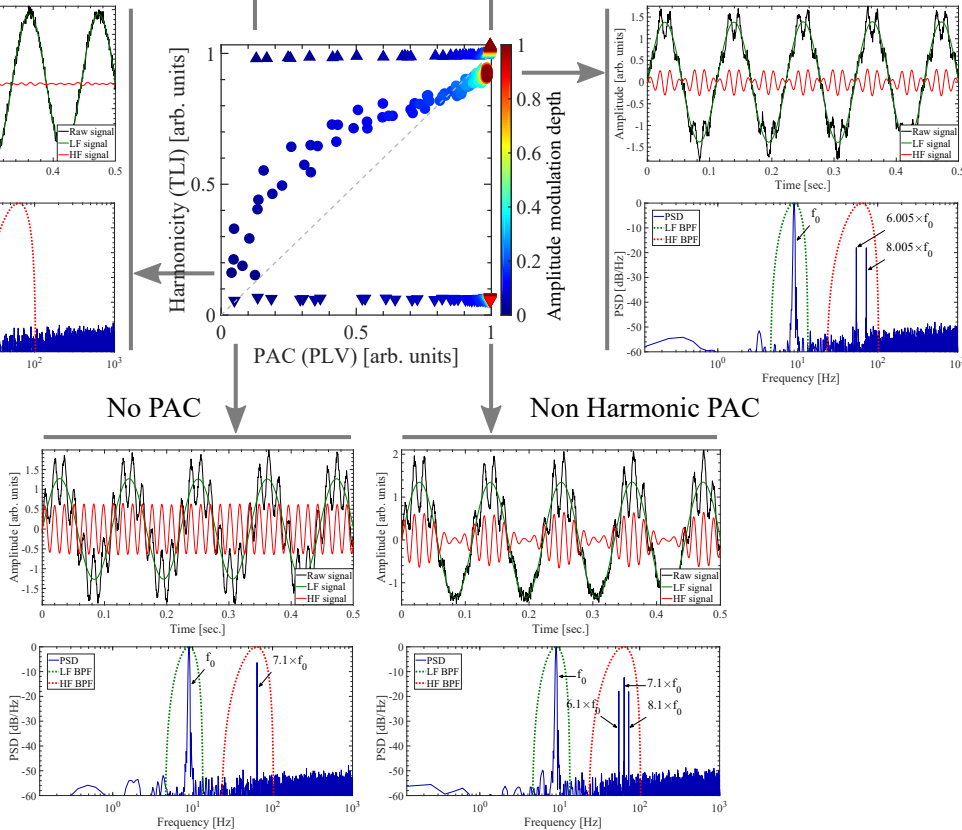

Figure 12: Harmonicity-PAC plot computed for a variety of synthetic oscillatory dynamics and taking the amplitude modulation depth as a parameter. The Harmonicity-PAC plot was computed as it is described in Section 2.6 The pseudocolor scale represents the $1-m$ values ranging from the minimum 0 (blue) to the maximum 1 (red) modulation depth, with $m$ defining the amplitude modulation depth as stated in Eq. A.4 The synthetic dynamics were computed as it is described in Appendix A.1 using sinusoidal modulating signals (Eq. A.6.

3.2. A single non sinusoidal oscillatory dynamics characterized by dependent frequencies

In this section we investigate the robustness of the proposed (TLI) and conventional $\left(P L V_{P P C}\right)$ harmonicity measures to quantify the harmonic content of the simulated dynamics of the Van der Pol oscillator (see Appendix A.2). 
We shall show that, contrary to what is usually assumed in the literature, the spurious PAC elicited by a single non sinusoidal oscillatory dynamics like the one associated to the Van der Pol oscillator can produce both harmonic and non harmonic PAC patterns.

Figure 13 shows that, in the case of oscillatory dynamics containing multiharmonic HF components, the TLI is more robust than the $P L V_{P P C}$ against changes in the bandwidth of the BPF used to compute the high frequency component (HF BPF, see the dotted and solid red lines in panels A, B, C and $\mathrm{D}$ of Figure 13). The non sinusoidal oscillatory dynamics shown in Figure 13 . is constituted by a fundamental component at $f_{d}=5.56 \mathrm{~Hz}$ and odd harmonic components at $N \times f_{0}$ with $N=3,5,7,9,11,13, \cdots$. In Figure 13 the bandpass filters used to compute the harmonicity metrics were centered at $f_{L F}=$ $1 \times 5.56 \mathrm{~Hz}(\mathrm{LF} \mathrm{BPF})$ and $f_{H F}=9 \times 5.56 \mathrm{~Hz}(\mathrm{HF} \mathrm{BPF})$, and consequently, the $P L V_{P P C}$ metric was computed using Eq. 4 with $M=1, N=9$. Figure $13 \mathrm{E}$ shows that the drop of the $P L V_{P P C}$ value occurs concurrently with the increase of the phase clustering $P C_{H F}$, indicating that the former is produced by a non uniform distribution of the phase values associated to the HF component, i.e. non sinusoidal $x_{H F}$ (solid red line in Figure $13 \mathrm{D}$ ). Worthy to note, Figure $13 \mathrm{E}$ also shows that increments of the $\mathrm{HF}$ bandwidth up to $B w_{H F} \approx 20 \mathrm{~Hz}$ degrade the signal-to-noise ratio in the band-pass filtered signal $x_{H F}$ producing a moderate drop of the TLI value. On the other hand, for $B w_{H F} \gtrsim 20 \mathrm{~Hz}$, the HF bandwidth is wide enough to include other harmonic components and thus improving the signal-to-noise ratio of $x_{H F}$ which translates in that the TLI values become closer to the unity again. Importantly, we found that the pairwise phase consistency measure [23, 24] is also affected by the phase clustering $P C_{H F}$ presenting a similar behavior to that shown by the $P L V_{P P C}$ metric in Figure $13 \mathrm{E}$ (data not shown). This results is not surprising since the pairwise phase consistency measure was algorithmically derived from the PLV metric 23]. 

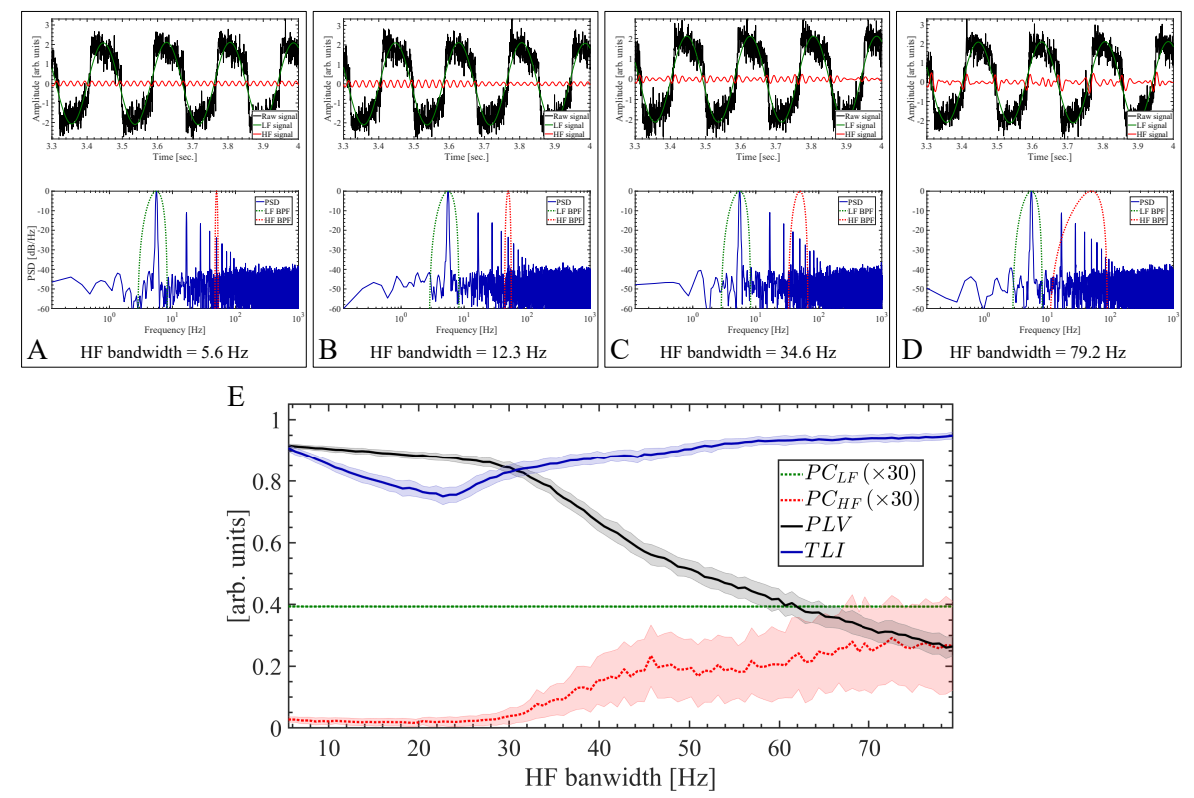

Figure 13: The TLI is more robust than the $P L V_{P P C}$ against changes in the bandwidth of the $\mathrm{BPF}$ used to compute the high frequency component. The Van der Pol oscillatory dynamics (solid black line) shown in panels A, B, C and D were simulated as described in Appendix A.2 using the following hyperpameter values: sampling rate $f_{s}=2000 \mathrm{~Hz}, \mu=300, \omega_{0}=2 \pi f_{0}$, $f_{0}=10 \mathrm{~Hz}, W_{p}=0, F_{e}=0$, initial conditions $x(0)=2, \dot{x}(0)=1$. With this configuration, we obtain a non sinusoidal oscillatory dynamics constituted by a fundamental component at $f_{d}=5.56 \mathrm{~Hz}$ and odd harmonic components at $N \times f_{d}$ with $N=3,5,7,9,11,13, \cdots$. The dynamics was computed without intrinsic noise $\left(g_{1}=g_{2}=0\right.$ in Eq. A.14 in order to obtain a non sinusoidal oscillatory dynamics with a constant fundamental period. Extrinsic noise $\eta(t)$ was added as shown in Eq. A.14 In this case the noise level corresponds to the 20 percent of the maximum amplitude of the deterministic part of signal (i.e $x_{1}(t)$ in Eq. A.14), scaling the standard deviation $\sigma$ of the additive white Gaussian noise (AWGN) $\eta \approx \mathcal{N}(0, \sigma)$. We computed the Van der Pol dynamics for 6 sec. time interval and then the first $0.1 \mathrm{sec}$. (200 samples) of the time series were discarded to remove the transient period of the numerical simulation. For this set of hyperparameter values, panels A, B, C, D show different realizations of the Van der Pol dynamics. To obtain all the band-pass filtered signals shown in this figure we use the BPF as described in Appendix A.5 The bandwidth of the BPF for the LF component (LF BPF) was kept fixed at $B w_{L F}=f_{d}=5.56 \mathrm{~Hz}$ (see the dotted green lines superimposed to the power spectra shown in panels A, B, C, D). The band-pass filters used to compute the harmonicity metrics were centered at $f_{L F}=1 \times f_{d} \mathrm{~Hz}(\mathrm{LF} \mathrm{BPF})$ and $f_{H F}=9 \times f_{d} \mathrm{~Hz}(\mathrm{HF}$ $\mathrm{BPF})$, and consequently, the $P L V_{P P C}$ metric was computed using Eq. 4 with $M=1, N=9$. In panels $\mathrm{A}, \mathrm{B}, \mathrm{C}, \mathrm{D}$ we changed the bandwidth of the $\mathrm{BPF}$ for the HF component (HF BPF) whose power response (i.e. square magnitude) is shown as dotted red line superimposed to the power spectra. The resulting band-pass filtered HF signals are shown as solid red lines in the upper graph of panels A, B, C, D. The power spectra and the harmonicity metrics (TLI, $\left.P L V_{P P C}\right)$ were computed using an epoch length of $\approx 33 / f_{d} \approx 6$ sec. The panel (E) shows the magnitude of the harmonicity (TLI, $\left.P L V_{P P C}\right)$ and phase clustering metrics $\left(P C_{L F}, P C_{H F}\right)$ as a function of the bandwidth of the BPF for the HF component (HF BPF). In the panel E, the solid lines represent the mean values and the shaded error bars correspond to the standard deviation of 100 realizations at each HF bandwidth value.

We also investigate the effect of the bandwidth associated to the BPF used to 
compute the low frequency component (LF BPF, see the dotted and solid green lines in panels $\mathrm{A}, \mathrm{B}$ and $\mathrm{C}$ of Figure 14). In this case the filters were centered at $f_{L F}=5 \times 5.56 \mathrm{~Hz}(\mathrm{LF} \mathrm{BPF})$ and $f_{H F}=15 \times 5.56 \mathrm{~Hz}(\mathrm{HF} \mathrm{BPF})$, and the $P L V_{P P C}$ metric was computed using Eq. 4 with $M=5, N=15$. Figure 14D shows that both harmonicity metrics are degraded by the increase of the phase clustering $P C_{L F}$ associated to a non sinusoidal low frequency component $x_{L F}$ (see the solid green line in Figure $14 \mathrm{C}$ ). That is, as the $B w_{L F}$ is increased to include several harmonic components within its bandwidth, a non sinusoidal LF components is obtained at the output of the LF BPF filter (see the solid green line in Figure $14 \mathrm{C}$ ). This in turns produces an increment in the phase clustering $\left(P C_{L F}\right)$, biasing the intensity of both metrics (TLI, $\left.P L V_{P P C}\right)$ toward values close to zero. Un this condition, both harmonicity metrics (TLI, $P L V_{P P C}$ ) fail to detect the presence of harmonic components in the oscillatory dynamics. 


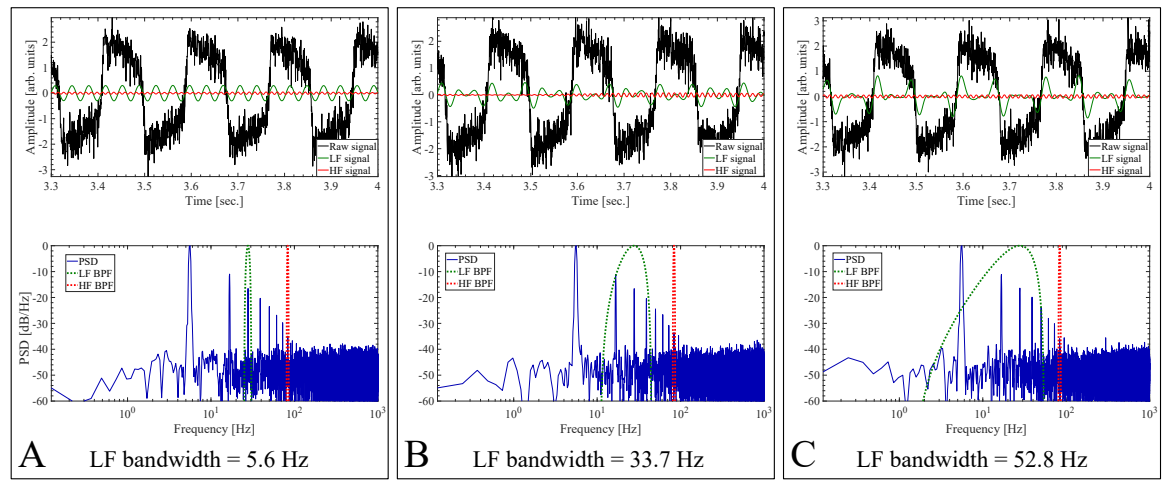

$\mathrm{D}$

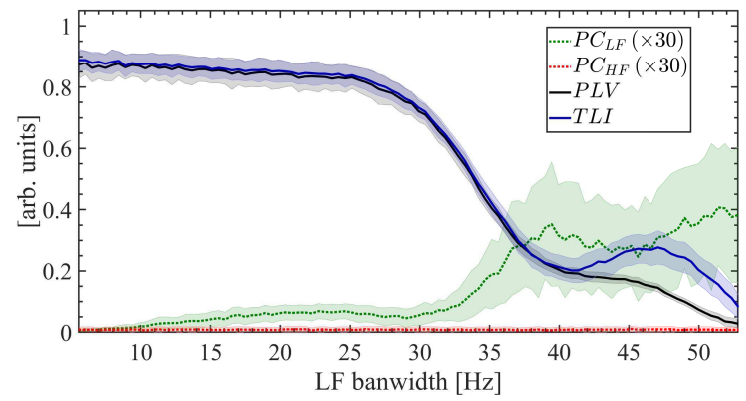

Figure 14: The phase clustering associated to the low frequency component $\left(P C_{L F}\right)$ produces a bias in both TLI and $P L V_{P P C}$ metrics. In this figure we use the same synthetic dynamics and the same set of hyperparameter values to compute the metrics than those described in the caption of Figure 13 except for the configuration of the BPFs used to compute the LF and HF components. In this case, the bandwidth of the BPF for the HF component (HF BPF) was kept fixed at $B w_{H F}=f_{d}=5.56 \mathrm{~Hz}$ (see the dotted red lines superimposed to the power spectra shown in panels A, B, C). Besides, the band-pass filters used to compute the harmonicity metrics were centered at $f_{L F}=5 \times f_{d} \mathrm{~Hz}(\mathrm{LF} B P F)$ and $f_{H F}=15 \times f_{d} \mathrm{~Hz}(\mathrm{HF}$ $\mathrm{BPF}$ ), and consequently, the $P L V_{P P C}$ metric was computed using Eq. 4 with $M=5, N=15$. In panels $\mathrm{A}, \mathrm{B}, \mathrm{C}$ we changed the bandwidth of the BPF for the LF component (LF BPF) whose power response (i.e. square magnitude) is shown as dotted green line superimposed to the power spectra. The resulting band-pass filtered LF signals are shown as solid green lines in the upper graph of panels A, B, C. The power spectra and the harmonicity metrics (TLI, $\left.P L V_{P P C}\right)$ were computed using an epoch length of $\approx 33 / f_{d} \approx 6$ sec. The panel D shows the magnitude of the harmonicity (TLI, $\left.P L V_{P P C}\right)$ and phase clustering metrics $\left(P C_{L F}, P C_{H F}\right)$ as a function of the bandwidth of the BPF for the LF component (LF BPF). In the panel D, the solid lines represent the mean values and the shaded error bars correspond to the standard deviation of 100 realizations at each LF bandwidth value.

Any non linear oscillator can be used as a model that generates spurious PAC via separation of time scales due to non linear effects. Importantly, these emerging time scales elicited by non linearities of the system are not independent from each other, but harmonically related and dependent on the waveform shape of the resulting non sinusoidal oscillatory dynamics. Figure $15 \mathrm{C}$ shows the harmonicity-PAC plot associated to the single oscillatory dynamics of the Van der Pol oscillator (see Appendix A.2. Specifically, Figure $15 \mathrm{C}$ shows the 
evolution of the PAC (PLV) and harmonicity (TLI) metrics as the non linear parameter of the oscillator $\left(\mu / \omega_{0}\right)$ is increased from the sinusoidal oscillatory regime $\left(\mu / \omega_{0} \approx 0\right.$, see panels $\mathrm{A}$ and $\mathrm{F}$ in Figure 15$)$ up to a high non sinusoidal regime $\left(\mu / \omega_{0} \approx 4.77\right.$, see panels $\mathrm{D}$ and $\mathrm{K}$ in Figure 15$)$. In Figure 15 , the sinusoidal oscillatory regime $\left(\mu / \omega_{0} \approx 0\right)$ shown in panels $\mathrm{A}$ and $\mathrm{F}$, becomes evident by the single spectral component constituting the corresponding power spectra (see panels B and G), and by the phase portraits shown in Figures $B .6 \mathrm{~A}$ and B.6B. On the other hand, the non sinusoidal oscillatory regime $\left(\mu / \omega_{0} \approx 4.77\right)$ shown in panels $\mathrm{D}$ and $\mathrm{K}$, becomes evident by the harmonic spectral components constituting the corresponding power spectra (see panels E and L), and by the phase portraits shown in Figures B.6C,D. In Figures 15A,B,C,D,E, the dynamics of the Van der Pol oscillator was computed by configuring the intrinsic noise of type AWGN applied only on the equation of $\dot{x}_{2}\left(g_{1}=0\right.$ and $g_{2}=0.5$ in Eq. A.14. In this scenario, as the non sinusoidal oscillatory regime emerges, the harmonicity metric (TLI) allows for a clear identification of the harmonic nature of the PAC pattern. Figure $15 \mathrm{H}$ shows the behavior of the PAC pattern in the case when AWGN is being applied on the equations of both $\dot{x}_{1}$ and $\dot{x}_{2}$ (i.e. $g_{1}=g_{2}=0.5 \mathrm{in}$ Eq. A.14). On the other hand, Figure $15 \mathrm{H}$ shows that the harmonicity of the PAC intensity increases up to a given value of the non linear paremeter of the oscillator $\left(\mu / \omega_{0}\right)$, after which subsequent increments of $\mu / \omega_{0}$ produce a monotonic decrease of the harmonicity and keeping the PAC intensity unchanged. Figure 15 shows that the single oscillatory dynamics of the Van der Pol oscillator in presence of AWGN can elicit several PAC patterns depending on the value of $\mu / \omega_{0}$ : no PAC (Figures 15F,G), harmonic PAC (Figures 15[,J) and non harmonic PAC (Figures $15 \mathrm{~K}, \mathrm{~L}$ ). The results show in Figure $15 \mathrm{H}$ were computed using an epoch of $10 \mathrm{sec}$. which corresponds to approx. 50 cycles of the slowest oscillation at $f_{L F} \approx 4.7$ for $\mu / \omega_{0} \approx 4.8$. Importantly, it was found that these results holds even in the case of using an epoch length of $1.5 \mathrm{sec}$. ( $\approx 7$ cycles of the slowest oscillation), which is one order of magnitude shorter than that involved in the computation of Figure $15 \mathrm{H}$. Moreover, we found that the results presented in Figure 15 hold for the dynamics of the Van der Pol oscillator simulated with intrinsic noise of the type non-additive white Gaussian noise (NAWGN). The Harmonicity-PAC plots computed for the simulated dynamics of the Van der Pol oscillator with NAWGN intrinsic noise are shown in Figure B.7 of Appendix B.3. In addition, we verified that these results also hold when PAC is assessed using different metrics (e.g. PLV, KLMI), hence, discarding the possibility of artifacts associated to a particular metric (compare panels A vs. B and C vs. D shown in Figure B.7 of Appendix B.3. These results suggest that the presence of intrinsic noise (AWGN or NAWGN) can change the period of the single oscillatory dynamics in almost a cycle-by-cycle manner significantly reducing the harmonic content in its power spectrum. This evidence supports the conclusion that 'true' and 'spurious' concepts applied to the CFC patterns are not intrinsically linked to the harmonic content of the underlying oscillatory dynamics. More specifically, the high harmonic content observed in a given oscillatory dynamics is neither sufficient nor necessary condition to interpret the associated CFC pattern as 'spurious' or epiphenomenal 
829 (i.e. a CFC pattern not representing a true interaction between two coupled 830 oscillatory dynamics with independent fundamental frequencies). For instance, 831 a single oscillatory dynamics characterized by a non constant oscillation pe${ }_{832}$ riod can produce 'spurious' $\mathrm{CFC}$ with low harmonic content (i.e. non harmonic 833 CFC). This type of oscillatory dynamics is commonly observed in oscillators 834 undergoing a chaotic regime or non linear oscillators under the effect of intrinsic 835 noise (Figure 15H). On the other hand, in Sections 3.3 and 3.4 we shall present 836 results supporting the hypothesis that two coupled oscillatory dynamics with ${ }_{837}$ independent fundamental frequencies can elicit 'true' CFC with high harmonic 838 content via rectification mechanisms. 

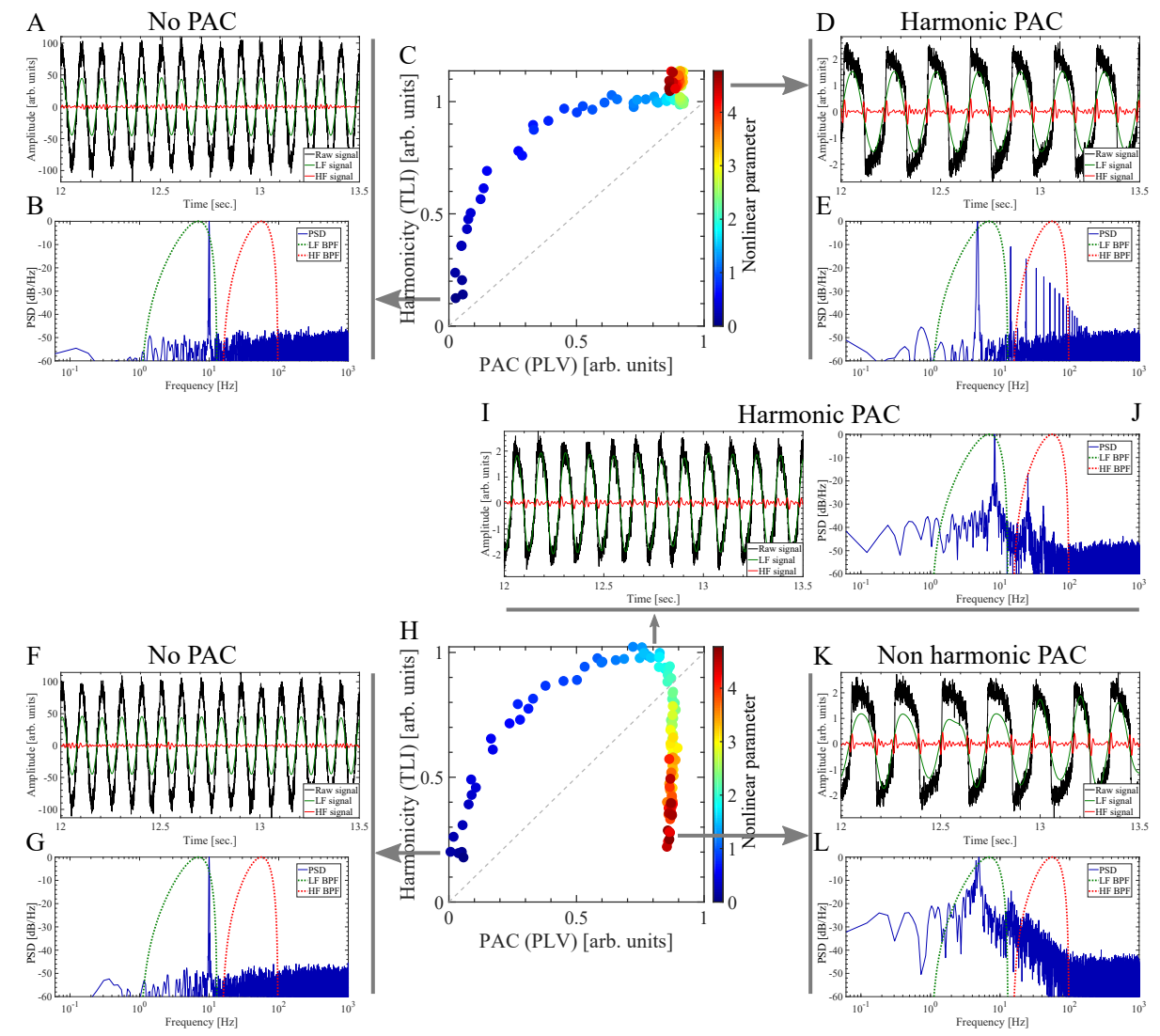

Figure 15: Harmonicity-PAC plot computed for the simulated dynamics of the Van der Pol oscillator with intrinsic noise of type additive white Gaussian noise (AWGN). Note that a single non sinusoidal oscillatory dynamics can produce both harmonic (panels D and E, I and J) and non harmonic (panels K and L) PAC patterns. The Van der Pol oscillatory dynamics (solid black line) shown in panels A, D, F, I and K were simulated as described in Appendix A.2 using the following hyperpameter values: sampling rate $f_{s}=2000 \mathrm{~Hz}, \omega_{0}=2 \pi f_{0}, f_{0}=10$ $\mathrm{Hz}, W_{p}=0, F_{e}=0$, initial conditions $x(0)=2, \dot{x}(0)=1$. To compute the harmonicity-PAC plots shown in panels $\mathrm{C}$ and $\mathrm{H}$, the parameter $\mu$ controlling the oscillator nonlinearity was increased from the sinusoidal oscillatory regime $\left(\mu / \omega_{0} \approx 0\right.$, see panels $\left.\mathrm{A}, \mathrm{B}, \mathrm{F}, \mathrm{G}\right)$ up to a high non sinusoidal regime $\left(\mu / \omega_{0} \approx 4.77\right.$, see panels $\left.\mathrm{D}, \mathrm{E}, \mathrm{I}, \mathrm{J}, \mathrm{K}, \mathrm{L}\right)$. In panels $\mathrm{C}$ and $\mathrm{H}$, the pseudocolor scale represents the $\mu / \omega_{0}$ values ranging from $\approx 0$ (blue) to $\approx 4.8$ (red). In panels A, B, C, D and E, the dynamics of the Van der Pol oscillator was simulated using intrinsic noise of type AWGN applied only on the equation of $\dot{x}_{2}\left(g_{1}=0\right.$ and $g_{2}=0.5$ in Eq. A.14. In panels F, G, H, I, J, K and L, the dynamics was simulated by applying the intrinsic noise of type AWGN on the equations of both $\dot{x}_{1}$ and $\dot{x}_{2}$ (i.e. $g_{1}=g_{2}=0.5$ in Eq. A.14. Therefore, in this case the intrinsic noise components (AWGN) in Eq. A.14 result $\eta_{1} \approx \mathcal{N}(0,0.5)$ and $\eta_{2} \approx \mathcal{N}(0,0.5)$. Extrinsic noise $\eta(t)$ was added as shown in Eq. A.14 In this case the noise level corresponds to the 10 percent of the maximum amplitude of the dynamics $x_{1}$ in Eq. A.14, scaling the standard deviation $\sigma$ of the additive white Gaussian noise $(\mathrm{AWGN}) \eta \approx \mathcal{N}(0, \sigma)$. We computed the Van der Pol dynamics for 20 sec. time interval and then the first $10 \mathrm{sec}$. of the time series were discarded to remove the transient period of the numerical simulation. The power spectra (solid blue line in graphs B, E, G, J and L) were computed unsing a $10 \mathrm{sec}$. epoch from the synthetic dynamics shown in the corresponding graphs (solid black line in graphs A, D, F, I and K). In graphs B, E, G, J and L, the power responses (i.e. square magnitude) of the BPF used to compute the LF and HF signals are shown as dotted green and red lines, respectively. To obtain all the band-pass filtered signals shown in this figure we use the BPF as descriped in Appendix A.5 In all the cases shown in this figure, the bandwidth of the BPF for the LF (LF BPF) and HF (HF BPF) components were set at $B w_{L F}=12 \mathrm{~Hz}$ centered at $7 \mathrm{~Hz}$ and $B w_{H F}=82.65 \mathrm{~Hz}$ centered at $56.5 \mathrm{~Hz}$, respectively. In graphs $\mathrm{A}, \mathrm{D}, \mathrm{F}, \mathrm{I}$ and $\mathrm{K}$, the resulting band-pass filtered LF and HF signals are shown as solid green and solid red lines, respectively. The harmonicity metric (TLI) was computed as it was described in Section 2.4 The PAC metric $\left(P L V_{P A C}\right)$ was computed using Eq. 4 with the configuration given by Eq. A.20 and $M=N=1$. 
It was found that the dependence of the harmonicity of the CFC pattern on the intrinsic noise (AWGN or NAWGN) is not exclusive of PAC but occurs in several CFC types. Figures $16 \mathrm{C}$ and $16 \mathrm{H}$ show this effect in the case of AAC and FFC patterns, respectively. To compute the Figure $16 \mathrm{C}$ we set Eqs. A.12, A.13 and A.14 with $W_{p}=0, f_{e}=5.4 \mathrm{~Hz}$ and $f_{m}=1.33 \mathrm{~Hz}$. This configuration produces an amplitude-modulated dynamics due to the action of the external driving $F_{e}$. This configuration produces an amplitude-modulated dynamics due to the action of the amplitude-modulated external driving $F_{e}$ in which the sinusoidal component at $f_{m}=1.33 \mathrm{~Hz}$ modulates the amplitude of the oscillation at $f_{e}=5.4 \mathrm{~Hz}$ (dotted grey line in Figures $16 \mathrm{~A}$ and $16 \mathrm{D}$ ). As a consequence, the slow rhythm at $f_{m}=1.33 \mathrm{~Hz}$ effectively modulates the amplitude of the non sinusoidal oscillator dynamics (solid black line in Figures 16A and 16D). The amplitude-modulation of the resulting non sinusoidal dynamics becomes evident in the phase portraits shown in Figure B.8. Thus, two CFC patterns emerge from the resulting dynamics, (1) a PAC pattern in which the phase of the slow rhythm at $f_{m}=1.33 \mathrm{~Hz}$ amplitude modulates the fundamental component of the non sinusoidal oscillator dynamics, and (2) an AAC pattern in which the amplitude of the harmonic components follow the changes of the fundamental component amplitude. In the PAC pattern we have a 'true' interaction between two oscillatory dynamics, i.e. $f_{m}=1.33 \mathrm{~Hz}$ and $f_{e}=5.4 \mathrm{~Hz}$. On the other hand, the AAC pattern can be thought as a 'spurious' or epiphenomenal coupling since it involves dependent frequencies related by the waveform shape of the single oscillatory dynamics. Figure $16 \mathrm{C}$ shows that the AAC intensity increases up to a given value of the external driving amplitude $\left(A_{e}\right.$ in Eq. A.13), after which subsequent increments of $A_{e}$ produce a significant drop in the harmonicity of the 'spurious' AAC pattern. To compute the Figure $16 \mathrm{H}$ we set Eqs. A.12. A.13 and A.14 with $F_{e}=0, f_{0}=10 \mathrm{~Hz}, f_{p} \approx 1 \mathrm{~Hz}$. As a result, we obtain an frequency-modulated dynamics due to the action of the time variant parameter $W_{p}$. Specifically, the slow rhythm at $f_{p} \approx 1 \mathrm{~Hz}$ (dotted gray line in Figures $16 \mathrm{~F}$ and 16I) effectively modulates the fundamental frequency of the non sinusoidal oscillator dynamics (solid black line in Figures $16 \mathrm{~F}$ and $16 \mathrm{~F}$ ). As a consequence, two CFC patterns emerge from the resulting dynamics, (1) a PFC pattern in which the phase of the slow rhythm at $f_{p} \approx 1 \mathrm{~Hz}$ frequency modulates the fundamental component of the non sinusoidal oscillator dynamics, and (2) an FFC pattern in which the instantaneous frequency of the harmonic components follow the changes of the fundamental component frequency. In the PFC pattern we have a 'true' interaction between two oscillatory dynamics, one associated to the time variant parameter $W_{p}$ and the other to the intrinsic dynamics of the oscillator. On the other hand, the FFC pattern can be thought as a 'spurious' or epiphenomenal coupling since it involves dependent frequencies related by the waveform shape of the single oscillatory dynamics. Figure $16 \mathrm{H}$ shows that the FFC intensity increases up to a given value of the $W_{p}$ intensity (i.e. $A_{p}$ in Eq. A.12, after which subsequent increments of $A_{p}$ produce a significant drop in the harmonicity of the 'spurious' FFC pattern. 

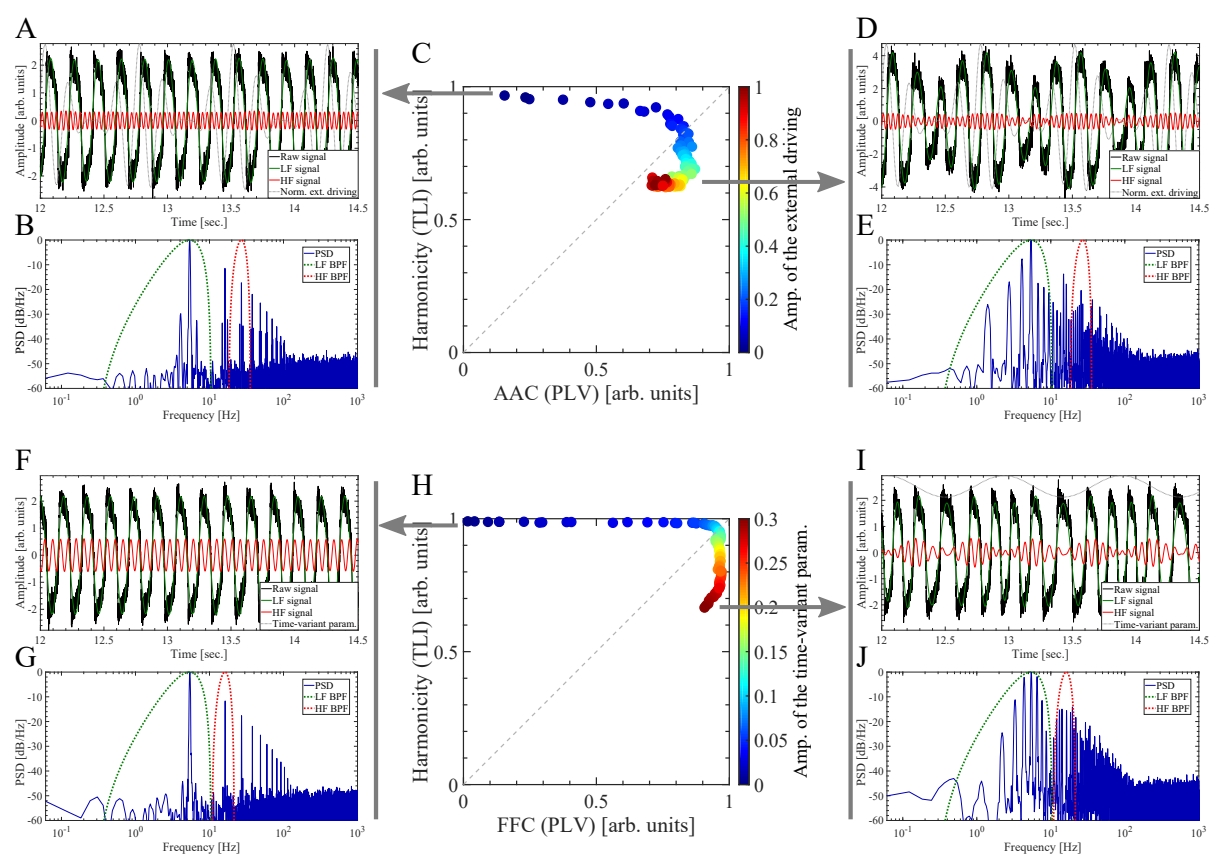

Figure 16: Harmonicity-AAC and Harmonicity-FFC plots computed for the simulated dynamics of the Van der Pol oscillator with intrinsic noise of type additive white Gaussian noise (AWGN). (A, B, C, D, E) To obtain the AAC pattern, the Van der Pol oscillatory dynamics (solid black line) shown in panels $\mathrm{A}$ and $\mathrm{D}$ were simulated as described in Appendix A.2 using Eqs. A.12 A.13 and A.14 with the following hyperpameter values: sampling rate $f_{s}=2000$ $\mathrm{Hz}, \omega_{0}=2 \pi f_{0}, f_{0}=10 \mathrm{~Hz}$, no parametric driving $W_{p}=0, f_{e}=5.4 \mathrm{~Hz}$ and $f_{m}=1.33$ $\mathrm{Hz}, A_{m}=1, c=1$ (i.e. DSB-C), maximum modulation depth $m=0$, initial conditions $x(0)=2, \dot{x}(0)=1$. To compute the harmonicity-AAC plot shown in panel $\mathrm{C}$, the external driving amplitude ( $A_{e}$ in Eq. A.13 was increased from $A_{e}=0$ (no external driving) up to $A_{e}=5 \times 10^{4}$. In panel $\mathrm{C}$, the pseudocolor scale represents the $A_{e} /\left(5 \times 10^{4}\right)$ values ranging from 0 (blue) to 1 (red). (F, G, H, I, J) To obtain the FFC pattern, the Van der Pol oscillatory dynamics (solid black line) shown in panels $\mathrm{F}$ and I were simulated as described in Appendix A.2 using Eqs. A.12 A.13 and A.14 with the following hyperpameter values: sampling rate $f_{s}=2000 \mathrm{~Hz}, \omega_{0}=2 \pi f_{0}, f_{0}=10 \mathrm{~Hz}$, no external driving $F_{e}=0, f_{p} \approx 1 \mathrm{~Hz}$. To compute the harmonicity-FFC plot shown in panel $\mathrm{H}$, the intensity of the time variant parameter $W_{p}$ ( $A_{p}$ in Eq. A.12 was increased from $A_{p}=0$ (no parametric driving) up to $A_{p} \approx 10$. In panel $\mathrm{H}$, the pseudocolor scale represents the $A_{p} / 34$ values ranging from 0 (blue) to 0.3 (red). For both panels $\mathrm{C}$ and $\mathrm{H}$, the dynamics of the Van der Pol oscillator was simulated using intrinsic noise of type AWGN applied only on the equation of $\dot{x}_{2}\left(g_{1}=0\right.$ and $g_{2}=0.5$ in Eq. A.14. Therefore, the intrinsic noise components (AWGN) in Eq. A.14 result $\eta_{1}=0$ and $\eta_{2} \approx \mathcal{N}(0,0.5)$. Extrinsic noise $\eta(t)$ was added as shown in Eq. A.14 In this case the noise level corresponds to the 10 percent of the maximum amplitude of the dynamics $x_{1}$ in Eq. A.14, scaling the standard deviation $\sigma$ of the additive white Gaussian noise (AWGN) $\eta \approx \mathcal{N}(0, \sigma)$. The Van der Pol dynamics was computed for 20 sec. time interval and then the first $10 \mathrm{sec}$. of the time series were discarded to remove the transient period of the numerical simulation. The power spectra (solid blue line in graphs B, E, G and J) were computed unsing a 10 sec. epoch from the synthetic dynamics shown in the corresponding graphs (solid black line in graphs A, D, F and I). In graphs B, E, G and J, the power responses (i.e. square magnitude) of the BPF used to compute the LF and HF signals are shown as dotted green and red lines, respectively. To obtain all the band-pass filtered signals shown in this figure we use the BPF as described in Appendix A.5 In computing panel C (AAC), the bandwidth of the $\mathrm{BPF}$ for the $\mathrm{LF}\left(\mathrm{LF} \mathrm{BPF}\right.$ ) and $\mathrm{HF}(\mathrm{HF} \mathrm{BPF})$ components were set at $B w_{L F} \approx 10.3 \mathrm{~Hz}$ centered at $5.4 \mathrm{~Hz}$ and $B w_{H F} \approx 17.6 \mathrm{~Hz}$ centered at $27 \mathrm{~Hz}$, respectively. In computing panel $\mathrm{H}$ (FFC), the bandwidth of the BPF for the LF (LF BPF) and HF (HF BPF) components were set at $B w_{L F} \approx 10.3 \mathrm{~Hz}$ centered at $5.44 \mathrm{~Hz}_{\mathrm{z}}$ and $B w_{H F} \approx 10.8 \mathrm{~Hz}$ centered at $16.2 \mathrm{~Hz}$, respectively. In graphs $\mathrm{A}, \mathrm{D}, \mathrm{F}$ and $\mathrm{I}$, the resulting band-pass filtered $\mathrm{LF}$ and $\mathrm{HF}$ signals are shown as solid green and solid red lines, respectively. The harmonicity metric (TLI) was computed as it was described in Section 2.4 For the panel C, the AAC metric $\left(P L V_{A A C}\right)$ was computed using Eq. 4 with the configuration given by Eq. A.21 and $M=N=1$. For the panel $\mathrm{H}$, the FFC metric $\left(P L V_{F F C}\right)$ was computed using Eq. 4 with the configuration given by Eq. A.24 and $M=N=1$. 


\subsection{Two coupled oscillatory dynamics characterized by independent frequencies}

In this section we present the results obtained with a 2 nd order parametric oscillator showing that two coupled oscillatory dynamics with independent fundamental frequencies can elicit 'true' CFC with high harmonic content via the rectification mechanism. The equations describing the dynamics of the parametric oscillator are detailed in Section 3.3 . Figure 17 shows the PFC patterns corresponding to the dynamics of the parametric oscillator generated by simultaneously applying an off-resonance external driving $F_{e}$ and a parametric driving $W_{p}$ tuned at the same frequency $f_{e}=f_{p}=f_{0} / 12 \approx 8.3 \mathrm{~Hz}$ and $\theta_{e}=0$ (see Eqs. A.16 and A.17), with $f_{0}$ being the natural resonance frequency of the undamped oscillator $(\mu=0$ in Eq. A.15). Figures $17 \mathrm{C}$ and $17 \mathrm{~J}$ show the harmonicity-PFC plots for the cases when AWGN is applied only on the equation of $\dot{x}_{2}\left(g_{1}=0\right.$ and $g_{2}=0.125$ in Eq. A.18) and on the equations of both $\dot{x}_{1}$ and $\dot{x}_{2}$ (i.e. $g_{1}=g_{2}=0.125$ in Eq. A.18), respectively. In the latter case, the intrinsic noise is capable to drive the resonator at its natural frequency $f_{0}$ increasing the harmonicity of the oscillatory dynamics for low $A_{p}$ values (see Figures 17 $\mathrm{H}, 17 \mathrm{I}$, and 17J). This harmonicity of the oscillatory dynamics for low $A_{p}$ values is not present when the parametric oscillator is configured with non harmonic frequencies (e.g. $f_{e}=f_{p}=f_{0} / 11.62 \approx 8.6 \mathrm{~Hz}$, see Figures $\mathrm{B} .10 \mathrm{H}$, B.10 and B.10J). Figures 17C 17J show that the harmonicity of the PFC pattern increases as the parametric driving intensity $A_{p}$ increases. In Figure 17 the almost sinusoidal oscillatory regime $\left(A_{p} \approx 0\right)$ shown in panel $\mathrm{A}$, becomes evident by the single spectral component constituting the corresponding power spectra (see panel B), and by the phase portrait shown in Figure B.9A. On the other hand, the non sinusoidal oscillatory regime $\left(A_{p} \approx 0.9\right)$ shown in panels $\mathrm{D}$ and $\mathrm{F}$, becomes evident by the harmonic spectral components constituting the corresponding power spectra (see panels E and G), and by the phase portraits shown in Figures B.6E and B.6F. In particular, panels D and E in Figure 17 show that the phase of the slow rhythm $\left(f_{L F}=f_{e}=f_{p} \approx 8.3 \mathrm{~Hz}\right)$ modulates both amplitude and frequency of the fast oscillation within the range $20 \mathrm{~Hz}$ $<f_{H F}<140 \mathrm{~Hz}$ (see Figure $4 \mathrm{~A}$ and $4 \mathrm{~B}$ ). We found that in the forced parametric oscillator, the fast oscillation constituting the oscillatory dynamics undergo a rectification process associated to the PAC pattern. That is, the amplitude of the HF component $\left(f_{H F}\right.$, solid red line in Figures $17 \mathrm{D}$ and $\left.17 \mathrm{~F}\right)$ goes to zero at some particular phase of the LF cycle $\left(f_{e}=f_{p}\right.$, solid green line in Figures 17D and $17 \mathrm{~F}$ ). This periodic rectification process produces that the HF component resets its phase relative to the LF component in each LF cycle. As a consequence, the waveform shape of the resulting oscillatory dynamics is almost the same in each LF cycle even when the slow and fast rhythms have independent frequencies. This repetitive waveform shape (Figures $17 \mathrm{D}$ and $17 \mathrm{~F}$ ) is characterized by a high harmonic content in its power spectrum (Figures $17 \mathrm{E}$ and $17 \mathrm{G}$ ) which accounts for the high harmonicity reported by the TLI metric for high $W_{p}$ values (Figures $17 \mathrm{C}$ and $17 \mathrm{~J}$ ). Importantly, we found that harmonic PFC patterns like those shown in Figures 17D and $17 \mathrm{~F}$ are elicited for high values of the parametric driving $W_{p}$ irrespective of the ratio of the time scales involved 
in the parametric oscillator, i.e. harmonic PFC patterns occurs for harmonic (Figure 17) or non harmonic frequency ratios $f_{e} / f_{0}$ with $f_{e}=f_{p}$ (Figure B.10 in Appendix B.4. We also verified that these results also holds when PFC is assessed using different metrics (e.g. PLV, KLMI), hence, discarding the possibility of an artefact associated to a particular metric (compare Figures B.10 and B.11 in Appendix B.4. These results support the hypothesis that the harmonicity of the PFC pattern shown in Figures 17D, 17F, B.10D, B.10F, B.11D and $\mathrm{B} .11 \mathrm{~F}$ are not related to a fine-tuning of the parameters $f_{e}, f_{p}$ and $f_{0}$ of the parametric oscillator, but to an emerging rectification mechanism associated to the co-occurrence of PAC and PFC patterns which produce the phase resetting of the modulated HF component in each LF cycle. 

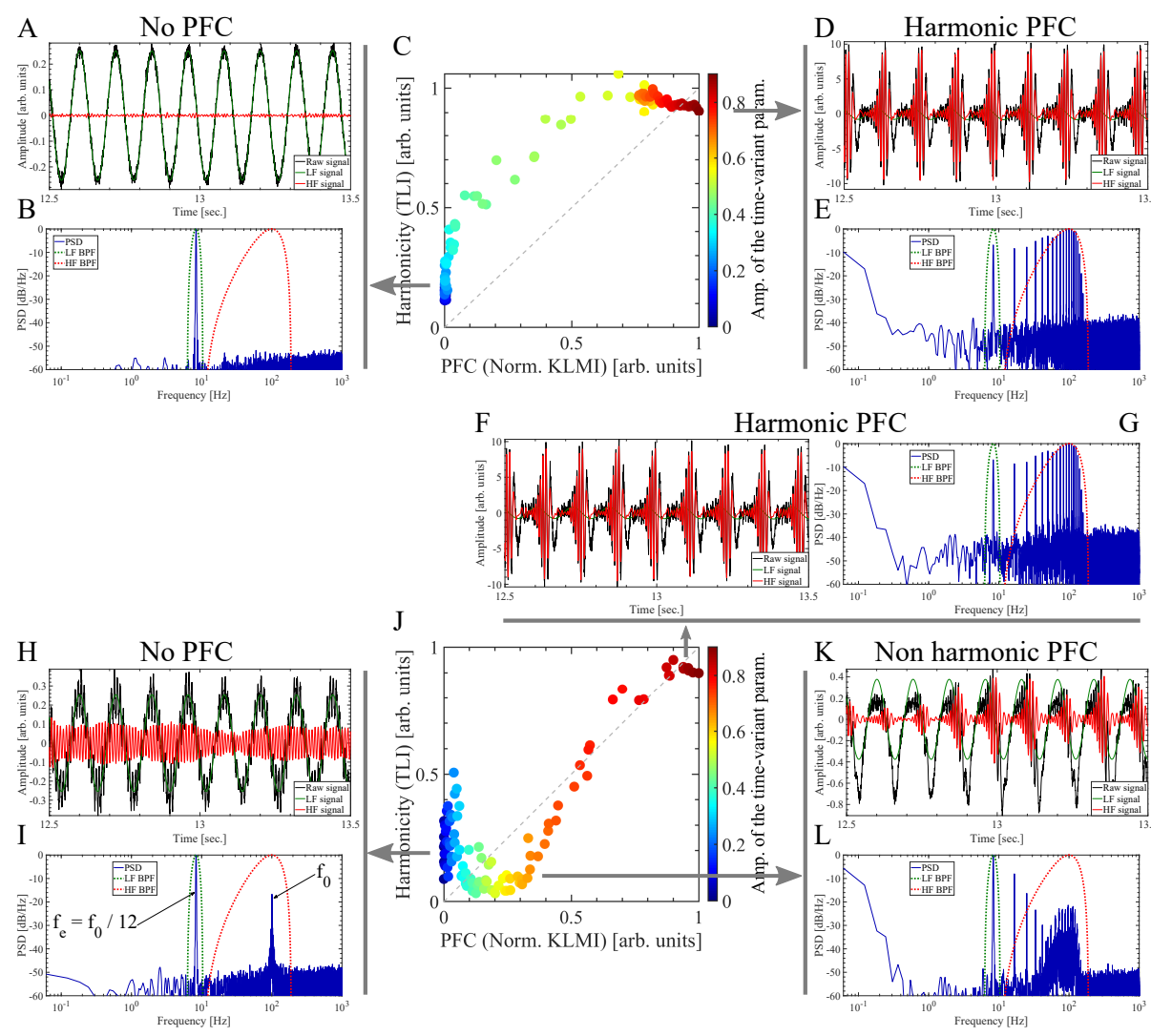

Figure 17: Harmonicity-PFC plot computed for the simulated dynamics of the 2nd order parametric oscillator with intrinsic noise of type additive white Gaussian noise (AWGN). Note that two oscillatory dynamics with independent frequencies can produce harmonic PFC patterns (panels D, E and F, G). The parametric oscillator dynamics (solid black line) shown in panels $\mathrm{A}, \mathrm{D}, \mathrm{H}, \mathrm{F}$ and $\mathrm{K}$ were simulated as described in Appendix A.3 by simultaneously applying an off-resonance external driving $F_{e}$ and a parametric driving $W_{p}$ tuned at the same frequency and using the following hyperpameter values: sampling rate $f_{s}=2000 \mathrm{~Hz}, \mu=200$, $\omega_{0}=2 \pi f_{0}, f_{0}=100 \mathrm{~Hz}, f_{p}=f_{e}=f_{0} / 12 \approx 8.3 \mathrm{~Hz}, \theta_{e}=0, A_{e}=1 \times 10^{5}$. To compute the harmonicity-PFC plots shown in panels $\mathrm{C}$ and $\mathrm{J}$, the parameter $A_{p}$ controlling the parametric driving intensity was increased from the sinusoidal oscillatory regime $\left(A_{p} \approx 0\right.$, see panels $\mathrm{A}$, $\mathrm{B}, \mathrm{H}, \mathrm{I})$ up to a high non sinusoidal regime $\left(A_{p} \approx 0.9\right.$, see panels $\left.\mathrm{D}, \mathrm{E}, \mathrm{F}, \mathrm{G}\right)$. In panels $\mathrm{C}$ and $\mathrm{J}$, the pseudocolor scale represents the $A_{p}$ values ranging from 0 (blue) to 0.9 (red). In panels $\mathrm{A}, \mathrm{B}, \mathrm{C}, \mathrm{D}$ and $\mathrm{E}$, the dynamics of the parametric oscillator was simulated using intrinsic noise of type AWGN applied only on the equation of $\dot{x}_{2}\left(g_{1}=0\right.$ and $g_{2}=0.125$ in Eq. A.18. In panels F, G, H, I, J, K and L, the dynamics was simulated by applying the intrinsic noise of type AWGN on the equations of both $\dot{x}_{1}$ and $\dot{x}_{2}$ (i.e. $g_{1}=g_{2}=0.125$ in Eq. A.18. Therefore, in this case the intrinsic noise components (AWGN) in Eq. A.18 result $\eta_{1} \approx \mathcal{N}(0,0.125)$ and $\eta_{2} \approx \mathcal{N}(0,0.125)$. Extrinsic noise $\eta(t)$ was added as shown in Eq. A.14 In this case the noise level corresponds to the 5 percent of the maximum amplitude of the dynamics $x_{1}$ in Eq. A.18, scaling the standard deviation $\sigma$ of the additive white Gaussian noise (AWGN) $\eta \approx \overline{\mathcal{N}}(0, \sigma)$. We computed the dynamics of the parametric oscillator for 20 sec. time interval and then the first $10 \mathrm{sec}$. of the time series were discarded to remove the transient period of the numerical simulation. The power spectra (solid blue line in graphs B, E, G, I and L) were computed unsing a $10 \mathrm{sec}$. epoch from the synthetic dynamics shown in the corresponding graphs (solid black line in graphs A, D, F, H and K). In graphs B, E, G, I and $\mathrm{L}$, the power responses (i.e. square magnitude) of the BPF used to compute the LF and HF signals are shown as dotted green and red lines, respectively. To obtain all the band-pass filtered signals shown in this figure we use th468PF as described in Appendix A.5 In all the cases shown in this figure, the bandwidth of the BPF for the LF (LF BPF) and HF (HF BPF) components were set at $B w_{L F} \approx 4.2 \mathrm{~Hz}$ centered at $f_{0} / 12 \approx 8.3 \mathrm{~Hz}$ and $B w_{H F} \approx 179 \mathrm{~Hz}$ centered at $f_{0}=100 \mathrm{~Hz}$, respectively. In graphs $\mathrm{A}, \mathrm{D}, \mathrm{F}, \mathrm{H}$ and $\mathrm{K}$, the resulting band-pass filtered LF and HF signals are shown as solid green and solid red lines, respectively. The harmonicity metric (TLI) was computed as it was described in Section 2.4 The PAC metric $\left(K L M I_{P F C}\right)$ was computed using Eqs. 6 and 7 with the configuration given by Eq. A.22 Note that the $K L M I_{P F C}$ was normalized with its maximum value in each plot. 


\subsection{Biologically plausible neural network model}

In this section we show that 'true' PAC patterns with high harmonic content (i.e, 'true' harmonic PAC) naturally emerge in the oscillatory dynamics of the biologically plausible neural network model shown in Figure 1. This model is considered as a canonical circuit for generating PAC [16], and it represents a network architecture that has been observed in a variety of sensory cortex areas in the form of a slow input stimuli (e.g. visual, auditory, olfactory) which entrain fast gamma oscillations underpinning local neural processing [13. In addition, the model shown in Figure 1 has been analyzed in the context of the parkinsonian basal ganglia-thalamocortical circuit under dopamine depletion in connection with both the mechanism of action of the deep-brain stimulation therapy 14 and the exaggerated PAC between beta-gamma frequency bands. The latter, putatively associated to the pathological mechanism of motor symptoms in Parkinson's disease [1, 33, 34.

In 1 we demonstrate that PAC phenomenon naturally emerges in mean-field models of biologically plausible networks, as a signature of specific bifurcation structures. In particular, for the model shown in Figure 1 we found that in the case of an oscillatory external driving without noise (i.e. $H_{i}=h_{i} \cos \left(\omega_{i} t+\phi_{i}\right)+d_{i}$ and $\eta_{i}=0$ for $I_{i}, i \in\{1,2\}$ in Eq. 11), the PAC patterns observed in the resulting dynamics were elicited by the periodic excitation/inhibition (PEI) of a network population producing intermittent fast oscillations (i.e. intermittent PAC). For a detailes discussion of the PEI mechanism associated to the model shown in Figure 1 the reader is referred to Section 3.1 and Appendix A of 1 . The threshold linear activation function $S\left(I_{i}\right)$ (Eq. 2 imposes certain conditions in the input space $\left(H_{1}, H_{2}\right)$ for the activation of the two populations constituting the architecture shown in Figure 1. As a consequence, when the amplitude of the inputs are high enough to activate the two populations, the intrinsic fast rhythm $(50 \mathrm{~Hz})$ coexist with the external slow driving $\left(\omega_{i} /(2 \pi)=3.33 \mathrm{~Hz}\right)$ in the resulting oscillatory dynamics. The fast rhythm cease if any of the two populations is deactivated. The locus in the $\left(H_{1}, H_{2}\right)$ space defined by the activation conditions does not depends on the temporal evolution of the inputs $H_{1}, H_{2}$ (See Figure 13 in Appendix A of [1]). As a result, the trajectory of a periodic driving dynamics $\left(H_{1}(t), H_{2}(t)\right)$ crosses the locus of the activation condition in the same phase of the slow driving period $\left(\omega_{i} /(2 \pi)=3.33 \mathrm{~Hz}\right)$. Thus, in the case of oscillatory inputs $H_{1}$ and/or $H_{2}$ capable to periodically activate and deactivate the populations of the intrinsic oscillator we obtain a PAC pattern associated to the intermittent occurrence of the fast rhythm phase locked to the slow external driving (i.e. PEI mechanism. See Figure 13 in Appendix A of [1]).

Importantly, due to the rectification process involved in the PEI mechanism in presence of threshold linear activation functions, the amplitude of the fast oscillation goes to zero at some particular phase of the slow cycle, hence, the fast oscillation resets its phase relative to the slow driving in each cycle (see Figure 18D). As a consequence, the waveform shape of the resulting oscillatory dynamics is almost the same in each slow cycle even when the slow and fast rhythms have independent frequencies. This repetitive waveform shape (Figure 
18D) is characterized by a high harmonic content in its power spectrum (Figure 18 E) which accounts for the high harmonicity reported by the TLI metric for high driving amplitude values (Figure $18 \mathrm{C}$ ). We found that increasing levels of intrinsic noise $\eta_{i}$ (see Section 2.1) applied on the model constituted by threshold linear activation function $S\left(I_{i}\right)$ produce a drop in both the harmonicity and the intensity of the PAC as shown in Figures $18 \mathrm{C}, 18 \mathrm{H}$ and $18 \mathrm{M}$. That is, it seems that the harmonic content and the PAC intensity are intrinsically linked by the rectification mechanism associated to threshold linear activation functions.

We also investigate the characteristics of the PAC patterns observed in the oscillatory dynamics of the model shown in Figure 1 constituted by the infinitely differentiable softplus activation function defined in Eq. 3. It was found that in absence of noise, the PEI mechanism associated to softplus activation functions elicit PAC patterns with high harmonic content (i.e. harmonic PAC) in the resulting oscillatory dynamics (data not shown). However, in a more realistic scenario including a small level of intrinsic noise $\eta_{i}$ applied on the model constituted by softplus activation function $S_{c}\left(I_{i}\right)$, the harmonicity was significantly reduced and the PAC instensity was kept almost unchanged (see Figure B.12 in Appendix B.5. This result suggest that the harmonic content and the PAC intensity are not intrinsically coupled in presence of the softplus activation function and can be interpreted as follows. Due to the fact that $S_{c}\left(I_{i}\right)>0$, the amplitude of the intrinsic fast rhythm $(50 \mathrm{~Hz})$ is effectively modulated by the external driving $\left(\omega_{i} /(2 \pi)=3.33 \mathrm{~Hz}\right)$ but it does not becomes strictly zero at any phase of the slow rhythm, hence, the fast oscillation never resets its phase relative to the slow driving. Thus, the two oscillations with incommensurable frequencies $(50 \mathrm{~Hz}, 3.3 \mathrm{~Hz})$ coupled via the PEI mechanism in absence of phase reseting, produce an oscillatory dynamics similar to that shown in the right panels of Figure 3 (non harmonic PAC). 
bioRxiv preprint doi: https://doi.org/10.1101/2020.10.15.341800; this version posted November 1, 2020. The copyright holder for this preprint (which was not certified by peer review) is the author/funder, who has granted bioRxiv a license to display the preprint in perpetuity. It is made available under aCC-BY-NC-ND 4.0 International license.
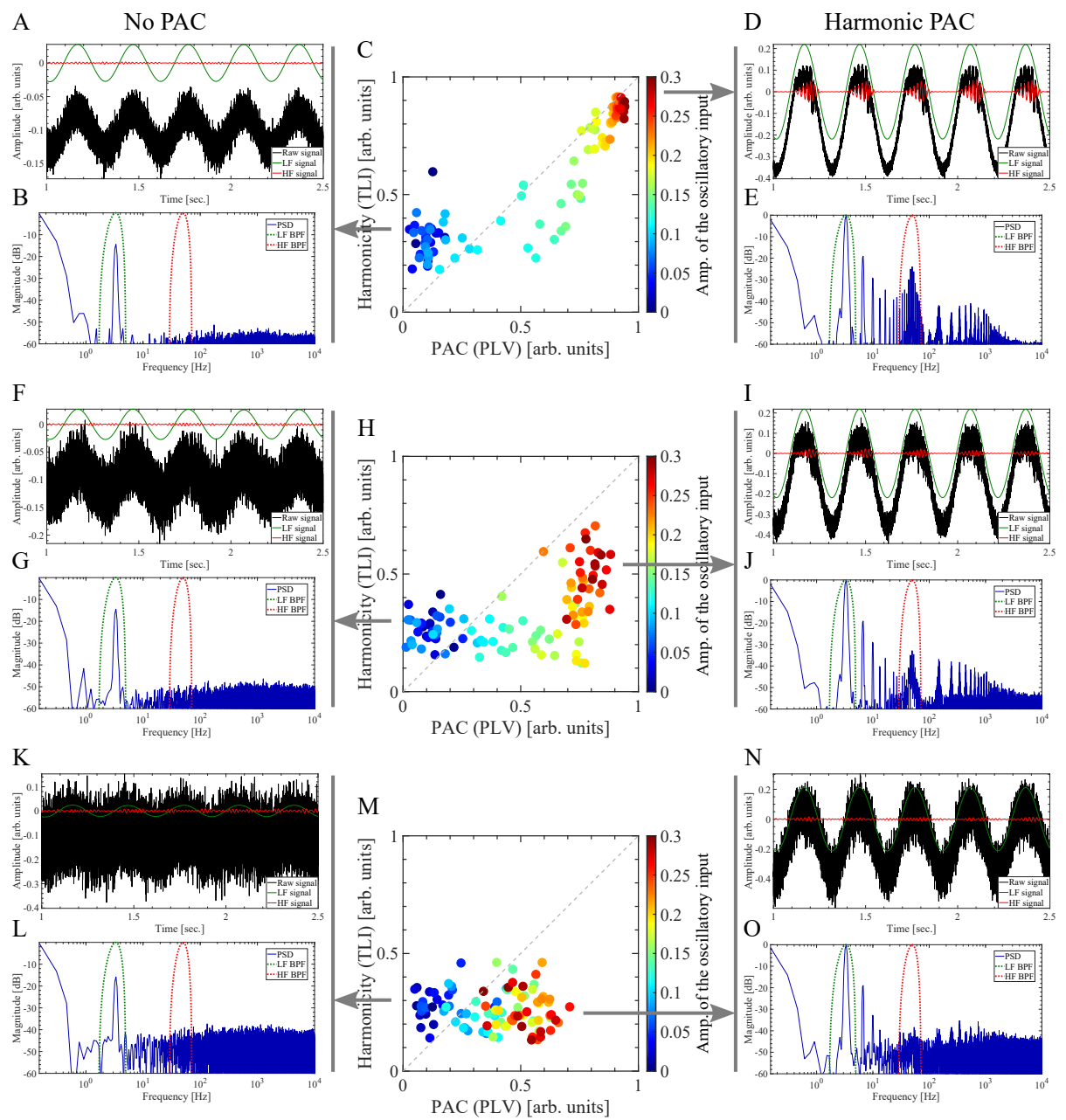

No PAC

Non harmonic PAC

Figure 18: Harmonicity-PAC plot computed for the simulated dynamics of the biologically plausible neural network model shown in Figure 1 using the threshold linear activation function $S\left(I_{i}\right)$ (Eq. 2). Note that two coupled oscillatory dynamics with independent fundamental frequencies can produce 'true' PAC patterns with high harmonic content via rectification mechanisms (panels D and E). The neural network dynamics (solid black line) shown in panels $\mathrm{A}, \mathrm{D}, \mathrm{F}, \mathrm{I}, \mathrm{K}$ and $\mathrm{N}$ were simulated as described in 2.1 using the configuration detailed in Table 1 resulting in an oscillatory dynamics in the gamma band $(50 \mathrm{~Hz})$. Besides, we use the following set of hyperpameter values: sampling rate $f_{s}=20 \mathrm{kHz}, H_{1}=0, H_{2}=A_{2} \cos \left(2 \pi f_{2} t\right)$ with $f_{2} \approx 3.3 \mathrm{~Hz}$. To compute the harmonicity-PAC plots shown in panels $\mathrm{C}, \mathrm{H}$ and $\mathrm{M}$, the parameter $A_{2}$ controlling the amplitude of the oscillatory input $H_{2}$ was increased from $A_{2}=0$ (see panels A, B, F, G, K, L) up to $A_{2}=0.3$ (see panels D, E, I, J, N, O). In panels C, H and $\mathrm{M}$, the pseudocolor scale represents the $A_{2}$ values ranging from $\approx 0$ (blue) to $\approx 0.3$ (red). The neural network dynamics was simulated using intrinsic noise $\eta_{i}$ of type AWGN to the node inputs $I_{i}$ (see Section 2.1). Panels $\mathrm{C}, \mathrm{H}$ and $\mathrm{M}$ were computed with a noise level of 5, 10 and 20 percent of the external input maximum amplitude $\left(A_{2}=0.3\right)$ scaling the standard deviation $\sigma_{i}$ of the additive white Gaussian noise $\eta_{i} \approx \mathcal{N}\left(0, \sigma_{i}\right)$, respectively. We computed theneural network dynamics for $10 \mathrm{sec}$. time interval and then the first $5 \mathrm{sec}$. of the time series were discarded to remove the transient period of the numerical simulation. The power spectra (solid blue line in graphs B, E, G, J, L and O) were computed unsing a 5 sec. epoch from the synthetic dynamics shown in the corresponding graphs (solid black line in graphs A, D, F, I, K and N). In graphs B, E, G, J, L and 19 , the power responses (i.e. square magnitude) of the BPF used to compute the LF and HF signals are shown as dotted green and red lines, respectively. To obtain all the band-pass filtered signals shown in this figure we use the BPF as described in Appendix A.5 In all the cases shown in this figure, the bandwidth of the BPF for the LF (LF BPF) and $\mathrm{HF}$ ( $\mathrm{HF} \mathrm{BPF}$ ) components were set at $B w_{L F} \approx 3.3 \mathrm{~Hz}$ centered at $\approx 3.3 \mathrm{~Hz}$ and $B w_{H F} \approx 43.2 \mathrm{~Hz}$ centered at $50 \mathrm{~Hz}$, respectively. In graphs $\mathrm{A}, \mathrm{D}, \mathrm{F}, \mathrm{I}, \mathrm{K}$ and $\mathrm{N}$, the resulting band-pass filtered LF and HF signals are shown as solid green and solid red lines, respectively. The harmonicity metric (TLI) was computed as it was described in Section 2.4 The PAC metric $\left(P L V_{P A C}\right)$ was computed using Eq. 4 with the configuration given by Eq. A.20 and $M=N=1$. 


\section{DISCUSSION}

In this work we provided an in-depth characterization of the Time Locked Index (TLI) as a novel tool aimed to efficiently quantify the harmonic content of noisy time series, and to assist the interpretation of CFC patterns observed in oscillatory dynamics of physical and biophysical systems. It was demonstrated that by operating in the time domain the TLI reliably assesses the degree of time-locking between the slow and fast rhythms, even in the case in which several (harmonic) spectral components are included within the bandwidth of the filter used to obtain the fast rhythm. In this aspect, the TLI measure outperforms the PLV and pairwise phase consistency metrics since the former is more robust against changes in the bandwidth or transition bands steepness of the BPF used to compute the HF component (see Figures 5, 8, 13 and 14 and related discussion).

We exploited the TLI metric together with other complementary signal processing tools to perform the harmonicity analysis on several types of CFC patterns using simulated and synthetic oscillatory dynamics under controlled levels of extrinsic (i.e. of observation) and intrinsic noise. To avoid the introduction of unnecessary timescales on the analyzed oscillatory dynamics, White Gaussian noise (AWGN) was used for this purpose. Since CFC is a rather ubiquitous phenomenon observed in a variety of physical systems, from physiological signals in the endocrine and cardiorespiratory systems, the neural activity of the human brain to the atmospheric variables, astronomical observations, earth seismic waves, nonlinear acoustics and stock market fluctuations (see [1] and references therein), our approach introduces a novel signal processing toolbox (and methodology) relevant to many physical and biophysical disciplines. CFC phenomenon observed in neural recordings has been proposed to be functionally involved in neuronal communication, memory formation and learning. In particular, experimental findings have shown that PAC and PPC patterns are important variants of CFC linked to physiological and pathological brain states like those observed in Parkinson's disease and epilepsy [1, 11, 31, 34. As it was discussed in Section 1 we recall that PPC is a signature related to the presence of harmonic spectral components in the underlying oscillatory dynamics. In this regard, the interpretation of the PAC patterns observed in local field potentials (LFP) recorded in humans and animal models remains challenging due to the fact that the brain activity is, in general, characterized by non sinusoidal oscillatory dynamics. The latter raises the question of whether PAC patterns are indicative of true interactions reflecting a mechanistic process between two independent neural oscillators, or whether it might be a more trivial consequence of spectral correlations due to the non sinusoidal waveform constituting the recorded time series [1, 20, 35. The apparent PAC that arises from non sinusoidal dynamics with high harmonic content has been hypothesized to be informative about the underlying neural processes [7, and it was experimentally demonstrated for interacting non linear acoustic oscillators in [36. However, the interpretation of the PAC phenomenon is completely different according to the mechanism that generates it. For instance, in [11 we 
demonstrated, through a harmonicity-PAC analysis using the TLI metric, that harmonic and non harmonic PAC patterns coexist during the seizure dynamics recorded with intracerebral macroelectrodes in epilepsy patients. We found that harmonic and non harmonic PAC patterns observed during the ictal activity can be interpreted as emerging features linked to the restrained and paroxysmal depolarizing shifts, which constitutes two essentially different neural mechanisms of seizure propagation. Importantly, the capability of the TLI metric to quantitatively distinguish the non harmonic PAC pattern, is clinically relevant since this specific pattern has been previously associated with the ictal core through the paroxysmal depolarizing shifts mechanism of seizure propagation.

The evidence discussed above highlights the relevance to unravel the complex interplay between spectral harmonicity and different types of CFC. Several approaches and controls have been previously proposed to address the true/spurious dichotomy in connection with PAC. In [37] it was argued that an increase in PAC intensity associated with a decrease in power of the modulating LF component would be an indication of the existence of 'true' coupling. Conversely, the presence of concomitant AAC and PAC patterns could be a proxy for 'spurious' PAC, in which the thigh correlation between the amplitude of the putative modulating LF and the modulated HF rhythms giving rise the AAC pattern, are produced by harmonically related spectral components constituting an underlying non sinusoidal oscillatory dynamics 38. Another approach suggested in [37] refers to the use of multimodal recordings (e.g. LFP, single and multi unit activity). Specifically, analysis of spike-triggered LFP recordings can be used to confirm that spike timing is clocked by the phase of ongoing HF component (e.g. gamma oscillations), hence, revealing that the modulated fast rhythm is not an HF harmonic of the slow modulating rhythm but associated to genuine HF oscillatory activity. Multi site recordings allow measures of inter area PAC in which slow and fast rhythms are extracted from time series recorded in different neural populations. Importantly, measures of inter area PAC in which the slow and fast rhythms are generated in distinct oscillators reduce concerns on spurious coupling [37, 39]. In this regard, it has been noted that one-to-one mapping between electrode measurement (i.e. time series) and neural source of oscillations (e.g. LFP) does not hold in real data, as there are multiple neural networks that generate fields measured by a single electrode [17, 40. Thus, the electrode time series is the result of a mix that could have very non sinusoidal waveform shape that is not present in any of the individual sources [17, 40]. Multichannel recordings in combination with tools for CFC source identification have been proposed as a way to disambiguates this issue 17, 40, 39.

Here we noted that in all these previous works it has been implicitly assumed that 'spurious' CFC patterns are intrinsically linked to an underlying non sinusoidal oscillatory dynamics characterized by a high harmonic content in its power spectrum. However, our results suggest that this assumption does not hold in realistic scenarios. In Velarde et al. 1] we analytically demonstrated that PAC phenomenon naturally emerges in mean-field models of biologically plausible networks, as a signature of specific bifurcation structures. Importantly, Velarde 
et al. 1 found that the mechanisms producing 'true' PAC (i.e. secondary Hopf bifurcation and PEI mechanism), in general elicit two coupled non sinusoidal oscillatory dynamics with independent fundamental frequencies. These results suggest that the resulting oscillatory dynamics underlying 'true' PAC is in general characterized by a high harmonic content in its power spectrum. In this work we quantitatively analyzed the role of the spectral harmonicity in different types of CFC patterns not restricted only to PAC and thus, providing a broader vision on this open issue in comparison to that addressed in previous reports. The results obtained using biologically plausible neural network models and more generic non linear and parametric oscillators reveal that harmonicity-CFC interplay is more complex than previously thought.

In line with the discussion given above about co-occurring AAC and PAC paterns [37, 38, we found that special care should be taken to interpret CFC patterns involving the same properties in the LF and HF frequency bands (e.g. PPC, AAC, FFC) since they might be epiphenomenal patterns elicited by a single non sinusoidal oscillatory dynamics constituted by harmonically related frequency components, in which the harmonic components within the HF band follows the changes of the fundamental frequency component in the LF band (see Section 3.2). However, in Sections 3.3 and 3.4 we show that concomitant PAC and PFC patterns were related to the presence of 'true' PFC with high harmonic content via the rectification mechanisms elicited by the PAC pattern. As a conclusion, the co-occurrence of multiple CFC patterns should not be taken as a straightforward indicator of spurious coupling per se. In Section 3.2 we show that a single oscillatory dynamics characterized by a non constant oscillation period can produce 'spurious' CFC with low harmonic content (i.e. non harmonic CFC). This type of oscillatory dynamics is commonly observed in oscillators undergoing a chaotic regime or non linear oscillators under the effect of intrinsic noise (Figure 15H). On the other hand, in Sections 3.3 and 3.4 we show that two coupled oscillatory dynamics with independent fundamental frequencies can elicit 'true' CFC with high harmonic content via rectification mechanisms (or other post-interaction nonlinear processing mechanisms). In Table 3 we resume the evidence supporting the conclusion that 'true' and 'spurious' concepts applied to the CFC patterns are not intrinsically linked to the harmonic content of the underlying oscillatory dynamics. Based on this results, we claim that the high harmonic content observed in a given oscillatory dynamics is neither sufficient nor necessary condition to interpret the associated CFC pattern as 'spurious' or epiphenomenal, i.e. not representing a true interaction between two coupled oscillatory dynamics with independent fundamental frequencies. 
Table 3: Summary of the harmonic and nonharmonic cross-frequency couplings observed in simulated and experimental oscillatory dynamics.

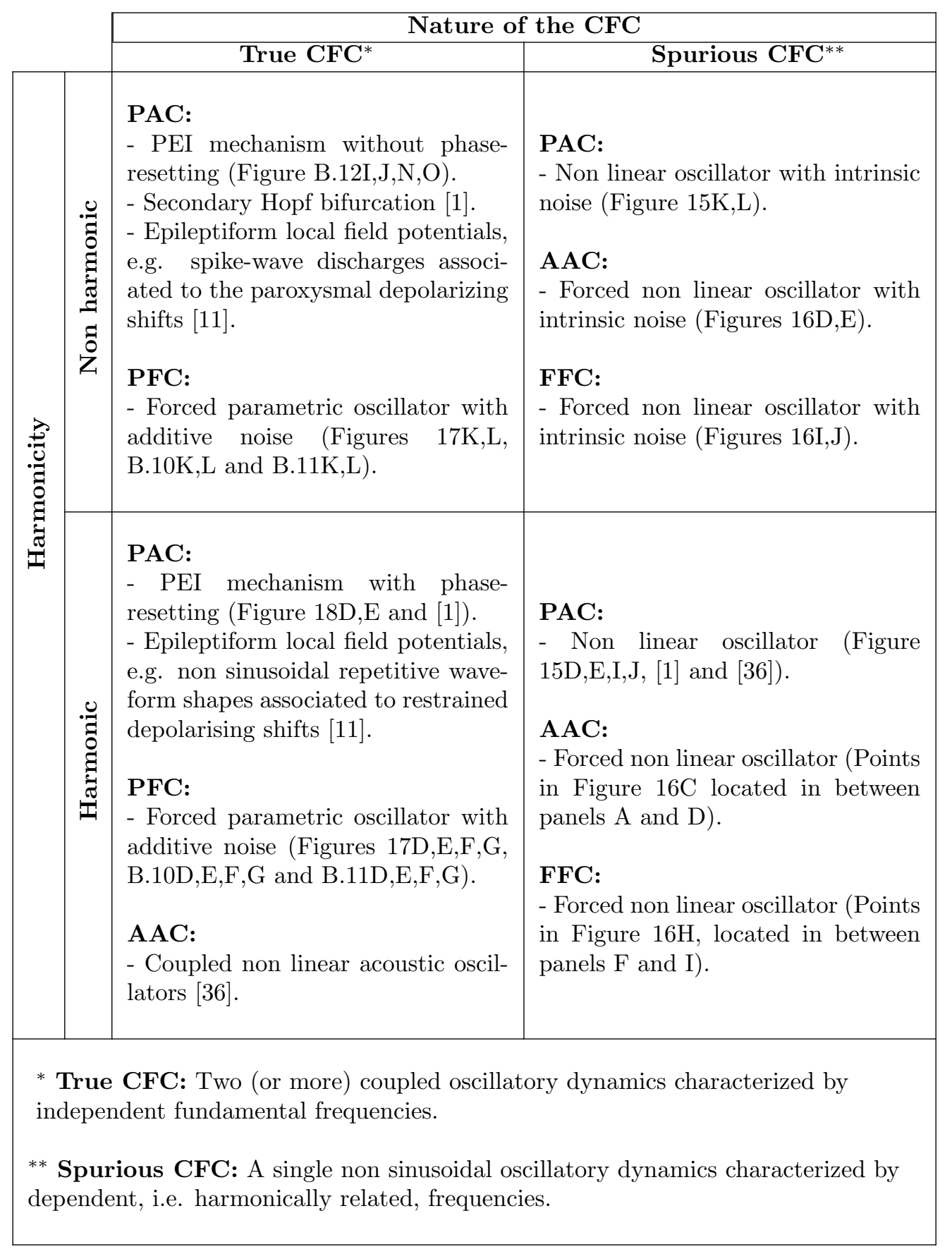




\section{CONCLUSION}

We found that harmonic and non harmonic patterns associated to a variety of CFC types (e.g. PAC, PFC) naturally emerges in the dynamics characterizing biologically plausible neural network models and more generic non linear and parametric oscillators. Substantial evidence was presented supporting the conclusion that 'true' and 'spurious' concepts applied to the CFC patterns are not intrinsically linked to the harmonic content of the underlying oscillatory dynamics. More specifically, the high harmonic content observed in a given oscillatory dynamics is neither sufficient nor necessary condition to interpret the associated CFC pattern as 'spurious' or epiphenomenal, i.e. not representing a true interaction between two coupled oscillatory dynamics with independent fundamental frequencies. In addition, the proposed signal processing techniques provide an extension of the traditional analytic toolkit used to quantify and interpret CFC patterns observed in oscillatory dynamics elicited by physical and biophysical systems. There is mounting evidence suggesting that the combination of multimodal recordings, specialized signal processing techniques and theoretical modeling is becoming a required step to completely understand CFC patterns observed in oscillatory rich dynamics of physical and biophysical systems.

\section{ACKNOWLEDGEMENTS}

This work was partially supported by UNCuyo-SeCTyP 2019 (80020180100576UN, 80020180100653UN) and Consejo Nacional de Investigaciones Científicas y Técnicas (CONICET) PIP 11220130100 256, Argentina.

\section{REFERENCES}

[1] O. M. Velarde, E. Urdapilleta, G. Mato, D. Dellavale, Bifurcation structure determines different phase-amplitude coupling patterns in the activity of biologically plausible neural networks, NeuroImage 202 (2019) 116031. doi : $10.1016 / j$.neuroimage.2019.116031.

[2] R. T. Canolty, E. Edwards, S. S. Dalal, M. Soltani, S. S. Nagarajan, H. E. Kirsch, M. S. Berger, N. M. Barbaro, R. T. Knight, High Gamma Power Is Phase-Locked to Theta Oscillations in Human Neocortex, Science 313 (5793) (2006) 1626-1628. doi:10.1126/science.1128115.

[3] P. Tass, M. G. Rosenblum, J. Weule, J. Kurths, A. Pikovsky, J. Volkmann, A. Schnitzler, H.-J. Freund, Detection of $n: m$ Phase Locking from Noisy Data: Application to Magnetoencephalography, Physical Review Letters 81 (0) (1998) 3291-3294. doi:10.1103/PhysRevLett.81.3291.

[4] W. Penny, E. Duzel, K. Miller, J. Ojemann, Testing for nested oscillation, Journal of Neuroscience Methods 174 (1) (2008) 50 - 61. doi:10.1016/j. jneumeth.2008.06.035. 
[5] M. X. Cohen, Assessing transient cross-frequency coupling in EEG data, Journal of Neuroscience Methods 168 (2) (2008) 494 - 499. doi:10.1016/ j.jneumeth.2007.10.012.

[6] A. B. L. Tort, R. Komorowski, H. Eichenbaum, N. Kopell, Measuring Phase-Amplitude Coupling Between Neuronal Oscillations of Different Frequencies, Journal of Neurophysiology 104 (2) (2010) 1195-1210. doi:10.1152/jn.00106.2010.

[7] S. R. Cole, B. Voytek, Brain Oscillations and the Importance of Waveform Shape, Trends in Cognitive Sciences (Regul. Ed.) 21 (2) (2017) 137-149. doi:10.1016/j.tics.2016.12.008

[8] N. Schaworonkow, V. V. Nikulin, Spatial neuronal synchronization and the waveform of oscillations: Implications for EEG and MEG, PLoS Computational Biology 15 (5) (2019) e1007055. doi:10.1371/journal.pcbi. 1007055

[9] S. R. Cole, R. van der Meij, E. J. Peterson, C. de Hemptinne, P. A. Starr, B. Voytek, Nonsinusoidal Beta Oscillations Reflect Cortical Pathophysiology in Parkinson's Disease, The Journal of Neuroscience 37 (18) (2017) 4830-4840. doi:10.1523/JNEUROSCI .2208-16.2017.

[10] N. Jackson, S. R. Cole, B. Voytek, N. C. Swann, Characteristics of Waveform Shape in Parkinson's Disease Detected With Scalp Electroencephalography, eNeuro 6 (3) (2019) 1-11. doi:10.1523/ENEURO.0151-19.2019.

[11] D. Dellavale, E. Urdapilleta, N. Cámpora, O. Velarde, S. Kochen, G. Mato, Two types of ictal phase-amplitude couplings in epilepsy patients revealed by spectral harmonicity of intracerebral EEG recordings, Clinical Neurophysiology 131 (8) (2020) 1866-1885. doi:10.1016/j.clinph.2020.04. 160.

[12] D. Haufler, D. Paré, Detection of Multiway Gamma Coordination Reveals How Frequency Mixing Shapes Neural Dynamics, Neuron 101 (4) (2019) 603-614. doi:10.1016/j.neuron.2018.12.028.

[13] A. Hyafil, A.-L. Giraud, L. Fontolan, B. Gutkin, Neural Cross-Frequency Coupling: Connecting Architectures, Mechanisms, and Functions, Trends

11. in Neurosciences 38 (11) (2015) 725-740. doi:10.1016/j.tins.2015.09. 001.

[14] O. M. Velarde, G. Mato, D. Dellavale, Mechanisms for pattern specificity of deep-brain stimulation in Parkinson's disease, PLOS ONE 12 (8) (2017) 1-30. doi:10.1371/journal.pone.0182884

[15] H. R. Wilson, J. D. Cowan, Excitatory and inhibitory interactions in localized populations of model neurons, Biophysical Journal 12 (1) (1972) 1-24. doi:10.1016/S0006-3495(72)86068-5. 
[16] A. C. E. Onslow, M. W. Jones, R. Bogacz, A Canonical Circuit for Generating Phase-Amplitude Coupling, PLOS ONE 9 (8) (2014) 1-15. doi:10.1371/journal.pone.0102591.

[17] M. X. Cohen, Analyzing neural time series data : theory and practice, MIT Press, 2014.

[18] J. van Driel, R. Cox, M. X. Cohen, Phase-clustering bias in phase-amplitude cross-frequency coupling and its removal, Journal of Neun roscience Methods 254 (2015) 60 - 72. doi:10.1016/j.jneumeth.2015. 07.014

[19] J. I. Berman, J. McDaniel, S. Liu, L. Cornew, W. Gaetz, T. P. Roberts, J. C. Edgar, Variable bandwidth filtering for improved sensitivity of cross1 frequency coupling metrics, Brain Connectivity 2(3) (2012) 155-63. doi: 10.1089/brain.2012.0085.

[20] D. Lozano-Soldevilla, N. ter Huurne, R. Oostenveld, Neuronal Oscillations with Non-sinusoidal Morphology Produce Spurious Phase-to-Amplitude Coupling and Directionality, Frontiers in Computational Neuroscience 10 (2016) 87. doi:10.3389/fncom.2016.00087.

[21] A. P. Vaz, R. B. Yaffe, J. H. Wittig, S. K. Inati, K. A. Zaghloul, Dual origins of measured phase-amplitude coupling reveal distinct neural mechanisms underlying episodic memory in the human cortex, NeuroImage 148 (2017) 148-159. doi:10.1016/j.neuroimage.2017.01.001.

[22] C. K. Kovach, A Biased Look at Phase Locking: Brief Critical Review and Proposed Remedy, IEEE TRANSACTIONS ON SIGNAL PROCESSING 65 (2017) 4468-4480. doi:10.1109/TSP.2017.2711517.

[23] M. Vinck, M. van Wingerden, T. Womelsdorf, P. Fries, C. M. A. Pennartz, The pairwise phase consistency: A bias-free measure of rhythmic

a neuronal synchronization, NeuroImage 51 (2010) 112-122. doi:10.1016/ j.neuroimage.2010.01.073

[24] S. Aydore, D. Pantazis, R. M. Leahy, A note on the phase locking value and its properties, NeuroImage 74 (2013) 231-244. doi:10.1016/j. neuroimage.2013.02.008.

[25] J. P. Lachaux, E. Rodriguez, J. Martinerie, , F. J. Varela, Measuring phase synchrony in brain signals, Human Brain Mapping 8 (4) (1999)

19 194-208. doi:10.1002/(SICI) 1097-0193(1999) 8:4<194: :AID-HBM4>3. $0 . \mathrm{CO} ; 2-\mathrm{C}$.

[26] M. Chehelcheraghi, C. van Leeuwen, E. Steur, C. Nakatani, A neural mass model of cross frequency coupling, PLOS ONE 12 (4) (2017) e0173776. doi:10.1371/journal.pone.0173776. 
[27] D. Vakman, On the analytic signal, the Teager-Kaiser energy algorithm, and other methods for defining amplitude and frequency, IEEE Transactions on Signal Processing 44 (4) (1996) 791 - 797. doi:10.1109/78. 492532

[28] G. F., S. A.Y., K. J.W., P. T., F. I., Cross-Modulated Amplitudes and Frequencies Characterize Interacting Components in Complex Systems, Physical Review Letters 102 (9) (2009) 098701. doi:10.1103/PhysRevLett. 102.098701 .

[29] A. V. Oppenheim, R. W. Schafer, J. R. Buck, Discrete-Time Signal Processing, 2nd Edition, Prentice Hall, 1998.

[30] A. B. L. Tort, M. A. Kramer, C. Thorn, D. J. Gibson, Y. Kubota, A. M. Graybiel, N. J. Kopell, Dynamic cross-frequency couplings of local field potential oscillations in rat striatum and hippocampus during performance of a T-maze task, Proceedings of the National Academy of Sciences 105 (51) (2008) 20517-20522. doi:10.1073/pnas.0810524105.

[31] B. J. He, J. M. Zempel, A. Z. Snyder, M. E. Raichle, The temporal structures and functional significance of scale-free brain activity, Neuron 66(3) (2010) 353-69. doi:10.1016/j.neuron.2010.04.020.

[32] A. Widmann, E. Schröger, Filter Effects and Filter Artifacts in the Analysis of Electrophysiological Data, Frontiers in Psychology 3:233 (2012) 1-5. doi:10.3389/fpsyg.2012.00233.

[33] J. López-Azcárate, M. Tainta, M. C. Rodríguez-Oroz, M. Valencia, R. González, J. Guridi, J. Iriarte, J. A. Obeso, J. Artieda, M. Alegre, Coupling between Beta and High-Frequency Activity in the $\mathrm{Hu}-$ man Subthalamic Nucleus May Be a Pathophysiological Mechanism in Parkinson's Disease, Journal of Neuroscience 30 (19) (2010) 6667-6677. doi:10.1523/JNEUROSCI.5459-09.2010.

[34] C. de Hemptinne, E. S. Ryapolova-Webb, E. L. Air, P. A. Garcia, K. J. Miller, J. G. Ojemann, J. L. Ostrem, N. B. Galifianakis, P. A. Starr, Exaggerated phase-amplitude coupling in the primary motor cortex in Parkinson disease, Proceedings of the National Academy of Sciences 110 (12) (2013) 4780-4785. doi:10.1073/pnas. 1214546110.

[35] J. Aru, J. Aru, V. Priesemann, M. Wibral, L. Lana, G. Pipa, W. Singer, R. Vicente, Untangling Cross-Frequency Coupling in Neuroscience, Current Opinion in Neurobiology 31 (2015) 51-61. doi:10.1016/j.conb.2014.08. 002 .

[36] D. Dellavale, J. M. Rosselló, Cross-frequency couplings in non-sinusoidal dynamics of interacting oscillators: Acoustic estimation of the radial position and spatial stability of nonlinear oscillating bubbles, Ultrasonics Sonochemistry 51 (2019) 424-438. doi:10.1016/j.ultsonch.2018.07.026. 
[37] O. Jensen, E. Spaak, H. Park, Discriminating Valid from Spurious Indices of Phase-Amplitude Coupling, eNeuro 3 (6) (2016) 1-8. doi:10.1523/ ENEURO.0334-16.2016

[38] J. Giehl, N. Noury, M. Siegel, Dissociating harmonic and non-harmonic phase-amplitude coupling in the human brain, bioRxivdoi:10.1101/2020. 08.11 .246298

[39] F. Siebenhühner, S. H. Wang, G. Arnulfo, A. Lampinen, L. Nobili, J. M. Palva, S. Palva, Genuine cross-frequency coupling networks in human resting-state electrophysiological recordings, PLoS Biology 18 (5) (2020) e3000685. doi:10.1371/journal.pbio.3000685.

[40] M. X. Cohen, Multivariate cross-frequency coupling via generalized eigendecomposition, eLife 6 (2017) e21792. doi:10.7554/eLife.21792.

[41] P. E. Kloeden, E. Platen, Numerical solution of stochastic differential equations, 2nd Edition, Springer, 1995.

[42] S. Haykin, Communication Systems, 4th Edition, John Wiley \& Sons, 2000.

[43] A. Delorme, S. Makeig, EEGLAB: an open source toolbox for analysis of single-trial EEG dynamics including independent component analysis, Journal of Neuroscience Methods 134 (2004) 9-21. doi:doi.org/10.1016/ j.jneumeth.2003.10.009. 


\section{Appendix A. Supplementary methods}

$$
\text { AM type }= \begin{cases}\text { Double-SideBand with Carrier (DSB-C), } & \forall c \geq 1 \\ \text { Double-SideBand Reduced-Carrier (DSB-RC), } & \forall 1>c>0 \\ \text { Double-SideBand Suppressed-Carrier (DSB-SC), } & \forall c=0\end{cases}
$$

\section{Appendix A.1. Synthetic signals}

Synthetic dynamics associated to the analysis of various PAC and PPC patterns were computed as follows,

$$
x(t)=\mathcal{E}(t)\left(z_{D S B}(t)+a(t)+z_{h}(t)+z_{H F}(t)\right)+\eta(t)
$$

In Eq. A.1. $t \in \mathbb{Z}$ is the discrete time index, $z_{D S B}(t)$ is the amplitude modulated (double side band) signal with a sinusoidal carrier of frequency $f_{H F}, a(t)$ is the modulating signal, $z_{h}(t)$ is a sum of harmonic oscillations of the fundamental frequency $f_{L F}, z_{H F}(t)$ is a sinusoidal component with frequency $f_{H F}, \eta(t)$ represent extrinsic (i.e. of observation) additive white Gaussian noise (AWGN). The amplitude envelope of the entire time series $\mathcal{E}(t)$ was included to emulate CFC and harmonicity transients in the synthetic dynamics and it was defined in terms of the sigmoid function,

$$
\begin{aligned}
& \mathcal{E}(t)=\mathcal{S}(t)+\mathcal{S}(-t) \\
& \mathcal{S}(t)=\frac{1}{1+e^{-\alpha(t-\beta)}},
\end{aligned}
$$

where $\alpha$ and $\beta$ are parameters controlling the edge steepness and the time shift of the time series envelope, respectively. For synthetic oscillatory dynamics in permanent regime with no transients we use $\mathcal{E}(t)=1$. The amplitude modulated signal $z_{D S B}(t)$ was defined as,

$$
z_{D S B}(t)=\left(\left(a(t)+\eta_{m}(t)\right)(1-m)+c A_{m}(1+m)\right) \sin \left(2 \pi f_{H F} t+\phi_{c}\right)
$$

In Eq. A.4 $a(t)$ defines the shape of the amplitude envelope of the sinusoidal carrier with frequency $f_{H F}, A_{m}$ is the maximum value of the modulating $a(t)$, $\eta_{m}(t)$ is additive white Gaussian noise (AWGN) intrinsic to the modulation process, $m$ define the modulation depth ( $m=0$ imply maximum modulation depth and $m=1$ for no modulation) and $c$ is the carrier factor controlling the type of amplitude modulation (AM),

For the sake of consistency with modulation depth $(m)$ and carrier factor $(c)$ parameters in Eq. A.4 the modulating signal $a(t)$ must satisfy the condition $\min (a(t))=-\max (a(t))$. We explored two types of waveform shapes for the periodic modulating signals $a(t)$. The sinusoidal modulating signal was defined as,

$$
a(t)=A_{m} \sin \left(2 \pi f_{L F} t+\phi_{m}\right)
$$


On the other hand, the periodic Gaussian modulating was defined as,

$$
\begin{aligned}
& a(t)=g\left(t \bmod \frac{T_{L F}}{2}\right) ; T_{L F}=\frac{1}{f_{L F}} \\
& g(t)=A_{m}\left(2 e^{-\frac{t^{2}}{2 \sigma^{2}}}-1\right)
\end{aligned}
$$

In Eq. A.8 $g(t)$ define a single period with the shape of a Gaussian probability density function with standard deviation $\sigma$. In Eqs. A.7. $g(t)$ is repeated with a period of $T_{L F}$ samples to obtain a periodic modulating signal $a(t)$ with a Gaussian waveform shape.

The $z_{h}(t)$ and $z_{H F}(t)$ signals were defined as follows,

$$
\begin{aligned}
z_{h}(t) & =\sum_{k=1}^{N_{h}} A_{k} \sin \left(k 2 \pi f_{L F} t+\phi_{k}\right) \\
z_{H F}(t) & =A_{H F} \sin \left(2 \pi f_{H F} t+\phi_{H F}\right)
\end{aligned}
$$

Appendix A.2. Van der Pol oscillator

The Van der Pol oscillator is a non linear and time invariant system whose dynamics is defined by the following differential equation,

$$
\ddot{x}-\mu\left(1-x^{2}\right) \dot{x}+\left(\omega_{0}+W_{p}\right)^{2} x=F_{e},
$$

where the over-dot represents time derivative, $\mu$ is a scalar parameter controlling the nonlinearity, $\omega_{0}=2 \pi f_{0}$ is the angular frequency of oscillation when $\mu=0$, $W_{p}=0$ and $F_{e}=0$. The time variant parameter $W_{p}$ and the external driving $F_{e}$ were defined as,

$$
\begin{aligned}
W_{p} & =A_{p} \cos \left(2 \pi f_{p} t\right) \\
A_{p} & =2 \pi F_{p} \\
F_{e} & =A_{e} \cos \left(2 \pi f_{e} t+\theta_{e}\right) \\
A_{e} & =A_{m} \cos \left(2 \pi f_{m} t\right)(1-m)+c A_{m}(1+m)
\end{aligned}
$$

${ }_{41}$ where $F_{p}$ and $f_{p}$ have units of $\mathrm{Hz}, F_{e}$ is defined as an amplitude-modulated ex-

${ }_{42}$ ternal driving with frequency $f_{e}$ and constant phase $\theta_{e}$, being $f_{m}$ the frequency

43 of the sinusoidal modulating, $A_{m}$ the maximum value of the modulating, $m$

44 the modulation depth and $c$ the carrier factor controlling the type of ampli-

45 tude modulation (see Eq. A.5). In presence of noise, the dynamics of the Van

46 der Pol oscillator is described by the following system of stochastic differential

47 equations,

$$
\left\{\begin{array}{l}
\dot{x}_{1}=x_{2}+g_{1} \eta_{1} \\
\dot{x}_{2}=\mu\left(1-x_{1}^{2}\right) x_{2}-\left(\omega_{0}+W_{p}\right)^{2} x_{1}+F_{e}+g_{2} \eta_{2} \\
x=x_{1}+\eta
\end{array}\right.
$$

48 In Eq. A.14, $\eta_{1}$ and $\eta_{2}$ are independent and identically distributed random vari49 ables representing intrinsic noise, and $\eta$ represent extrinsic (i.e. of observation) 
noise. For the simulations computed with the Eq. A.14, we use independent and normally distributed random variables for both intrinsic $\left(\eta_{1}, \eta_{2}\right)$ and extrinsic $(\eta)$ noise (i.e. white Gaussian noise). Therefore, the intrinsic noise components in Eq. A.14 result $\eta_{1} \approx \mathcal{N}\left(0, g_{1}\right)$ and $\eta_{2} \approx \mathcal{N}\left(0, g_{2}\right)$, where $g_{1}$ and $g_{2}$ represent the standard deviation of the zero-mean normal distribution $\mathcal{N}$. Unless otherwise specified, we use Additive White Gaussian Noise (AWGN), that is, the parameters defining the noise intensity $g_{1}$ and $g_{2}$ do not depend on the state variables $x_{1}$ and $x_{2}$. In the case of $g_{1}=g_{2}=0$, Eqs. A.14 and A.11 are equivalent. In addition, we fixed $f_{0}=10 \mathrm{~Hz}$ and $\theta_{e}=0$. Regarding the numerical integration of the stochastic differential equation A.14 we use an explicit solver based on the Euler-Heun method [41].

\section{Appendix A.3. Parametric oscillator}

To analyse the PFC patterns we use a linear and time variant system based on a 2 nd order parametric oscillator whose dynamics is defined by the following differential equation,

$$
\ddot{x}+\mu \dot{x}+\omega_{0}^{2}\left(1+W_{p}\right) x=F_{e},
$$

where the over-dot represents time derivative, $\mu$ is the parameter defining the intensity of the dissipative term, $\omega_{0}=2 \pi f_{0}$ is the angular frequency of oscillation when $\mu=0, W_{p}=0$ and $F_{e}=0$. The time variant parameter $W_{p}$ and the external driving $F_{e}$ were defined as,

$$
\begin{aligned}
W_{p} & =A_{p} \cos \left(2 \pi f_{p} t\right) \\
F_{e} & =A_{e} \cos \left(2 \pi f_{e} t+\theta_{e}\right),
\end{aligned}
$$

where $f_{p}$ and $f_{e}$ have units of $\mathrm{Hz}, \theta_{e}$ is a constant phase in rads., the parameters $A_{p}$ and $A_{e}$ defines the intensity of parametric and external driving, respectively. In presence of noise, the dynamics of the parametric oscillator is described by the following system of stochastic differential equations,

$$
\left\{\begin{array}{l}
\dot{x}_{1}=x_{2}+g_{1} \eta_{1} \\
\dot{x}_{2}=-\mu x_{2}-\omega_{0}^{2}\left(1+W_{p}\right) x_{1}+F_{e}+g_{2} \eta_{2} \\
x=x_{1}+\eta
\end{array}\right.
$$

In Eq. A.18, $\eta_{1}$ and $\eta_{2}$ are independent and identically distributed random variables representing intrinsic noise, and $\eta$ represent extrinsic (i.e. of observation) noise. For the simulations computed with the Eq. A.18, we use independent and normally distributed random variables for both intrinsic $\left(\eta_{1}, \eta_{2}\right)$ and extrinsic $(\eta)$ noise (i.e. white Gaussian noise). Therefore, the intrinsic noise components in Eq. A.18 result $\eta_{1} \approx \mathcal{N}\left(0, g_{1}\right)$ and $\eta_{2} \approx \mathcal{N}\left(0, g_{2}\right)$, where $g_{1}$ and $g_{2}$ represent the standard deviation of the zero-mean normal distribution $\mathcal{N}$. Unless otherwise specified, we use Additive White Gaussian Noise (AWGN), that is, the parameters defining the noise intensity $g_{1}$ and $g_{2}$ do not depend on the state variables $x_{1}$ and $x_{2}$. In the case of $g_{1}=g_{2}=0$, Eqs. A.18 and A.15 are equivalent. In addition, we fixed $f_{0}=100 \mathrm{~Hz}, \mu=200, f_{p}=f_{e}$. Regarding the numerical integration of the stochastic differential equation A.18 we use an explicit solver based on the Euler-Heun method [41]. 
Appendix A.4. Cross frequency coupling metrics

Eqs. A.19 to A.24 show the proper configuration of the $y_{H F}(t), \phi_{H F}(t)$ and $\phi_{L F}(t)$ time series to quantify PPC, PAC, AAC, PFC, AFC and FFC by means of the PLV, MVL and KLMI metrics using Eqs. 4 to 7.

$$
\begin{aligned}
& P P C\left\{\begin{array}{l}
\phi_{L F}=\arg \left(x_{L F}^{+}\right) \\
\phi_{H F}=\arg \left(x_{H F}^{+}\right)
\end{array}\right. \\
& P A C\left\{\begin{array}{l}
\phi_{L F}=\arg \left(x_{L F}^{+}\right) \\
\phi_{H F}=\arg \left(y_{H F}^{+}\right), y_{H F}=a_{H F}=\left|x_{H F}^{+}\right|
\end{array}\right. \\
& A A C\left\{\begin{array}{l}
\phi_{L F}=\arg \left(y_{L F}^{+}\right), y_{L F}=a_{L F}=\left|x_{L F}^{+}\right| \\
\phi_{H F}=\arg \left(y_{H F}^{+}\right), y_{H F}=a_{H F}=\left|x_{H F}^{+}\right|
\end{array}\right. \\
& P F C\left\{\begin{array}{l}
\phi_{L F}=\arg \left(x_{L F}^{+}\right) \\
\phi_{H F}=\arg \left(\Omega_{H F}^{+}\right), y_{H F}=\Omega_{H F}
\end{array}\right. \\
& A F C\left\{\begin{array}{l}
\phi_{L F}=\arg \left(y_{L F}^{+}\right), y_{L F}=a_{L F}=\left|x_{L F}^{+}\right| \\
\phi_{H F}=\arg \left(\Omega_{H F}^{+}\right), y_{H F}=\Omega_{H F}
\end{array}\right. \\
& F F C\left\{\begin{array}{l}
\phi_{L F}=\arg \left(\Omega_{L F}^{+}\right), y_{L F}=\Omega_{L F} \\
\phi_{H F}=\arg \left(\Omega_{H F}^{+}\right), y_{H F}=\Omega_{H F}
\end{array}\right.
\end{aligned}
$$

The time series configuration to assess PC using Eq. 8 is given by Eq. A.25.

$$
P C \quad\left\{\phi_{f}(t)=\arg \left(x_{f}^{+}(t)\right) ; f \in\{L F, H F\}\right.
$$

The $y_{H F}(t), \phi_{H F}(t)$ and $\phi_{L F}(t)$ time series required to assess the six types of CFC were computed using the Filter-Hilbert method (see Chapter 14 in [17]). In brief, the raw time series $x(t)$ was band-pass filtered around the frequency band of interest $f \in\{L F, H F\}$, then, the analytic signal $x_{f}^{+}(t)$ corresponding to the filtered time series $x_{f}(t)$ was computed in the frequency domain using the following equations [36, 42,

$$
\begin{aligned}
X_{f}(\omega) & =\mathcal{F}\left\{x_{f}(t)\right\} \\
X_{f}^{+}(\omega) & = \begin{cases}2 X_{f}(\omega), & \forall \omega>0 \\
X_{f}(0), & \forall \omega=0 \\
0, & \forall \omega<0\end{cases} \\
x_{f}^{+}(t) & =\mathcal{F}^{-1}\left\{X_{f}^{+}(\omega)\right\}=x_{f}(t)+i \hat{x_{f}}(t) \\
\hat{x_{f}}(t) & =\operatorname{Im}\left\{x_{f}^{+}(t)\right\}=\mathcal{H}\left\{x_{f}(t)\right\},
\end{aligned}
$$

In Eq. A.26, $t \in \mathbb{Z}$ is the discrete time index, $\omega$ is the non dimensional angular frequency (see Appendix A.6), $X_{f}(\omega)$ is the discrete Fourier transform, $i$ is the imaginary unit, $\mathcal{H}\{$.$\} denotes the Hilbert transform, \operatorname{Im}\left\{x_{f}^{+}(t)\right\}$ stands for the 
imaginary part of $x_{f}^{+}(t)$ and the operators $\mathcal{F}\{$.$\} and \mathcal{F}^{-1}\{$.$\} denote the discrete$ Fourier transformation and its inverse respectively, which were computed via the fast Fourier transform algorithm.

The amplitude envelope $a_{f}(t)$ and phase $\phi_{f}(t)$ time series for that particular frequency band $f \in\{L F, H F\}$ were obtained by computing the absolute value and argument of the analytic signal $x_{f}^{+}(t)$, respectively:

$$
\begin{aligned}
& \phi_{f}(t)=\arg \left(x_{f}^{+}(t)\right)=\arctan \left(\frac{\hat{x_{f}}(t)}{x_{f}(t)}\right)[\mathrm{rad} .] \\
& a_{f}(t)=\left|x_{f}^{+}(t)\right|
\end{aligned}
$$

In Eqs. A.22, A.23 and A.24, the instantaneous frequency time series $\Omega_{L F}(t)$ and $\Omega_{H F}(t)$ were computed following the procedures described in the Section 2.5 ,

\section{Appendix A.5. Band-pass filtering}

The band-pass filters (BPF) involved in the computation of $x_{f}(t)$ from the raw time series $x(t)$ were implemented in the frequency domain by multiplying the Fourier transform of the input signal by a Hann window and then, applying the inverse Fourier transform to get the band-pass filtered signal back in the time domain (i.e. circular convolution in the discrete time domain) [1, 11, 36]. Note that this filtering approach was used to effectively isolate the desired frequency bands (i.e. null-to-null bandwidth), which is not guaranteed when other linear filters are used (e.g. low order IIR filters) 31. We verified that our BPF implementation do not produce neither phase distortions nor significant artificial oscillations in the output signal capable to generate spurious CFC [32], showing a performance comparable to that of the FIR filters implemented in the EEGLAB (eegfilt function, data not shown) 43. In order to mitigate edge artifacts due to the transient response of the BPFs and the computation of the analytic signals, we implemented the time series reflection procedure described in 17. Briefly, time series are reversed in time, concatenated to both ends of the real-data time series, analyses were performed, and then, the reflected portion of the data were trimmed.

Appendix A.6. Fourier transform of the discrete time derivator

In this section we shall obtain the expression for the discrete Fourier transform of the discrete time derivator defined as,

$$
\frac{\phi_{f}(t)-\phi_{f}(t-1)}{T_{s}}
$$

where $T_{s}=1 / f_{s}$ is the sampling time interval corresponding to the sampling rate $f_{s}$. Taking into account the analysis and synthesis equations of the discrete 
time Fourier transform,

$$
\begin{aligned}
\mathcal{F}\left\{\phi_{f}(t)\right\}=\Phi_{f}(k) & =\sum_{t=0}^{N_{s}-1} \phi_{f}(t) e^{i \frac{2 \pi}{N_{s}} k t} \\
\phi_{f}(t) & =\frac{1}{N_{s}} \sum_{k=0}^{N_{s}-1} \Phi_{f}(k) e^{-i \frac{2 \pi}{N_{s}} k t},
\end{aligned}
$$

where $i$ is the imaginary unit, $t, k \in \mathbb{Z}$ are the discrete time and frequency indices, respectively and $N_{s}$ is the number of samples of the time series. Applying Eq. A.30 to Eq. A.29 and introducing the non dimensional angular frequency $\omega=k 2 \pi / N_{s}$, we obtain,

$$
\begin{aligned}
\mathcal{F}\left\{\frac{\phi_{f}(t)-\phi_{f}(t-1)}{T_{s}}\right\} & =f_{s} \Phi_{f}(\omega)\left(1-e^{-i \omega}\right) \\
& =f_{s} \Phi_{f}(\omega)(1-\cos (\omega)+i \sin (\omega))
\end{aligned}
$$

where we have applied the time shifting property of the Fourier transform (in this case for a time shift $t=-1$ ). The Eq. A.32 is the discrete Fourier transform of the discrete time derivator in Eq. A.29.

Let us now consider that the oversampling condition given by $f_{s} \gg f: f \in$ $\{L F, H F\}$ is satisfied. As a consequence, in the discrete frequency domain this condition implies $k \ll N_{s}$, or equivalently, $\omega \approx 0$. Under this condition, the Eq. A.33 can be well described by a first order approximation in the non dimensional angular frequency $\omega$ which can be written as,

$$
\mathcal{F}\left\{\frac{\phi_{f}(t)-\phi_{f}(t-1)}{T_{s}}\right\} \approx f_{s} \Phi_{f}(\omega)(1-1+i \omega)
$$

Appendix B. Supplementary results

\section{Appendix B.1. Bias of the TLI}

Figure B.1 shows that the TLI and $P L V_{P P C}$ metrics present a comparable bias when computed on non harmonically related oscillations. Figure B.1 shows that the bias of the TLI and $P L V_{P P C}$ metrics rapidly increases for epoch lengths shorter that $\approx 10$ cycles of the slow rhythm, being this bias rather independent of the noise level (AWGN) and the non harmonic ratio $\left(R=f_{H F} / f_{L F}\right)$ between the slow and fast oscillations. 
A

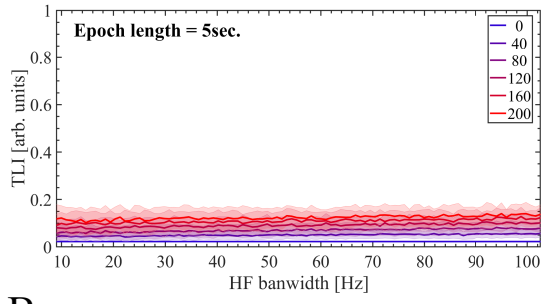

B

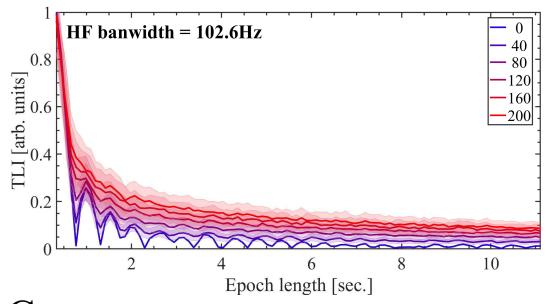

$\mathrm{C}$

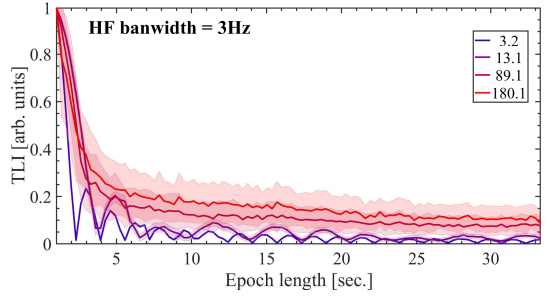

$\mathrm{D}$

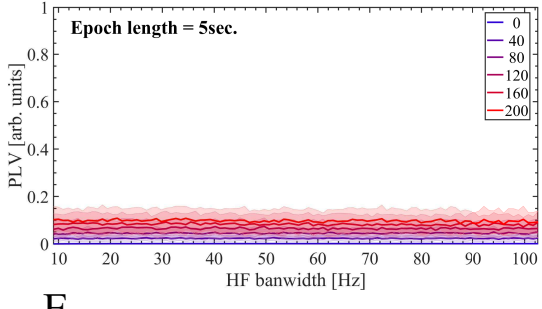

$\mathrm{E}$

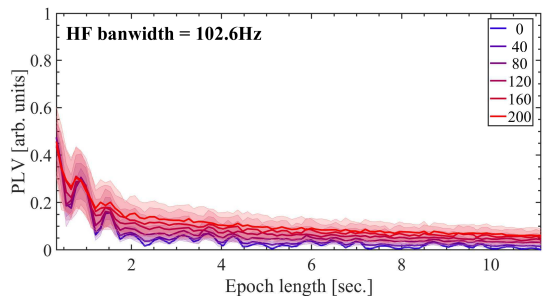

$\mathrm{F}$

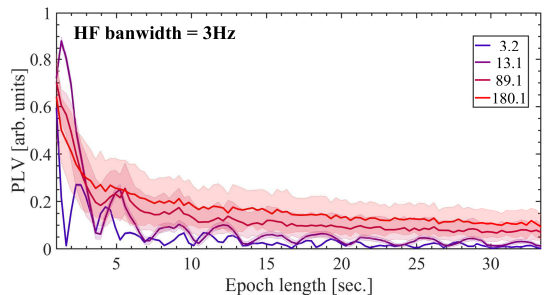

Figure B.1: The TLI and $P L V_{P P C}$ metrics present a comparable bias when computed on non harmonically related oscillations. In all the cases shown in this figure, we used a sampling rate of $f_{s}=2000 \mathrm{~Hz}$ and the bandwidth of the BPF for the LF component (LF BPF) was kept fixed at $B w_{L F}=f_{L F}$. We use the BPF as described in Appendix A.5 Besides, in all the cases shown in this figure the noise level is expressed as the percent of the amplitude of the $\mathrm{LF}$ component at $f_{L F} \mathrm{~Hz}$ scaling the standard deviation $\sigma$ of the additive white Gaussian noise $\mathcal{N}(0, \sigma)$. Panels $\mathrm{A}, \mathrm{B}, \mathrm{D}$ and $\mathrm{E}$, were computed using a synthetic dynamics similar to that used in Figure 5 but in this case it is constituted by two non harmonic oscillations at $f_{L F}=9 \mathrm{~Hz}$ and $f_{H F}=7.2 f_{L F}=64.8 \mathrm{~Hz}$. For panels A, B, D and E, the $P L V_{P P C}$ was computed using Eq. 4 with the configuration given by Eq. A.19 and $M=1, N=7$. (A, D) TLI and $P L V_{P P C}$ metrics as a function of the HF bandwidth $\left(B w_{H F}\right)$ corresponding to the filter HF BPF used to obtain the HF signal $\left(x_{H F}(t)\right)$, and taking the level AWGN as a parameter. The minimum and maximum $B w_{H F}$ values used to compute the graphs $\mathrm{B}$ and $\mathrm{E}$ were $9 \mathrm{~Hz}$ and $102.6 \mathrm{~Hz}$, respectively. To compute these graphs, the epoch length was kept unchanged in 5 sec. (B, E) TLI and $P L V_{P P C}$ metrics as a function of the epoch length and taking the level of additive white Gaussian noise (AWGN) as a parameter. To compute graphs $\mathrm{B}$ and $\mathrm{E}$, the bandwidth of the filter HF BPF was kept unchanged at $B w_{H F}=102.6 \mathrm{~Hz}$. Our implementation of the TLI algorithm (Section 2.4 requires at least 3 cycles of the low frequency oscillation $\left(f_{L F}=9 \mathrm{~Hz}\right)$, which determines the minimum epoch length shown in graphs $\mathrm{A}$ and $\mathrm{D}\left(3 / f_{L F} \approx 0.3 \mathrm{sec}\right.$.). The maximum epoch length used to compute graphs $\mathrm{A}$ and $\mathrm{D}$ was $100 / f_{L F} \approx 11.1 \mathrm{sec}$. (C, F) The TLI and $P L V_{P P C}$ metrics as a function of the epoch length and taking the non harmonic ratio $R=f_{H F} / f_{L F}$ as a parameter. Panels $\mathrm{C}$ and F were computed using a synthetic dynamics similar to that used in Figure 5 but in this case it is constituted by two non harmonic oscillations at $f_{L F}=3 \mathrm{~Hz}$ and $f_{H F}=R \times f_{L F}$ with $R=3.2,13.1,89.1,180.1$. To compute graphs $\mathrm{C}$ and $\mathrm{F}$, the bandwidth of the filter HF BPF was kept unchanged at $B w_{H F}=B w_{L F}=f_{L F}=3 \mathrm{~Hz}$. The noise level was set to 20 percent of the amplitude of the $\mathrm{LF}$ component at $f_{L F}=3 \mathrm{~Hz}$. The minimum and maximum epoch length shown in graphs $\mathrm{C}$ and $\mathrm{F}$ are $3 / f_{L F} \approx 1$ sec. and $100 / f_{L F} \approx 33.3$ sec., respectively. In all the panels, the solid lines represent the mean values and the shaded error bars correspond to the standard deviation of 100 realizations at each point. 
Appendix B.2. CFC time series and the bias produced by phase clustering

Figures B.2 and B.3 show the temporal evolution of the PAC $\left(P L V_{P A C}\right)$, harmonicity (TLI) and phase clustering $\left(P C_{L F}\right)$ metrics for synthetic dynamics presenting non PAC and a transient pattern of non harmonic PAC, respectively. Figures B.2 and B.3 should be compared with the results for a synthetic dynamics presenting a transient pattern of harmonic PAC (Figure 9). 
bioRxiv preprint doi: https://doi.org/10.1101/2020.10.15.341800; this version posted November 1, 2020. The copyright holder for this preprint (which was not certified by peer review) is the author/funder, who has granted bioRxiv a license to display the preprint in perpetuity. It is made available under aCC-BY-NC-ND 4.0 International license.

A

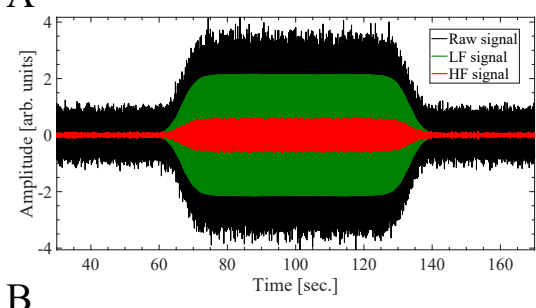

$\mathrm{B}$
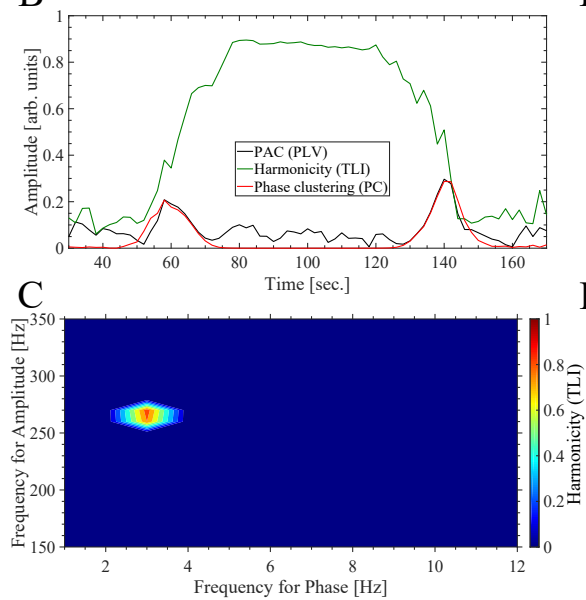

$\mathrm{D}$

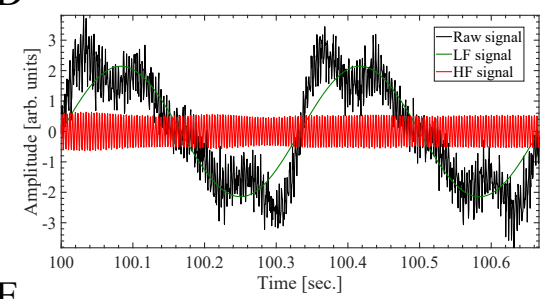

$\mathrm{E}$
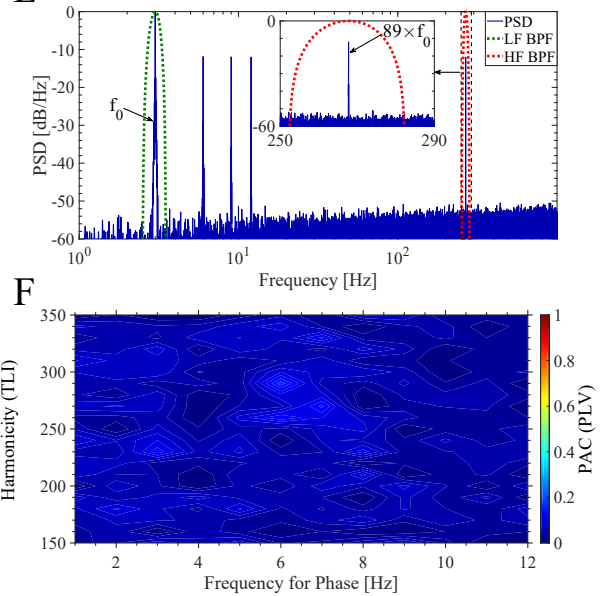

Figure B.2: Temporal evolution of the PAC $\left(P L V_{P A C}\right)$, harmonicity (TLI) and phase clustering $\left(P C_{L F}\right)$ metrics during a synthetic dynamics without PAC. To obtain all the band-pass filtered signals shown in this figure we use the BPF as described in Appendix A.5. (A) Synthetic dynamics (solid black line) together with the HF and LF signals shown as solid red and green lines, respectively. The dynamics (solid black line) was synthesized using Eqs. A.1 and A.4 with the following hyperpameter values: sampling rate $f_{s}=2000 \mathrm{~Hz}, c=1$ (i.e. DSB-C), zero modulation depth $m=0, \eta_{m}=0$, we used a sinusoidal modulating $a(t)$ with the fundamental frequency at $f_{0}=f_{L F}=3 \mathrm{~Hz}$ as given by Eq. A.6 with $A_{m}=1, z_{D B S}$ was set with $f_{H F}=89 \times f_{L F}=267 \mathrm{~Hz}, \phi_{c}=0, z_{H F}=0$, for $z_{h}$ we use $A_{1}=4, A_{k}=1 \forall 2 \leq k \leq 4$, $A_{k}=0 \forall k \geq 5$ and $\phi_{k}=0 \forall k$. The transient pattern was implemented through the time series envelope $\mathcal{E}(t)$ as defined in Eqs. A.2 and A.3 with $\alpha=0.5$ and $\beta$ equals to one third of the time series length. Extrinsic noise $\eta(t)$ was added as shown in Eq. A.1 In this case the noise level corresponds to the 10 percent of the maximum amplitude of the deterministic part of signal $x(t)$ (i.e first term of the right-hand member of the Eq. A.1), scaling the standard deviation $\sigma$ of the additive white Gaussian noise (AWGN) $\eta \approx \overline{\mathcal{N}}(0, \sigma)$. The LF (solid green line) and HF (solid red line) signals where obtained by filtering the raw signal (solid black line) with the band-pass filters whose power responses are shown as dotted green $\left(B w_{L F}=1 \mathrm{~Hz}\right)$ and red $\left(B w_{H F}=30 \mathrm{~Hz}\right)$ lines in graph E, respectively. (B) Time series showing the temporal evolution of the $P L V_{P A C}$, TLI and $P C_{L F}$ metrics. These time series were computed as described in Section 2.8 using the algorithm 2 summarized in Table 2 with a sliding window of $20 \mathrm{sec}$. in length, i.e. 60 cycles of the slowest oscillatory component at $f_{0}=f_{L F}=3 \mathrm{~Hz}$. (C) TLI harmonicity map computed as described in Section 2.7 using a 20 sec. epoch extracted from the center (Time $\approx 100 \mathrm{sec}$.) of the synthetic dynamics shown in panel A. In computing the map, all the TLI values below the significance threshold were set to zero (see Section 2.7). The pseudocolor scale represents the TLI values ranging from 0 (blue) to 1 (red). (D) Zoom showing two cycles of the synthetic dynamics (solid black line) together with the HF and LF signals shown as solid red and green lines, respectively. The two cycle epoch corresponds to the center (Time $\approx 100 \mathrm{sec}$.) of the synthetic dynamics shown in panel A. (E) Power spectrum (solid blue line) computed from the synthetic dynamics (solid black line in graph A). The power responses (i.e. square magnitude) of the BPF used to compute the LF and HF signals are shown as dotted green and red lines, respectively. (F) Comodulogram computed as described in Sectgon 2.7 computed from the same epoch used to obtain the harmonicity map (panel C). In computing the comodulogram, all the $\left|P L V_{P A C}\right|$ values below the significance threshold were set to zero (see Section 2.7). The pseudocolor scale represents the $\left|P L V_{P A C}\right|$ values ranging from 0 (blue) to 1 (red). The harmonicity map (panel C) and comodulogram (panel F) were computed using the same BPF (see Appendix A.5. 
A

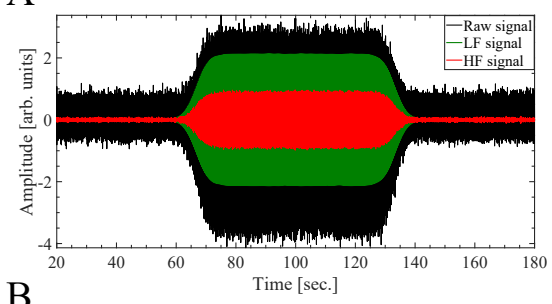

B
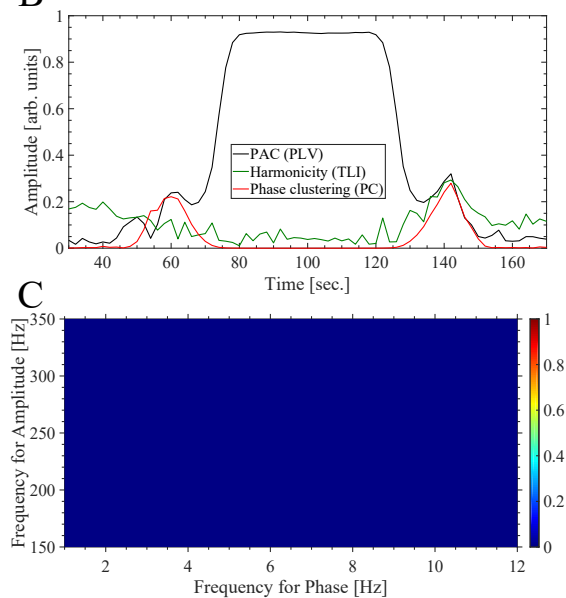

$\mathrm{D}$

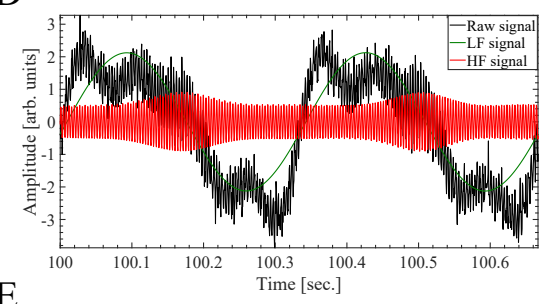

$\mathrm{E}$
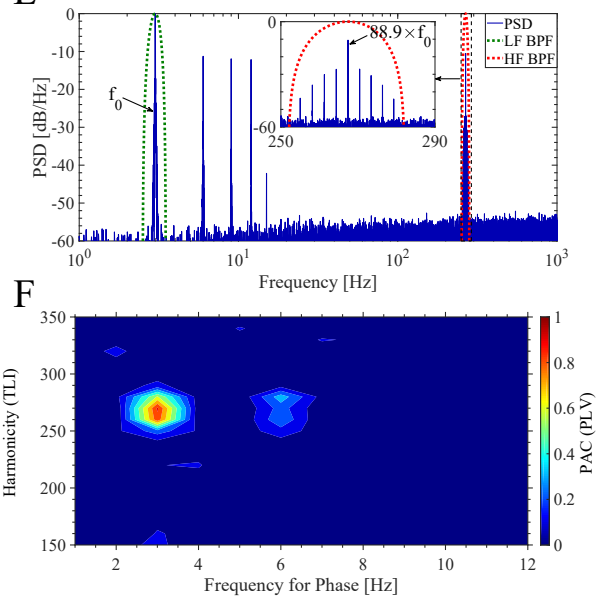

Figure B.3: Temporal evolution of the PAC ( $\left.P L V_{P A C}\right)$, harmonicity (TLI) and phase clustering $\left(P C_{L F}\right)$ metrics during a synthetic dynamics presenting a transient non harmonic PAC pattern. The synthetic dynamics was synthesized using the same parameter values than those used in Figure 9 except for the frequency of the carrier in the $z_{D B S}$ signal which in this case was set to $f_{H F}=88.9 \times f_{L F}=88.9 \times 3=266.7 \mathrm{~Hz}$. The $P L V_{P A C}$, TLI and $P C_{L F}$ metrics were computed using the same set of hyperparameter values than those used in Figure 9 The description of the panels is the same than that given in Figure 9

Figures 9 and 10 in the main text show that the presence of phase clustering $\left(P C_{L F}\right)$ produces a bias which reduces the magnitude of the PAC metric $\left(P L V_{P A C}\right)$ in presence of a harmonic PAC pattern. On the other hand, Figures B.4 and B.5 illustrate the complementary situation in which the magnitude of the PAC metric $\left(M V L_{P A C}\right)$ in absence of PAC is biased from closed to zero (see Figure B.5B) toward higher magnitude values $\left(\left|M V L_{P A C}\right| \approx 0.6\right.$ in Figure B.5B), as a consequence of the presence of phase clustering $\left(P C_{L F}\right)$. In Figures B.4 and B.5 is also shown that the presence phase clustering $\left(P C_{L F}\right)$ introduces a bias that reduces the magnitude of the harmonicity metric (TLI) in presence of harmonically related oscillations $\left(f_{0}=f_{L F}=3 \mathrm{~Hz}\right.$ and $f_{H F}=89 \times f_{L F}=267$ $\mathrm{Hz})$. 
bioRxiv preprint doi: https://doi.org/10.1101/2020.10.15.341800; this version posted November 1, 2020. The copyright holder for this preprint (which was not certified by peer review) is the author/funder, who has granted bioRxiv a license to display the preprint in perpetuity. It is made available under aCC-BY-NC-ND 4.0 International license.

A

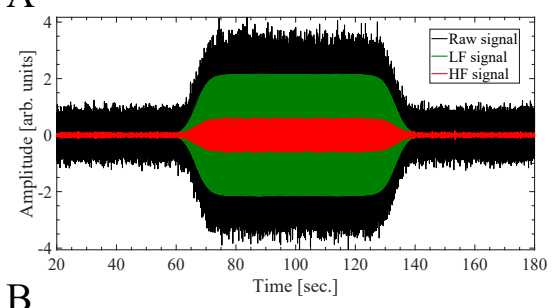

B
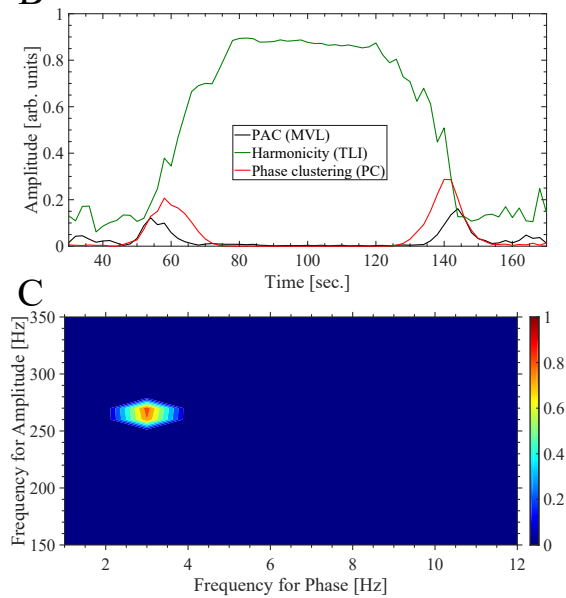

$\mathrm{D}$

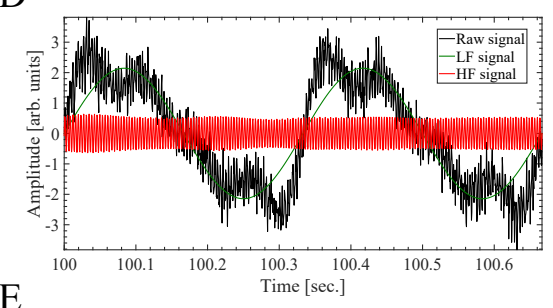

$\mathrm{E}$

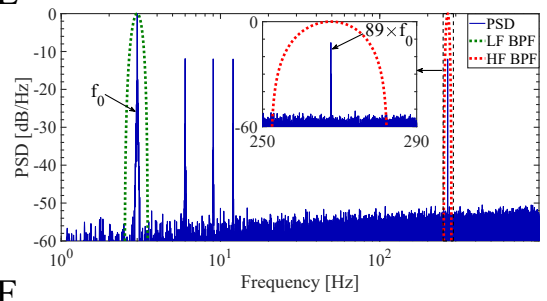

$\mathrm{F}$

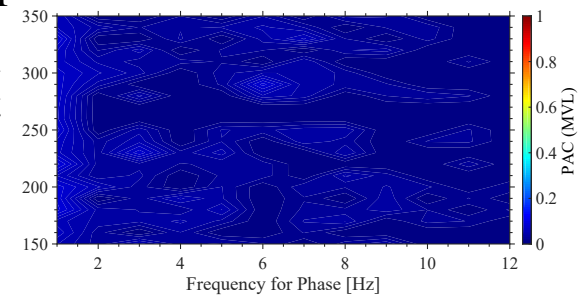

Figure B.4: Temporal evolution of the PAC $\left(M V L_{P A C}\right)$, harmonicity (TLI) and phase clustering $\left(P C_{L F}\right)$ metrics during a synthetic oscillatory dynamics constituted by harmonically related rhythms with no PAC. The synthetic dynamics was synthesized using the same parameter values than those used in Figure B.2 The $M V L_{P A C}$ (see Eq. 5, TLI and $P C_{L F}$ metrics were computed using the same set of band pass-filters and hyperparameter values than those used in Figure B.2 The description of the panels is the same than that given in Figure B.2 
bioRxiv preprint doi: https://doi.org/10.1101/2020.10.15.341800; this version posted November 1, 2020. The copyright holder for this preprint (which was not certified by peer review) is the author/funder, who has granted bioRxiv a license to display the preprint in perpetuity. It is made available under aCC-BY-NC-ND 4.0 International license.

A

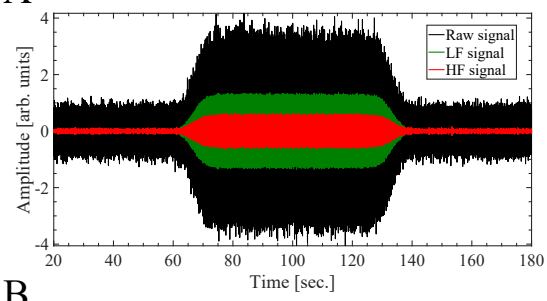

B
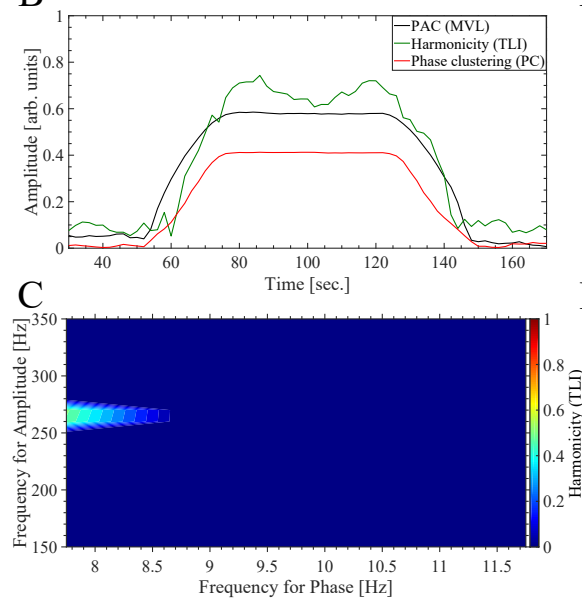

$\mathrm{D}$

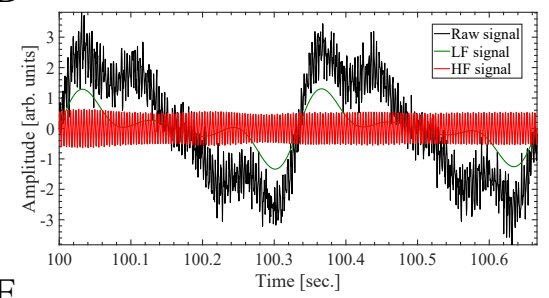

$\mathrm{E}$
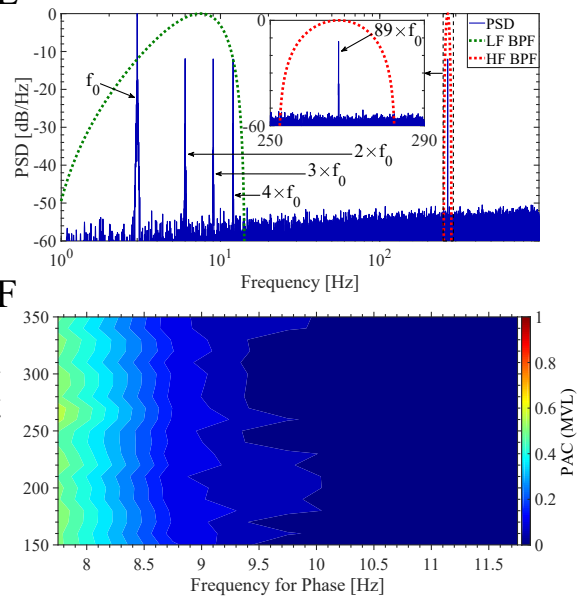

Figure B.5: Temporal evolution of the PAC $\left(M V L_{P A C}\right)$, harmonicity (TLI) and phase clustering $\left(P C_{L F}\right)$ metrics during a synthetic oscillatory dynamics constituted by harmonically related rhythms with no PAC. In this plot we use the same synthetic dynamics and the same set of hyperparameter values to compute the metrics than those described in the caption of Figure B.2 except for the bandwidth of the BPF used to compute the LF component $\left(B w_{L F}\right)$. In this case, the $M V L_{P A C}$ (see Eq. 5), TLI and $P C_{L F}$ metrics were computed using $B w_{L F}=13.5 \mathrm{~Hz}$ centered around $7.5 \mathrm{~Hz}$ (see the dotted green line in panel E). This wide BPF produces a non sinusoidal LF component (see solid green line in panel D), characterized by a non uniform distribution of phase values producing the increase of the phase clustering $\left(P C_{L F}\right)$ during the dynamics (see solid red line in panel B). Note the bias in the PAC $\left(M V L_{P A C}\right)$ and harmonicity (TLI) metrics due to the presence of phase clustering $\left(P C_{L F}\right)$. The description of the panels is the same than that given in Figure B.2

\section{Appendix B.3. A single oscillatory dynamics characterized by dependent fre- quencies}

Figure B.6 shows the phase portraits for the simulated dynamics of the Van der Pol oscillator, complementing the results shown in Figure 15 of the main text. 

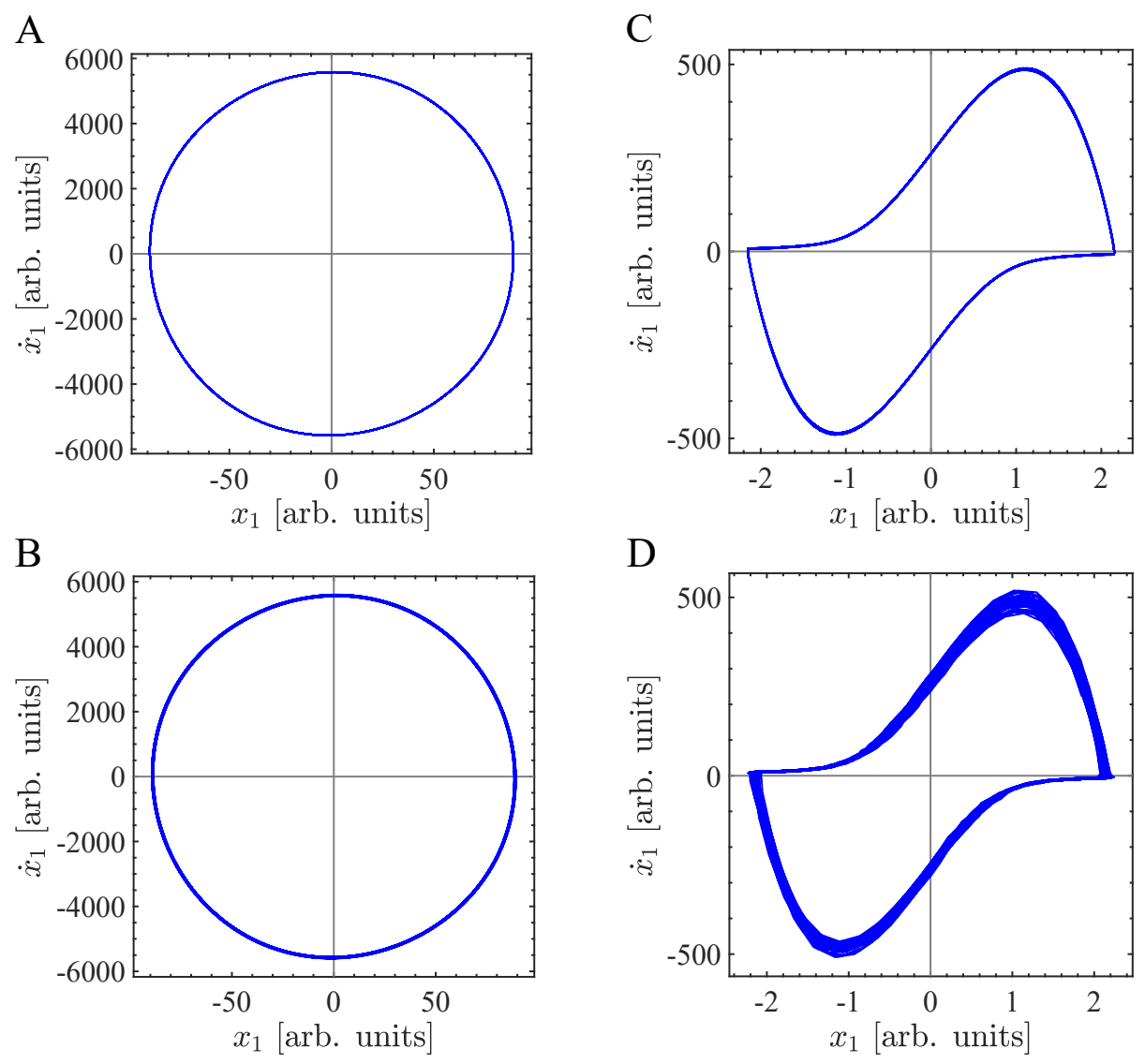

Figure B.6: Phase portraits for the simulated dynamics of the Van der Pol oscillator. In this figure we use the same synthetic dynamics and the same set of hyperparameter values than those described in the caption of Figure 15 In particular, the phase portraits were computed using the dynamics $x_{1}$ in Eq. A.14 which only takes into account the effect of the intrinsic noise, that is, without including the extrinsic (i.e. of observation) noise $\eta$. (A) Phase portrait corresponding to the dynamics shown in Figure $15 \mathrm{~A}$ (No PAC). (B) Phase portrait corresponding to the dynamics shown in Figure 15F (No PAC). (C) Phase portrait corresponding to the dynamics shown in Figure 15D (Harmonic PAC). (D) Phase portrait corresponding to the dynamics shown in Figure $15 \mathrm{~K}$ (Non harmonic PAC).

Figure B.7 shows the harmonicity-PAC plot usnig the TLI, $P L V_{P A C}$ and $K L M I_{P A C}$ metrics computed for the simulated dynamics of the Van der Pol oscillator with intrinsic noise of type non-additive white Gaussian noise (NAWGN). 

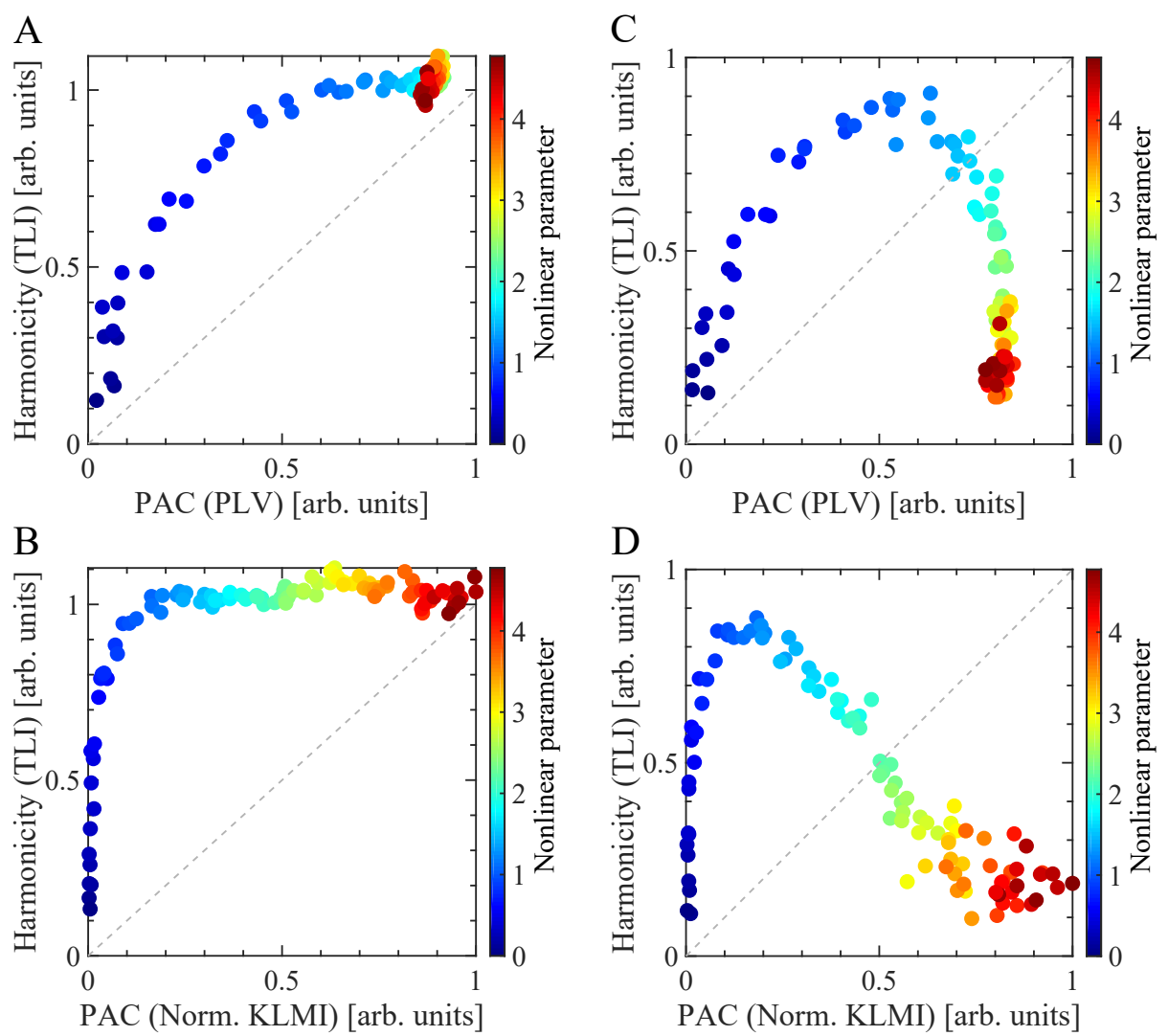

Figure B.7: Harmonicity-PAC plot computed for the simulated dynamics of the Van der Pol oscillator with intrinsic noise of type non-additive white Gaussian noise (NAWGN). In this figure we use the same synthetic dynamics and the same set of hyperparameter values to compute the metrics than those described in the caption of Figure 15 except for the configuration of the intrinsic noise. In this case, we use non-additive white Gaussian noise (NAWGN). For the numerical integration of the stochastic differential equation A.14 we use an explicit solver based on the Euler-Heun method 41] using the Stratonovich integral formulation. Importantly, we verified that the harmonicity-PAC plots shown in this figure do not change when computed using the Itô integral formulation. For the panels A and B, the dynamics of the Van der Pol oscillator was simulated using intrinsic noise of type NAWGN applied only on the equation of $\dot{x}_{2}\left(g_{1}=0\right.$ and $g_{2}=0.5 x_{2}$ in Eq. A.14). For the panels C and D, the dynamics was simulated by applying the intrinsic noise of type non-additive white Gaussian noise (NAWGN) on the equations of both $\dot{x}_{1}$ and $\dot{x}_{2}$ (i.e. $g_{1}=0.5 x_{1}$ and $g_{2}=0.5 x_{2}$ in Eq. A.14. Therefore, in this case the intrinsic noise components (NAWGN) in Eq. A.14 result $\eta_{1} \approx \mathcal{N}\left(0,0.5 x_{1}\right)$ and $\eta_{2} \approx \mathcal{N}\left(0,0.5 x_{2}\right)$. Extrinsic noise $\eta(t)$ was added as shown in Eq. A.14 In this case the noise level corresponds to the 10 percent of the maximum amplitude of the dynamics $x_{1}$ in Eq. A.14, scaling the standard deviation $\sigma$ of the additive white Gaussian noise (AWGN) $\eta \approx \mathcal{N}(0, \sigma)$. The harmonicity metric (TLI) was computed as it was described in Section 2.4 For the panels $\mathrm{A}$ and $\mathrm{C}$, the PAC metric $\left(P L V_{P A C}\right)$ was computed using Eq. 4 with the configuration given by Eq. A.20 and $M=N=1$. For the panels B and D, we compute the $K L M I_{P A C}$ using Eqs. 6 and 7 with the configuration given by Eq. A.20 Note that the $K L M I_{P A C}$ was normalized with its maximum value in each plot.

Figure B.8 shows the phase portraits for the simulated dynamics of the Van 


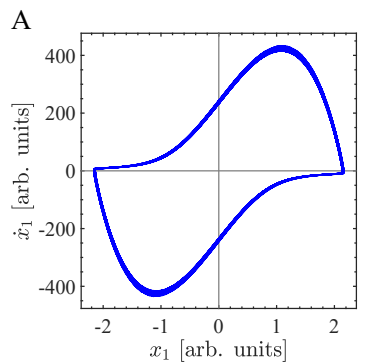

B

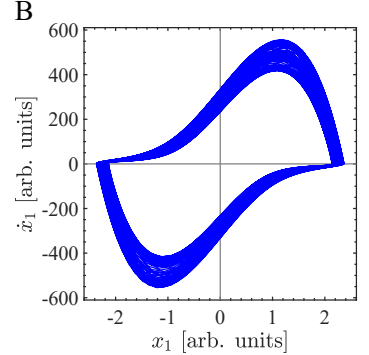

$\mathrm{C}$

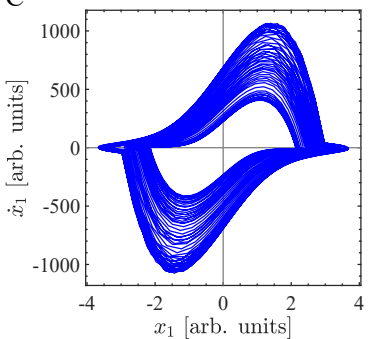

Figure B.8: Phase portraits illustrating the simulated dynamics of the Van der Pol oscillator under an amplitude-modulated external driving. In this figure we use the same synthetic dynamics and the same set of hyperparameter values than those used to compute Figure $16 \mathrm{C}$. In particular, the phase portraits were computed using the dynamics $x_{1}$ in Eq. A.14 which only takes into account the effect of the intrinsic noise, that is, without including the extrinsic (i.e. of observation) noise $\eta$. (A) Phase portrait corresponding to the dynamics shown in Figure $16 \mathrm{C}$ for $A_{e} /\left(5 \times 10^{4}\right) \approx 0.01$. (B) Phase portrait corresponding to the dynamics shown in Figure $16 \mathrm{C}$ for $A_{e} /\left(5 \times 10^{4}\right) \approx 0.1$. (C) Phase portrait corresponding to the dynamics shown in Figure $16 \mathrm{C}$ for $A_{e} /\left(5 \times 10^{4}\right) \approx 1$.

Appendix B.4. Two coupled oscillatory dynamics characterized by independent frequencies

Figure B.9 shows the phase portraits for the simulated dynamics of the 2nd order parametric oscillator, complementing the results shown in Figure 17 of the main text. 


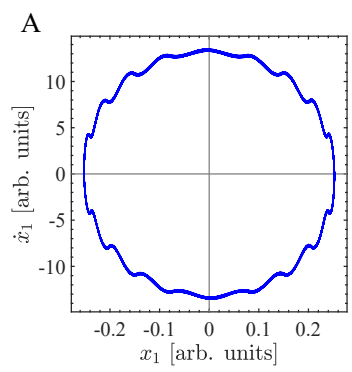

B

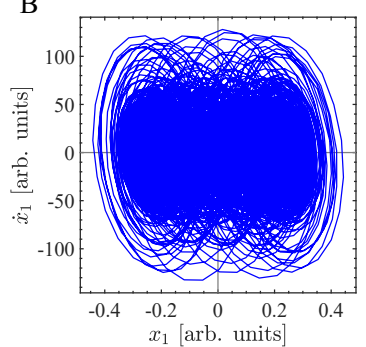

C

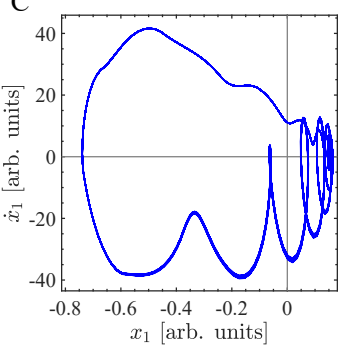

$\mathrm{D}$

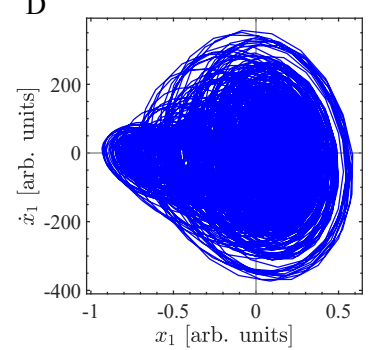

E

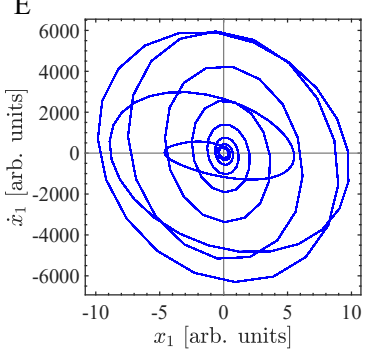

$\mathrm{F}$

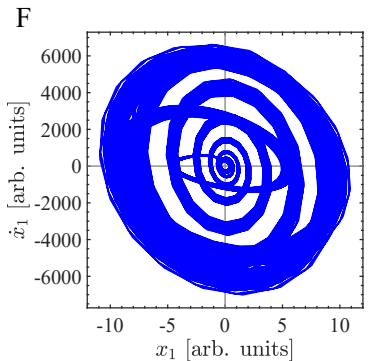

Figure B.9: Phase portraits for the simulated dynamics of the 2nd order parametric oscillator. In this figure we use the same synthetic dynamics and the same set of hyperparameter values than those described in the caption of Figure 17 In particular, the phase portraits were computed using the dynamics $x_{1}$ in Eq. A.18 which only takes into account the effect of the intrinsic noise, that is, without including the extrinsic (i.e. of observation) noise $\eta$. (A) Phase portrait corresponding to the dynamics shown in Figure $17 \mathrm{~A}, \mathrm{~B}$ (No PFC). (B) Phase portrait corresponding to the dynamics shown in Figure 17 H,I (No PFC). (C) Phase portrait corresponding to an intermediate dynamics in between the cases shown in Figure 17A,B and Figure 17D,E. (D) Phase portrait corresponding to the dynamics shown in Figure $17 \mathrm{~K}, \mathrm{~L}$ (Non harmonic PFC). (E) Phase portrait corresponding to the dynamics shown in Figure 17P,E (Harmonic PFC). (F) Phase portrait corresponding to the dynamics shown in Figure 17F, G (Harmonic PFC).

Figures B.10 and B.11 show the PFC patterns corresponding to the oscillator dynamics generated by simultaneously applying an off-resonance external driving $F_{e}$ with the parametric driving $W_{p}$ tuned at the same frequency $f_{e}=f_{p}=f_{0} / 11.62 \approx 8.61 \mathrm{~Hz}$ and $\theta_{e}=\pi / 2$ (see Eqs. A.16 and A.17). For the configuration used to compute the Figures B.10J and B.11], the intrinsic noise is capable to drive the resonator at its natural frequency $f_{0}$ for low $A_{p}$ values (see panels $\mathrm{H}$ and I in Figures B.10 and B.11). However, no harmonicity is observed in Figures B.10J and B.11J for low $A_{p}$ values (see blue filled circles in Figures B.10J and B.11 J), due to the fact that we configured the external $\left(f_{e}\right)$ and parametric $\left(f_{p}\right)$ driving frequencies having a non harmonic ratio with the natural resonance frequency $\left(f_{0}\right)$ of the undamped oscillator $(\mu=0)$, i.e. $f_{e}=f_{p}=f_{0} / 11.62 \approx 8.6 \mathrm{~Hz}$. In this regard, compare the harmonicity for low $A_{p}$ values (blue filled circles) in Figures [17J, B.10] and B.11J. 

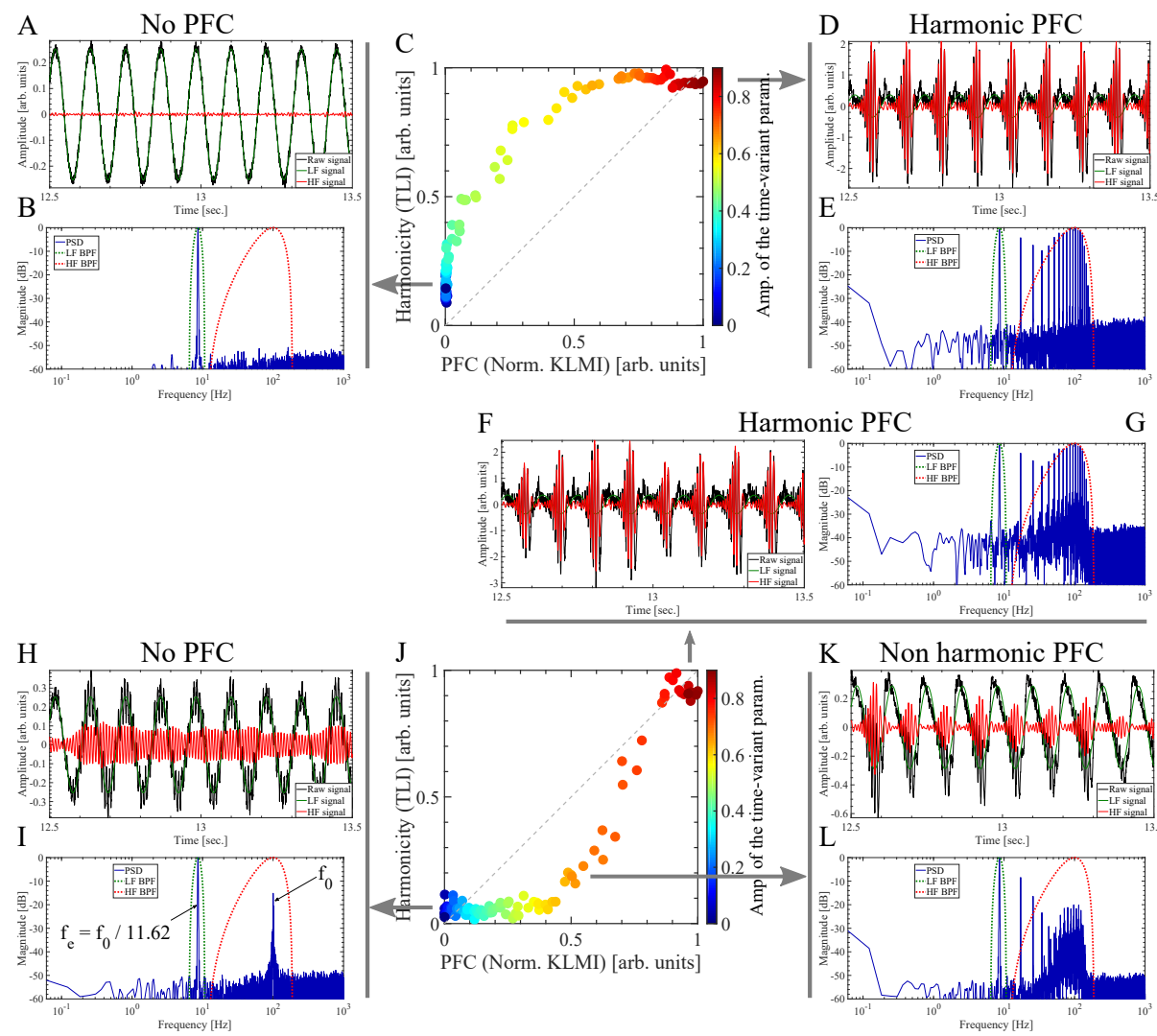

Figure B.10: Harmonicity-PFC plot computed for the simulated dynamics of the 2nd order parametric oscillator with intrinsic noise of type additive white Gaussian noise (AWGN). Note that two oscillatory dynamics with independent frequencies can produce harmonic PFC patterns (panels D, E and F, G). In this figure we use the same synthetic dynamics and the same set of hyperparameter values to compute the metrics than those described in the caption of Figure 17 except for the phase of the external driving $\theta_{e}=\pi / 2$ and the frequency of the parametric and external driving, which were configured as $f_{p}=f_{e}=f_{0} / 11.62 \approx 8.3 \mathrm{~Hz}$ (i.e. $f_{p}$ and $f_{e}$ are non harmonics of $f_{0}$ ). The harmonicity metric (TLI) was computed as it was described in Section 2.4 The PFC metric $\left(K L M I_{P F C}\right)$ was computed using Eqs. 6 and 7 with the configuration given by Eq. A.22 Note that the $K L M I_{P F C}$ was normalized with its maximum value in each plot. 

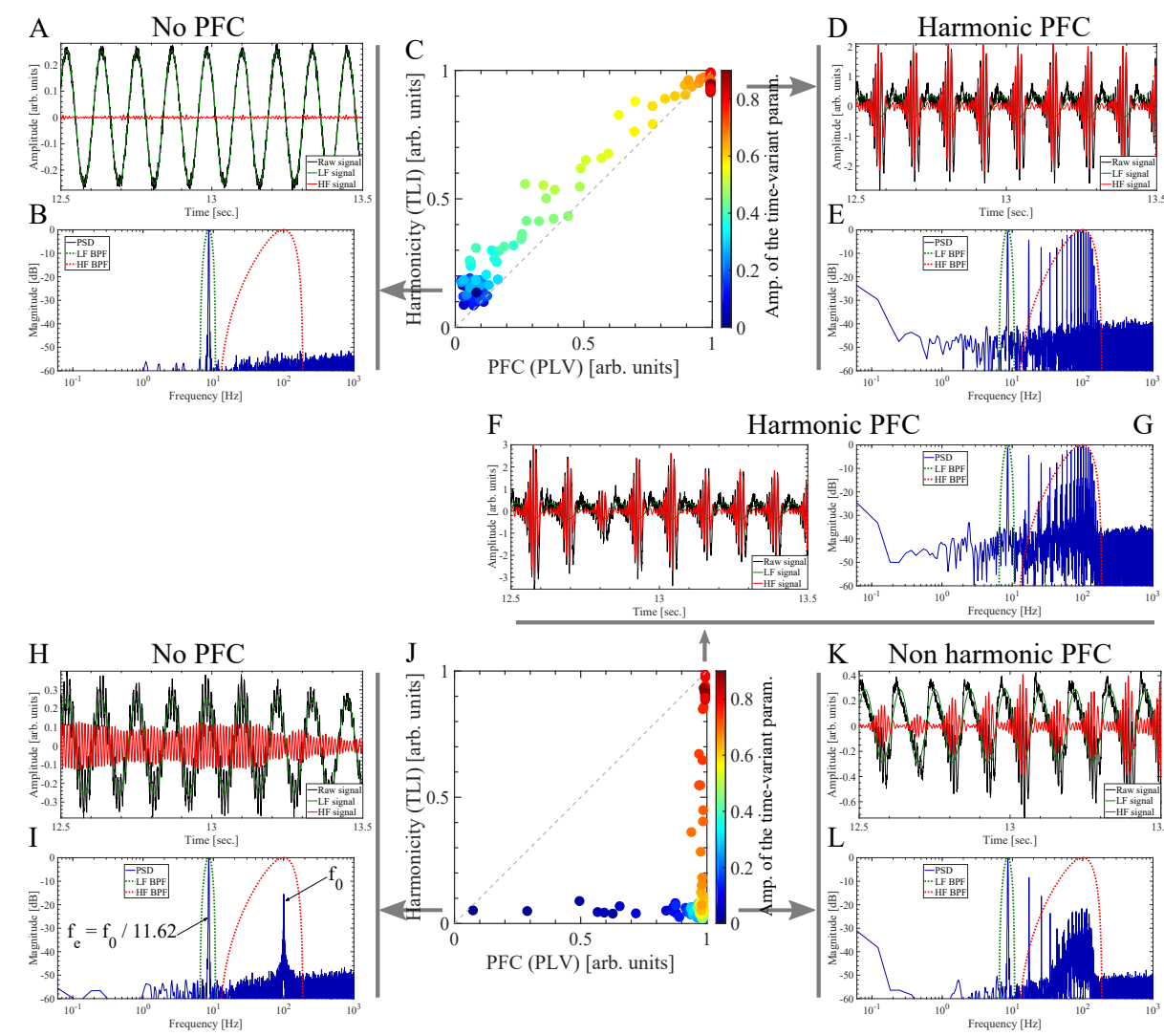

Harmonic PFC

Figure B.11: Harmonicity-PFC plot computed for the simulated dynamics of the 2nd order parametric oscillator with intrinsic noise of type additive white Gaussian noise (AWGN). Note that two oscillatory dynamics with independent frequencies can produce harmonic PFC patterns (panels D, E and F, G). In this figure we use the same synthetic dynamics and the same set of hyperparameter values to compute the filtering and harmonicity metric (TLI) than those described in the caption of Figure 17 except for the phase of the external driving $\theta_{e}=\pi / 2$ and the frequency of the parametric and external driving, which were configured as $f_{p}=f_{e}=f_{0} / 11.62 \approx 8.3 \mathrm{~Hz}$ (i.e. $f_{p}$ and $f_{e}$ are non harmonics of $f_{0}$ ). The harmonicity metric (TLI) was computed as it was described in Section 2.4 In this case, the PFC metric $\left(P L V_{P F C}\right)$ was computed using Eq. 4 with the configuration given by Eq. A.22 and $M=1$, $N=1$.

\section{Appendix B.5. Biologically plausible neural network model}

Figure B.12 shows the harmonicity-PAC plots computed for the for the simulated dynamics of the biologically plausible neural network model shown in Figure 1 using the softplus activation function $S\left(I_{i}\right)$ (Eq. 3). The results shown in Figure B.12 should be compared with those shown in Figure 18 of the main text. 
bioRxiv preprint doi: https://doi.org/10.1101/2020.10.15.341800; this version posted November 1, 2020. The copyright holder for this preprint (which was not certified by peer review) is the author/funder, who has granted bioRxiv a license to display the preprint in perpetuity. It is made available under aCC-BY-NC-ND 4.0 International license.
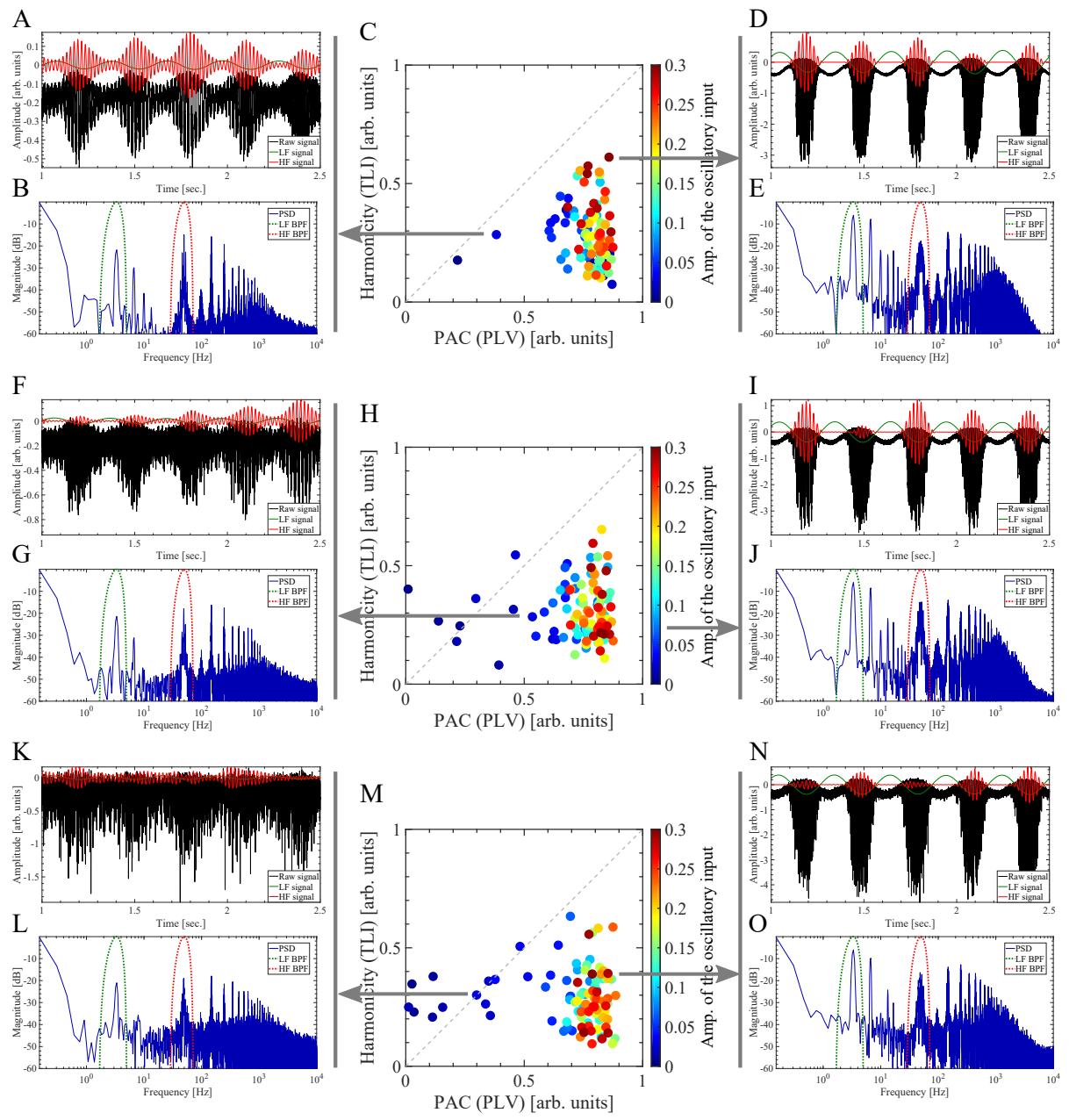

Figure B.12: Harmonicity-PAC plot computed for the simulated dynamics of the biologically plausible neural network model shown in Figure 1 using the softplus activation function $S\left(I_{i}\right)$ (Eq. 3). In this figure we use the same synthetic dynamics and the same set of hyperparameter values to compute the metrics than those described in the caption of Figure 18 except for the activation function $S\left(I_{i}\right)$ which in this case was computed using the Eq. 33 with $c=20$. 